\title{
A curvilinear high order finite element framework for electromechanics: from linearised electro-elasticity to massively deformable dielectric elastomers
}

\author{
Roman Poya ${ }^{a, b 1}$, Antonio J. Gil ${ }^{a 2}$, Rogelio Ortigosa ${ }^{a}$, Ruben Sevilla ${ }^{a}$, Javier Bonet ${ }^{c}$, Wolfgang \\ A. Wall ${ }^{b}$ \\ ${ }^{a}$ Zienkiewicz Centre for Computational Engineering, College of Engineering, Swansea University, Bay Campus, \\ SA1 8EN, United Kingdom \\ ${ }^{b}$ Institute for Computational Mechanics, Technische Universität München, Boltzmannstrasse 15, D-85748 \\ Garching b. München, Germany \\ ${ }^{c}$ University of Greenwich, London, SE10 9LS, United Kingdom
}

\begin{abstract}
This paper presents a high order finite element implementation of the convex multi-variable electro-elasticity for large deformations large electric fields analyses and its particularisation to the case of small strains through a staggered scheme. With an emphasis on accurate geometrical representation, a high performance curvilinear finite element framework based on an a posteriori mesh deformation technique is developed to accurately discretise the underlying displacementpotential variational formulation. The performance of the method under near incompressibility and bending actuation scenarios is analysed with extremely thin and highly stretched components and compared to the performance of mixed variational principles recently reported by Gil and Ortigosa $[1,2,3]$. Although convex multi-variable constitutive models are elliptic hence, materially stable for the entire range of deformations and electric fields, other forms of physical instabilities are not precluded in these models. In particular, physical instabilities present in dielectric elastomers such as pull-in instability, snap-through and the formation, propagation and nucleation of wrinkles and folds are numerically studied with a detailed precision in this paper, verifying experimental findings. For the case of small strains, the essence of the approach taken lies in guaranteeing the objectivity of the resulting work conjugates, by starting from the underlying convex multi-variable internal energy, whence avoiding the need for further symmetrisation of the resulting Maxwell and Minkowski-type stresses at small strain regime. In this context, the nonlinearity with respect to electrostatic counterparts such as electric displacements is still retained, hence resulting in a formulation similar but more competitive with the existing linearised electro-elasticity approaches. Virtual prototyping of many application-oriented dielectric elastomers are carried out with an eye on pattern forming in soft robotics and other potential medical applications.
\end{abstract}

Keywords: Monolithic \& staggered electro-elasticity, high order curvilinear meshes, dielectric elastomers, material instability, wrinkling

\footnotetext{
${ }^{1}$ Corresponding author: r.poya@swansea.ac.uk

${ }^{2}$ Corresponding author: a.j.gil@swansea.ac.uk
} 


\section{Introduction}

In recent years, exploiting actuation and harvesting through the heterogenous class of ElectroActive-Polymers (EAP) has received considerable research focus. In particular, the electronic subgroup of EAP such as Dielectric Elastomers (DE) and electrostrictive relaxor ferroelectric polymers or Piezoelectric Polymers (PP) have become the subject of intensive mathematical and numerical analyses. Typically, the physically insightful information that could be gained from the numerical studies, depends on the capability of the underlying mathematical model utilised to simulate EAP. This has led to the development of a diverse range of mathematical models ranging from simplified to high fidelity models $[4,5]$.

On one end of the spectrum lies the class of simplified formulations, where one-dimensional idealisation in the form of rod and beam structures with mass-spring-damper-capacitor support using small strains and linear electrostatic assumption is utilised [4, 6, 7]. Particularly more popular in the experimental physics community, these models have been used successfully to characterise actuation and energy harvesting capabilities of a range of materials, inherently due to their proximity to the actual experimental set-up. The potential of a material, in exhibiting electrostiction is typically exploited using such simplified formulations [8, 9, 10, 11, 12, 13].

On the other end of the spectrum lies the class of mathematically more sophisticated formulations that exploit the large deformation characteristics of EAP $[5,14,15,1,2,16,17,18]$. The point of departure for such formulations is an assumed energy functional for the coupled electromechanical system. Conceptually, essential and suitable mathematical requirements for the energy functional such as ellipticity [19, 20], multi-variable convexity [1, 2], coercivity [21] and material frame indifference [22] can only be studied in a large deformation context. From a phenomenological point of view, these requirements or rather restrictions have important physical implications, in particular in guaranteeing the positive definiteness of the generalised electromechanical acoustic tensor, existence of real wave speeds in the material in the vicinity of an equilibrium configuration and the electromechanical stability of the material [23, 2]. Apart from these requirements other forms of physical instabilities present in dielectric elastomers such as pull-in instability, snap-through and the formation, propagation and nucleation of wrinkles have also been reported for these models, numerically as well experimentally [24, 25, 26, 27, 28, 29, 30, 31].

In an important intermediate class for electromechanics, the large deformation characteristics of the system are neglected, whereas the nonlinearity still present in the material emanates from the electrostriction of the material through the Maxwell (for vacuum $V_{\infty}$ ) or Minkowski (for material $V$ ) stress tensors $[32,33,34,35]$. Theoretical aspects of these formulations were first introduced in Landau and Lifshitz [33]. The practical relevance of Maxwell stress tensor has led to a widespread utilisation of these formulations for exploiting electrostriction and magnetostriction. Unfortunately, electrostrictive models based on the utilisation of Minkowski stress tensor, in the generic case of anisotropy do not satisfy material frame indifference (i.e. objectivity or invariance of the energy with respect to rotations) of the electromechanical (total) stress tensor, due to the inherent non-symmetric nature of the Minkowski stress. Several authors in the past have used ad-hoc solutions, such as symmetrisation of the total stress tensor, or consideration of the conservation of angular momentum in the formulation, as a remedy $[34,36]$. Nevertheless, the extended electromechanical Hessian still remains non-symmetric, which dictates the development of specialised non-symmetric finite element frameworks. Recently, Bustamente [37] has shown that physically admissible energy functionals can be constructed by choosing suitable constitutive restrictions such that their linearisation yields objective Minkowski-type stresses. 
The present manuscript presents a computational framework suitable for both geometrically linearised and large deformation large electric field electromechanics. A convex multi-variable strain energy description based on the works of Gil and Ortigosa $[1,2,3]$ is chosen for modelling EAPs under actuation and energy harvesting scenarios. For the case of small strains, following Bustamente [37], the present manuscript extends the framework developed by Gil and Ortigosa $[1,2]$ to the case of geometrically linearised electrostriction, to redress the aforementioned inconsistencies for the class of intermediate formulations. Importantly, all the aforementioned mathematical requirements are imposed at a large deformation level to arrive at a physically admissible energy functional. In this context, convex multi-variable energies typically expressed in terms of fundamental kinematic measures $\{\boldsymbol{F}, \boldsymbol{H}, J\}$ are re-expressed in terms of a set of symmetric kinematics $\{\boldsymbol{C}, \boldsymbol{G}, C\}$ to guarantee the objectivity of the energy functional. Linearisation with respect to geometrical fields is then performed by perturbing the energy in the vicinity of the reference configuration. Analogous to [38], this is achieved through a staggered scheme where the equations of electrostatics are solved for in a nonlinear fashion whereas the linearised mechanical equations are updated incrementally.

Admittedly, all the three class of formulations have been primarily applied to simplified geometries where the aim has been to verify the computational framework rather than to simulate realistic electromechanical components. In the present work, emphasis is put on accurate geometrical representation of electromechanical components by utilising an a posteriori curvilinear mesh deformation technique to accurately represent the true CAD boundaries. This is achieved by placing the high order nodes in the computational mesh on the true CAD boundary (curves/surfaces) through the solid mechanics based mesh deformation technique [38], but the underlying finite element functional spaces are not modified, i.e. a standard isoparametric finite element discretisation is employed. By relying on accurate geometrical representation and standard finite element technology, the current framework takes finite element analysis of electromechanical systems beyond the verification stage through virtual prototyping of a series of electromechanical components with potential applications in pattern forming, soft-robotics and other medical applications.

The paper is organised as follows. In section 2, nonlinear continuum electromechanics is described. In section 3, a variational framework for displacement-potential electromechanics is described. Aspects of particularisation to small strains and the corresponding staggered scheme are also discussed in this section. In section 4, a series of numerical examples pertaining to the capability of the current framework in modelling DEs are analysed, starting from the $h$ and $p$ convergence properties of the curvilinear finite element framework presented in subsection 4.1. The effect of accurate boundary representation using high order curvilinear finite elements is analysed in subsection 4.2 and compared to high order planar elements (elements with planar faces/edges). In subsection 4.3, examples of electromechanical actuation for linearised electromechanics using the presented staggered scheme are discussed. In subsection 4.4, the performance of the current high order finite element displacement-potential approach is compared to those of mixed $\mathrm{Hu}$-Washizu formulations presented in $[1,2,3]$. In subsection 4.5, impact of code transformation and data parallelism for an efficient implementation of convex multi-variable electro-elasticity in the context of high order finite elements is presented. Finally, a series of examples pertaining to the massive deformation of dielectric elastomers are presented in subsection 4.6. The inherent instabilities in DEs such as pull-in instability and the formation of wrinkles are studied with detailed precision using $h$ and $p$ refinements, pinpointing the robustness and the high performant 
capability of the current framework.

\section{Nonlinear continuum electromechanics}

\subsection{Kinematics: motion and deformation}

Let us consider the motion of an electro-active body which in its initial or material configuration is defined by a domain $V$ of boundary $\partial V$ with outward unit normal $\boldsymbol{N}$. After the motion, the body occupies a spatial configuration defined by a domain $v$ of boundary $\partial v$ with outward unit normal $\boldsymbol{n}$, as shown in Figure 1. The motion of the electro-active polymer $V$ is defined by a pseudo-time $(t)$ dependent mapping field $\boldsymbol{\phi}$ which links a material particle from material configuration $\boldsymbol{X}$ to spatial configuration $\boldsymbol{x}$ according to $\boldsymbol{x}=\boldsymbol{\phi}(\boldsymbol{X}, t)$, where displacement boundary conditions can be defined as $\boldsymbol{x}=(\boldsymbol{\phi})_{\partial_{u} V}$ on the boundary $\partial_{u} V \subset \partial V$, where the notation $(\bullet)_{\partial_{u} V}$ is used to indicate the given value of a variable $(\bullet)$ on the boundary $\partial_{u} V$. The two-point

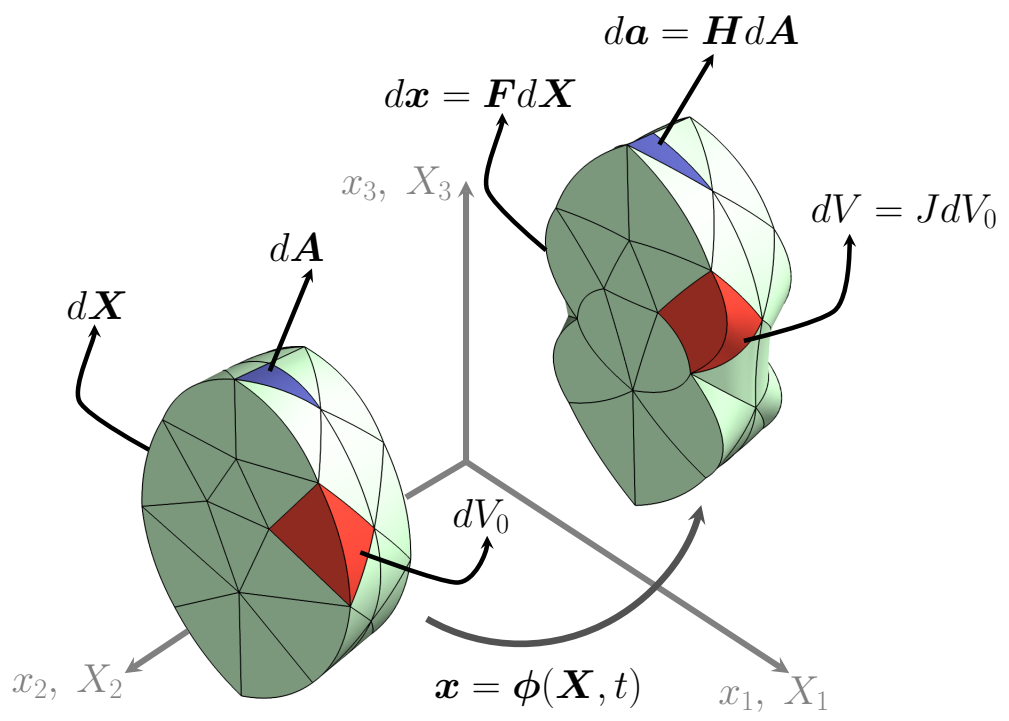

Figure 1: Deformation map of a continuum and illustration of the strain measures $\boldsymbol{F}, \boldsymbol{H}$ and $J$.

deformation gradient tensor or fibre-map $\boldsymbol{F}$, which relates a fibre of differential length from the material configuration $d \boldsymbol{X}$ to the spatial configuration $d \boldsymbol{x}$, namely $d \boldsymbol{x}=\boldsymbol{F} d \boldsymbol{X}$, is defined as [39]

$$
\boldsymbol{F}=\nabla_{0} \boldsymbol{x}=\frac{\partial \phi(\boldsymbol{X}, t)}{\partial \boldsymbol{X}}
$$

where $\boldsymbol{\nabla}_{0}(\bullet)$ is the material or Lagrangian gradient operator. In addition, $J=\operatorname{det} \boldsymbol{F}$ represents the Jacobian or volume-map of the deformation, which relates differential volume elements in the material configuration $d V$ and the spatial configuration $d v$ as $d v=J d V$. Finally, the element area vector is mapped from initial $d \boldsymbol{A}$ (colinear with $\boldsymbol{N}$ ) to final $d \boldsymbol{a}$ (colinear with $\boldsymbol{n}$ ) configuration by means of the two-point co-factor or adjoint tensor $\boldsymbol{H}$ as $d \boldsymbol{a}=\boldsymbol{H} d \boldsymbol{A}$, which is related to the deformation gradient via the so-called Nanson's rule as $\boldsymbol{H}=J \boldsymbol{F}^{-T}$. With the help of the definition of the tensor cross product presented in [40] and utilised in the context of hyperelasticity 
and electromechanics in $[41,22,1]$, it is possible to re-write the area $\boldsymbol{H}$ and volume $J$ maps as

$$
\boldsymbol{H}=\frac{1}{2} \boldsymbol{F} \times \boldsymbol{F} ; \quad J=\frac{1}{3} \boldsymbol{H}: \boldsymbol{F},
$$

where for two-point second order tensors $\boldsymbol{A}$ and $\boldsymbol{B}$, the tensor cross operation is computed as $[\boldsymbol{A} \times \boldsymbol{B}]_{i I}=\mathcal{E}_{i j k} \mathcal{E}_{I J K} A_{j J} B_{k K}$, with $\mathcal{E}$ the third order alternating tensor ${ }^{3}$. Based on the fundamental kinematic measures $\{\boldsymbol{F}, \boldsymbol{H}, J\}$ defined in (1) and (2), a set of symmetric kinematic measures namely $\boldsymbol{C}, \boldsymbol{G}$ and $C$, can be defined as follows [22]

$$
\boldsymbol{C}=\boldsymbol{F}^{T} \boldsymbol{F} ; \quad \boldsymbol{G}=\frac{1}{2} \boldsymbol{C} \times \boldsymbol{C}=\boldsymbol{H}^{T} \boldsymbol{H} ; \quad C=\frac{1}{3} \boldsymbol{G}: \boldsymbol{C}=J^{2},
$$

where $\boldsymbol{C}$ is the right Cauchy-Green strain tensor and $\boldsymbol{G}$ and $C$ are its co-factor and determinant, respectively. Let us define $\delta \boldsymbol{u}$ and $\Delta \boldsymbol{u}$ as virtual and incremental variations of $\boldsymbol{x}$, respectively, where it will be assumed that $\delta \boldsymbol{u}$ and $\Delta \boldsymbol{u}$ satisfy compatible homogeneous displacement based boundary conditions that vanish on $\partial_{u} V$. Making use of the tensor cross product operation introduced above, the first and second directional derivatives of the symmetric kinematic measures $\{\boldsymbol{C}, \boldsymbol{G}, C\}$ with respect to virtual and incremental variation of the geometry can now be evaluated, starting with $\boldsymbol{C}$

$$
\begin{aligned}
D \boldsymbol{C}[\delta \boldsymbol{u}] & =\left(\boldsymbol{\nabla}_{0} \delta \boldsymbol{u}\right)^{T} \boldsymbol{F}+\boldsymbol{F}^{T} \boldsymbol{\nabla}_{0} \delta \boldsymbol{u} \\
D^{2} \boldsymbol{C}[\delta \boldsymbol{u} ; \Delta \boldsymbol{u}] & =\left(\boldsymbol{\nabla}_{0} \delta \boldsymbol{u}\right)^{T}\left(\boldsymbol{\nabla}_{0} \Delta \boldsymbol{u}\right)+\left(\boldsymbol{\nabla}_{0} \Delta \boldsymbol{u}\right)^{T}\left(\boldsymbol{\nabla}_{0} \delta \boldsymbol{u}\right)
\end{aligned}
$$

and similarly for the co-factor $\boldsymbol{G}$

$$
\begin{aligned}
D \boldsymbol{G}[\delta \boldsymbol{u}] & =\boldsymbol{C} \times D \boldsymbol{C}[\delta \boldsymbol{u}] \\
D^{2} \boldsymbol{G}[\delta \boldsymbol{u} ; \Delta \boldsymbol{u}] & =\boldsymbol{C} \times D^{2} \boldsymbol{C}[\delta \boldsymbol{u} ; \Delta \boldsymbol{u}]+D \boldsymbol{C}[\Delta \boldsymbol{u}] \times D \boldsymbol{C}[\delta \boldsymbol{u}]
\end{aligned}
$$

and finally for the determinant $C$

$$
\begin{aligned}
D C[\delta \boldsymbol{u}] & =\boldsymbol{G}: D \boldsymbol{C}[\delta \boldsymbol{u}] \\
D^{2} C[\delta \boldsymbol{u}, \Delta \boldsymbol{u}] & =D^{2} \boldsymbol{C}[\delta \boldsymbol{u} ; \Delta \boldsymbol{u}]: \boldsymbol{G}+\boldsymbol{C}:(D \boldsymbol{C}[\delta \boldsymbol{u}] \times D \boldsymbol{C}[\Delta \boldsymbol{u}])
\end{aligned}
$$

\subsection{Translational and rotational equilibruim}

The kinematics of the continuum presented in subsection 2.1 must be described through the conservation of linear momentum. In the absence of inertial effects, the global conservation of linear momentum leads to the integral translational equilibrium equations

$$
\int_{\partial_{t} V} \boldsymbol{t}_{0} d A+\int_{V} \boldsymbol{f}_{0} d V=\mathbf{0},
$$

\footnotetext{
${ }^{3}$ In addition, throughout the paper, the symbol $(\cdot)$ is used to indicate the scalar product or contraction of a single index $\boldsymbol{a} \cdot \boldsymbol{b}=a_{i} b_{i}$; the symbol (:) is used to indicate double contraction of two indices $\boldsymbol{A}: \boldsymbol{B}=A_{i j} B_{i j}$; the $\operatorname{symbol}(\times)$ is used to indicate the cross product between vectors $[\boldsymbol{a} \times \boldsymbol{b}]_{i}=\xi_{i j k} a_{j} b_{k}$ and the symbol $(\otimes)$ is used to indicate the outer or dyadic product $[\boldsymbol{a} \otimes \boldsymbol{b}]_{i j}=a_{i} b_{j}$.
} 
where $\boldsymbol{f}_{0}$ represents the body force per unit undeformed volume $V$ and $\boldsymbol{t}_{0}$ the traction force per unit undeformed area, applied on $\partial_{t} V \subset \partial V$ such that $\partial_{t} V \cup \partial_{u} V=\partial V$ and $\partial_{t} V \cap \partial_{u} V=\emptyset$. From $(7)$, the local translational equilibrium equations and the associated boundary conditions can be written as

$$
\begin{aligned}
\mathrm{DIVP}+\boldsymbol{f}_{0} & =\mathbf{0} & & \text { in } V ; \\
\boldsymbol{P} \boldsymbol{N} & =\boldsymbol{t}_{0} & & \text { on } \partial_{t} V ; \\
\phi & =(\phi)_{\partial_{\phi}} V & & \text { on } \partial_{u} V
\end{aligned}
$$

where $\boldsymbol{P}$ represents the first Piola-Kirchoff stress tensor. Furthermore, conservation of rotational equilibrium leads to the well-known tensor condition $\boldsymbol{P} \boldsymbol{F}^{T}=\boldsymbol{F} \boldsymbol{P}^{T}$.

\subsection{Electrostatics: Gauss's and Faraday's laws}

In addition to the conservation of translation and rotational equilibrium presented in subsection 2.2, the electro-active polymer represented by the continuum described in subsection 2.1 is subjected in its material configuration $V$ to an electric volume charge $\rho_{0}^{e}$ per unit of undeformed volume and an electric surface charge $\omega_{0}^{e}$ per unit of undeformed area applied on $\partial_{\omega} V \subset \partial V$. Hence, the integral version of the Gauss's law can be written in a Lagrangian format as

$$
\int_{\partial_{\omega} V} \omega_{0}^{e} d A+\int_{V} \rho_{0}^{e} d V=0
$$

From (9), the local version of Gauss's law and the associated boundary conditions can be written as

$$
\begin{aligned}
\operatorname{DIV} \boldsymbol{D}_{0}-\rho_{0}^{e} & =0 & & \text { in } V \\
\boldsymbol{D}_{0} \cdot \boldsymbol{N} & =-\omega_{0}^{e} & & \text { on } \partial_{\omega} V,
\end{aligned}
$$

where $\boldsymbol{D}_{0}$ is the Lagrangian electric displacement vector. Alternatively, the spatial electric displacement vector $\boldsymbol{D}$ can be obtained through the area push forward relationship $\boldsymbol{D}_{0}=\boldsymbol{H}^{T} \boldsymbol{D}$, $[5,14]$. Furthermore, in the absence of magnetic fields, the integral version of the static Faraday's law can be written in a Lagrangian form for a closed curve $\mathcal{C}$ embedded in $V \cup \partial V$ as

$$
\oint_{\mathcal{C}} \boldsymbol{E}_{0} \cdot d \boldsymbol{X}=0
$$

where $\boldsymbol{E}_{0}$ is the Lagrangian or material electric field vector. The local version of (11) and the associated boundary conditions can be written as

$$
\begin{aligned}
\boldsymbol{E}_{0} & =-\nabla_{0} \varphi & & \text { in } V \\
\varphi & =(\varphi)_{\partial_{\varphi}} V & & \text { on } \partial_{\varphi} V
\end{aligned}
$$

where $\varphi$ is the scalar electric potential. In (12), $\partial_{\varphi} V$ represents parts of the boundary $\partial V$ where essential electric potential boundary conditions are applied such that $\partial_{\omega} V \cup \partial_{\varphi} V=\partial V$ and $\partial_{\omega} V \cap \partial_{\varphi} V=\emptyset$. The spatial electric field vector $\boldsymbol{E}$ can be obtained by performing the push forward (standard fibre transformation) on material electric field i.e. $\boldsymbol{E}_{0}=\boldsymbol{F}^{T} \boldsymbol{E}$. 


\subsection{The internal energy density: Objective convex multi-variable electro-elasticity}

For the closure of the system of equations defined by (8), (10) and (12), two additional constitutive laws are needed relating deformation and electric displacements to stresses and electric fields in the continuum, satisfying appropriate constitutive inequalities, in particular ellipticity $[5,2]$. In the case of reversible electro-elasticity, where thermal effects and electric polarisation induced hysteresis are disregarded, the internal energy density $e$ per unit of undeformed volume can be solely defined in terms of the deformation and electric displacement, namely $e=e\left(\boldsymbol{\nabla}_{0} \boldsymbol{x}, \boldsymbol{D}_{0}\right)$. The requirement for objectivity (i.e. invariance with respect to rotations in the material configuration) implies that $e$ must be independent of the rotational components of deformation. This can be facilitated by re-expressing the internal energy density in terms of a symmetric kinematic measure (such as the symmetric right Cauchy-Green tensor $\boldsymbol{C}$ ) as follows

$$
e\left(\boldsymbol{\nabla}_{0} \boldsymbol{x}, \boldsymbol{D}_{0}\right)=e_{\mathrm{sym}}\left(\boldsymbol{C}, \boldsymbol{D}_{0}\right)
$$

Denoting the virtual and incremental variations of the electric displacements with $\delta \boldsymbol{D}_{0}$ and $\Delta \boldsymbol{D}_{0}$, respectively, that satisfy compatible homogeneous displacement, the first law of thermodynamics yields

$$
D e_{\mathrm{sym}}\left[\delta \boldsymbol{u} ; \delta \boldsymbol{D}_{0}\right]=\boldsymbol{S}: \frac{1}{2} D \boldsymbol{C}[\delta \boldsymbol{u}]+\boldsymbol{E}_{0} \cdot \delta \boldsymbol{D}_{0},
$$

where the second Piola-Kirchhoff stress tensor $\boldsymbol{S}$ and the Lagrangian electric field $\boldsymbol{E}_{0}$ are computed as

$$
\boldsymbol{S}=2 \frac{\partial e_{\mathrm{sym}}\left(\boldsymbol{C}, \boldsymbol{D}_{0}\right)}{\partial \boldsymbol{C}} ; \quad \quad \boldsymbol{E}_{0}=\frac{\partial e_{\mathrm{sym}}\left(\boldsymbol{C}, \boldsymbol{D}_{0}\right)}{\partial \boldsymbol{D}_{0}}
$$

wherein the first and second Piola-Kirchhoff tensors are related through $\boldsymbol{P}=\boldsymbol{F} \boldsymbol{S}$. Of particular importance to this development is multi-variable convexity in electromechanics, recently presented by Gil and Ortigosa [1, 2, 3], which automatically satisfies ellipticity. Using the convex multivariable definition, the internal energy density is postulated as

$$
e\left(\boldsymbol{\nabla}_{0} \boldsymbol{x}, \boldsymbol{D}_{0}\right)=W\left(\boldsymbol{F}, \boldsymbol{H}, J, \boldsymbol{D}_{0}, \boldsymbol{d}\right) ; \quad \boldsymbol{d}=\boldsymbol{F} \boldsymbol{D}_{0},
$$

where $W$ represents a convex multi-variable functional in terms of the extended set of arguments $\left\{\boldsymbol{F}, \boldsymbol{H}, J, \boldsymbol{D}_{0}, \boldsymbol{d}\right\}$. For the requirement of objectivity, the convex multi-variable energy $W$ can be re-expressed in terms of a set of symmetric kinematics defined in (3)

$$
e\left(\boldsymbol{\nabla}_{0} \boldsymbol{x}, \boldsymbol{D}_{0}\right)=e_{\mathrm{sym}}\left(\boldsymbol{C}, \boldsymbol{D}_{0}\right) \underset{\Leftarrow}{=} W\left(\boldsymbol{F}, \boldsymbol{H}, J, \boldsymbol{D}_{0}, \boldsymbol{d}\right) \underset{\Rightarrow}{\Rightarrow} W_{\mathrm{sym}}\left(\boldsymbol{C}, \boldsymbol{G}, C, \boldsymbol{D}_{0}\right),
$$

where $e_{\text {sym }}$ represents the internal energy in terms of the right Cauchy Green strain tensor $\boldsymbol{C}$ and Lagrangian electric displacement $\boldsymbol{D}_{0}$ and $W_{\text {sym }}$ represents an internal energy defined in terms of the extended symmetric mechanical kinematic set $\mathcal{V}_{\mathrm{sym}}^{m}=\{\boldsymbol{C}, \boldsymbol{G}, C\}$ and the Lagrangian electric displacement $\boldsymbol{D}_{0}$. It is worth noting that $W_{\text {sym }}$ is not strictly convex with respect to the individual components of the set $\left\{\mathcal{V}_{\text {sym }}^{m}, \boldsymbol{D}_{0}\right\}$, but rather an objective re-expression of the convex multi-variable functional $W$. Furthermore, the inclusion of the term $\boldsymbol{d}$ in $W$ is useful in the context of studying material stability [1] which can be re-expressed in terms of the combination of kinematics in $\left\{\mathcal{V}_{\text {sym }}^{m}, \boldsymbol{D}_{0}\right\}$. Notice that, constructing a convex multi-variable energy (i.e. $W$ ) 
is a necessary first step for a materially frame indifferent representation (i.e. $W_{\text {sym }}$ ), but the vice-versa is not necessarily true.

Definition of an objective internal energy density such as in (17) is an essential requirement for the particularisation of a multi-variable convex function to the case of small strains [38]. Furthermore, this definition of the internal energy $e_{\text {sym }}(17)$ facilitates the introduction of a new set of work-conjugates which can now be defined as

$$
\boldsymbol{\Sigma}_{\boldsymbol{C}}=2 \frac{\partial W_{\mathrm{sym}}}{\partial \boldsymbol{C}} ; \quad \boldsymbol{\Sigma}_{\boldsymbol{G}}=2 \frac{\partial W_{\mathrm{sym}}}{\partial \boldsymbol{G}} ; \quad \Sigma_{C}=2 \frac{\partial W_{\mathrm{sym}}}{\partial C} ; \quad \boldsymbol{\Sigma}_{\boldsymbol{D}_{0}}=\frac{\partial W_{\mathrm{sym}}}{\partial \boldsymbol{D}_{0}} .
$$

For notational convenience, the following sets, featuring in subsequent sections, are introduced

$$
\begin{aligned}
\mathcal{V}_{\text {sym }}^{m} & =\{\boldsymbol{C}, \boldsymbol{G}, C\} ; & \Sigma_{\mathcal{V}_{\text {sym }}^{m}}^{m} & =\left\{\boldsymbol{\Sigma}_{\boldsymbol{C}}, \boldsymbol{\Sigma}_{\boldsymbol{G}}, \Sigma_{C}\right\} ; \\
\mathcal{V}^{e} & =\left\{\boldsymbol{D}_{0}\right\} ; & \Sigma_{\mathcal{V}}^{e} & =\left\{\Sigma_{\boldsymbol{D}_{0}}\right\} ; \\
\mathcal{V} & =\left\{\mathcal{V}_{\text {sym }}^{m}, \mathcal{V}^{e}\right\} ; & \Sigma_{\mathcal{V}} & =\left\{\Sigma_{\mathcal{V}_{\text {sym }}}, \Sigma_{\mathcal{V}}^{e}\right\}
\end{aligned}
$$

Following [22, 1, 2], a physically more insightful representation for the second Piola-Kirchhoff stress tensor $\boldsymbol{S}$ and the Lagrangian electric field $\boldsymbol{E}_{0}$ can be obtained as

$$
\boldsymbol{S}=\Sigma_{C}+\Sigma_{G} \times C+\Sigma_{C} \boldsymbol{E} \quad \boldsymbol{E}_{0}=\Sigma_{D_{0}}
$$

Subsequently, the internal energy $e_{\text {sym }}=e_{\text {sym }}\left(\boldsymbol{C}, \boldsymbol{D}_{0}\right)$ can be further linearised leading to a tangent operator (facilitating a Newton-Raphson solution) which can be defined as follows

$$
D^{2} e_{\mathrm{sym}}\left[\delta \boldsymbol{u}, \delta \boldsymbol{D}_{0} ; \Delta \boldsymbol{u}, \Delta \boldsymbol{D}_{0}\right]=\left[\frac{1}{2} D \boldsymbol{C}[\delta \boldsymbol{u}]: \delta \boldsymbol{D}_{0}\right]\left[\begin{array}{cc}
\mathcal{C} & \mathcal{Q}^{T} \\
\mathcal{Q} & \boldsymbol{\theta}
\end{array}\right]\left[\begin{array}{c}
: \frac{1}{2} D \boldsymbol{C}[\Delta \boldsymbol{u}] \\
\Delta \boldsymbol{D}_{0}
\end{array}\right]+\boldsymbol{S}: \frac{1}{2} D^{2} \boldsymbol{C}[\delta \boldsymbol{u} ; \Delta \boldsymbol{u}]
$$

with the fourth order elasticity tensor $\mathcal{C}$, the third order coupling tensor $\mathcal{Q}$ and the second order dielectric tensor $\boldsymbol{\theta}$ defined as

$$
\mathcal{C}=4 \frac{\partial^{2} e_{\text {sym }}\left(\boldsymbol{C}, \boldsymbol{D}_{0}\right)}{\partial \boldsymbol{C} \partial \boldsymbol{C}} ; \quad \mathcal{Q}=2 \frac{\partial^{2} e_{\mathrm{sym}}\left(\boldsymbol{C}, \boldsymbol{D}_{0}\right)}{\partial \boldsymbol{D}_{0} \partial \boldsymbol{C}} ; \quad \boldsymbol{\theta}=\frac{\partial^{2} e_{\mathrm{sym}}\left(\boldsymbol{C}, \boldsymbol{D}_{0}\right)}{\partial \boldsymbol{D}_{0} \partial \boldsymbol{D}_{0}} .
$$

Analogous to (20), a more physically insightful representation of the tangent operator can be obtained as

$$
\begin{aligned}
D^{2} e_{\mathrm{sym}}[\delta \boldsymbol{u} ; \Delta \boldsymbol{u}] & =\left[\mathbb{M}_{\delta}\right]^{T}\left[\mathbb{H}_{W_{\mathrm{sym}}}\right]\left[\mathbb{M}_{\Delta}\right]+\left(\boldsymbol{\Sigma}_{\boldsymbol{G}}+\Sigma_{C} \boldsymbol{C}\right):\left(\frac{1}{2} D \boldsymbol{C}[\delta \boldsymbol{u}] \times \frac{1}{2} D \boldsymbol{C}[\Delta \boldsymbol{u}]\right) \\
& +\boldsymbol{S}:\left[\left(\boldsymbol{\nabla}_{0} \delta \boldsymbol{u}\right)^{T}\left(\boldsymbol{\nabla}_{0} \boldsymbol{\Delta} \boldsymbol{u}\right)\right]
\end{aligned}
$$

where

$$
\begin{aligned}
{\left[\mathbb{M}_{\delta}\right]^{T}=} & {\left[\frac{1}{2} D \boldsymbol{C}[\delta \boldsymbol{u}]: \frac{1}{2} D \boldsymbol{G}[\delta \boldsymbol{u}]: \frac{1}{2} D C[\delta \boldsymbol{u}] \quad \delta \boldsymbol{D}_{0} \cdot\right] } \\
{\left[\mathbb{M}_{\Delta}\right]=} & {\left[\begin{array}{c}
: \frac{1}{2} D \boldsymbol{C}[\Delta \boldsymbol{u}] \\
: \frac{1}{2} D \boldsymbol{G}[\Delta \boldsymbol{u}] \\
\frac{1}{2} D C[\Delta \boldsymbol{u}] \\
\boldsymbol{\Delta} \boldsymbol{D}_{0}
\end{array}\right] }
\end{aligned}
$$


with the Hessian operator $\left[\mathbb{H}_{W_{\text {sym }}}\right]$ defined as

$$
\left[\mathbb{H}_{W_{\mathrm{sym}}}\right]=\left[\begin{array}{cccc}
4 W_{\mathrm{sym}_{\boldsymbol{C}} \boldsymbol{C}} & 4 W_{\mathrm{sym}_{\boldsymbol{G}} \boldsymbol{G}} & 4 W_{\mathrm{sym}_{\boldsymbol{C}} C} & 2 W_{\mathrm{sym}_{\boldsymbol{C}} \boldsymbol{D}_{0}} \\
4 W_{\mathrm{sym}_{\boldsymbol{G}} \boldsymbol{C}} & 4 W_{\mathrm{sym}_{\boldsymbol{G}}} & 4 W_{\mathrm{sym}_{\boldsymbol{G} C}} & 2 W_{\mathrm{sym}_{\boldsymbol{G}} \boldsymbol{D}_{0}} \\
4 W_{\mathrm{sym}_{C} \boldsymbol{C}} & 4 W_{\mathrm{sym}_{C} \boldsymbol{G}} & 4 W_{\mathrm{sym}_{C C}} & 2 W_{\mathrm{sym}_{C} \boldsymbol{D}_{0}} \\
2 W_{\mathrm{sym}_{\boldsymbol{D}_{0} \boldsymbol{C}}} & 2 W_{\mathrm{sym}_{\boldsymbol{D}_{0} \boldsymbol{G}}} & 2 W_{\mathrm{sym}_{\boldsymbol{D}_{0} C}} & W_{\mathrm{sym}_{\boldsymbol{D}_{0} \boldsymbol{D}_{0}}}
\end{array}\right],
$$

where the components of $\mathcal{C}, \mathcal{Q}$ and $\boldsymbol{\theta}$ can now be defined in terms of the set of work-conjugates $\Sigma_{\mathcal{V}}$ defined in (19) using Table 1. It is important to note that, as opposed to the convex multi-

\begin{tabular}{|c|c|}
\hline $\mathcal{C}$ & $\begin{array}{l}W_{\mathrm{sym}_{C C}}+\boldsymbol{C} \times\left(W_{\mathrm{sym}_{G G}} \times \boldsymbol{C}\right)+\left(W_{\mathrm{sym}_{C C}} \boldsymbol{G} \otimes \boldsymbol{G}\right) \\
+W_{\mathrm{sym}_{C G}} \times \boldsymbol{C}+\boldsymbol{C} \times W_{\mathrm{sym}_{C G}} \\
+W_{\mathrm{sym}_{C C}} \otimes \boldsymbol{G}+\boldsymbol{G} \otimes W_{\mathrm{sym}_{C C}} \\
+\left(\boldsymbol{C} \times W_{\mathrm{sym}_{C C}}\right) \otimes \boldsymbol{G}+\boldsymbol{G} \otimes\left(W_{\mathrm{sym}_{C C}} \times \boldsymbol{C}\right)\end{array}$ \\
\hline $\mathcal{Q}^{T}$ & $W_{\mathrm{sym}_{C D_{0}}}+\boldsymbol{C} \times W_{\mathrm{sym}_{G D_{0}}}+\boldsymbol{G} \otimes W_{\mathrm{sym}_{C D_{0}}}$ \\
\hline$\theta$ & $W_{\boldsymbol{D}_{0} \boldsymbol{D}_{0}}$ \\
\hline
\end{tabular}

Table 1: Elasticity tensor $\mathcal{C}$, piezoelectric tensor $\mathcal{Q}$ and dielectric $\boldsymbol{\theta}$ tensor re-expressed in terms of the components of the Hessian operator.

variable Hessian operator expressed in terms of the fundamental kinematic set $\left\{\boldsymbol{F}, \boldsymbol{H}, J, \boldsymbol{D}_{0}, \boldsymbol{d}\right\}$ presented in [1], the constitutive term (first term) in (23) is not strictly positive definite as multivariable convexity is not defined with respect to the set $\mathcal{V}_{\mathrm{sym}}^{m}$ and, hence, ellipticity (i.e. rank-one convexity) of the internal energy based on this constitutive term alone cannot be established.

\subsection{The Helmholtz-like energy density and Legendre transformation}

In the case of pursuing a standard variational implementation via the finite element method, where the scalar electric potential is preferred as an unknown over the electric displacement field vector, it is typically preferred to work with the Helmholtz-like energy $\Phi=\Phi\left(\boldsymbol{C},-\nabla_{0} \varphi\right)$, defined as $[1,2]^{4}$

$$
\Phi\left(\boldsymbol{C},-\nabla_{0} \varphi\right)=-\sup _{\boldsymbol{D}_{0}}\left\{-\nabla_{0} \varphi \cdot \boldsymbol{D}_{0}-e_{\mathrm{sym}}\left(\boldsymbol{C}, \boldsymbol{D}_{0}\right)\right\}
$$

\footnotetext{
${ }^{4}$ The usage of Helmholtz-like energy is due to the fact that the Helmholtz energy is predominantly used in the context of thermal problems for the free energy expressed in terms temperature and the Legendre transformation therein to obtain the internal energy expressed in terms of entropy.
} 
wherein the second Piola-Kirchhoff stress tensor $\boldsymbol{S}$ and the Lagrangian electric displacement $\boldsymbol{D}_{0}$ are computed as

$$
\boldsymbol{S}=\left.2 \frac{\partial \Phi\left(\boldsymbol{C}, \boldsymbol{E}_{0}\right)}{\partial \boldsymbol{C}}\right|_{\boldsymbol{E}_{0}=-\nabla_{0} \varphi} ; \quad \boldsymbol{D}_{0}=-\left.\frac{\partial \Phi\left(\boldsymbol{C}, \boldsymbol{E}_{0}\right)}{\partial \boldsymbol{E}_{0}}\right|_{\boldsymbol{E}_{0}=-\nabla_{0} \varphi} .
$$

Further linearisation of the Helmholtz-like energy leads to the three constitutive tensors analogous to $(22)$

$$
\mathcal{C}_{\Phi}=\left.\frac{\partial^{2} \Phi\left(\boldsymbol{C}, \boldsymbol{E}_{0}\right)}{\partial \boldsymbol{C} \partial \boldsymbol{C}}\right|_{\boldsymbol{E}_{0}=-\nabla_{0} \varphi} ; \quad \boldsymbol{\mathcal { Q }}_{\Phi}=\left.\frac{\partial^{2} \Phi\left(\boldsymbol{C}, \boldsymbol{E}_{0}\right)}{\partial \boldsymbol{C} \partial \boldsymbol{E}_{0}}\right|_{\boldsymbol{E}_{0}=-\nabla_{0} \varphi} ; \quad \boldsymbol{\theta}_{\Phi}=\left.\frac{\partial^{2} \Phi\left(\boldsymbol{C}, \boldsymbol{E}_{0}\right)}{\partial \boldsymbol{E}_{0} \partial \boldsymbol{E}_{0}}\right|_{\boldsymbol{E}_{0}=-\nabla_{0} \varphi},
$$

where the subscript $\boldsymbol{E}_{0}=-\nabla_{0} \varphi$ implies that energy is evaluated for the value of electric field obtained using (12). When the internal energy is a complex multi-variable function of the set $\mathcal{V}$, the nonlinearity of the convex multi-variable function $e_{\text {sym }}$ can make it impossible to obtain an explicit representation of the constitutive tensors in (29). Whence, it is typically more suitable to perform this step numerically, by utilising a nonlinear iterative scheme. In the context of finite element analysis, by exploiting the relationship between the internal energy and the Helmholtz-like energy through the Legendre transform in (27), it is possible to compute, via a Newton-Raphson algorithm per quadrature point, these tensors as

$$
\left[\boldsymbol{\theta}_{\Phi}\right]_{i j}=-[\boldsymbol{\theta}]_{i j}^{-1} ; \quad\left[\mathcal{Q}_{\Phi}\right]_{i j k}=-\left[\boldsymbol{\theta}_{\Phi}\right]_{m i}[\mathcal{Q}]_{j k m} ; \quad\left[\mathcal{C}_{\Phi}\right]_{i j k l}=[\mathcal{C}]_{i j k l}-[\mathcal{Q}]_{i j m}\left[\mathcal{Q}_{\Phi}\right]_{m k l} .
$$

For a detailed representation of different electromechanical energies, the reader can refer to Gil and Ortigosa [1].

\section{Variational formulation}

\subsection{Displacement-electric potential based variational formulation}

A variational principle can be established by the total energy minimisation defined in terms of the internal energy of the system $e_{\mathrm{sym}}=e_{\mathrm{sym}}\left(\boldsymbol{C}, \boldsymbol{D}_{0}\right)$. In this case, the total potential energy $\Pi_{e}\left(\boldsymbol{x}^{*}, \varphi^{*}, \boldsymbol{D}_{0}^{*}\right)$ can be written as

$$
\Pi_{e}\left(\boldsymbol{x}^{*}, \varphi^{*}, \boldsymbol{D}_{0}^{*}\right)=\inf _{\boldsymbol{x}, \boldsymbol{D}_{0}} \sup _{\varphi}\left\{\int_{V} e_{\mathrm{sym}}\left(\boldsymbol{C}, \boldsymbol{D}_{0}\right) d V+\int_{V} \boldsymbol{D}_{0} \cdot \nabla_{0} \varphi d V-\Pi_{\mathrm{ext}}(\boldsymbol{x}, \varphi)\right\},
$$

where $\left(\boldsymbol{x}^{*}, \varphi^{*}, \boldsymbol{D}_{0}^{*}\right)$ denotes the exact solution and $\Pi_{\text {ext }}(\boldsymbol{x}, \varphi)$ represents the external coupled electromechanical work additively decomposed into the purely mechanical $\Pi_{\text {ext }}^{m}(\boldsymbol{x})$ and electrical $\Pi_{\text {ext }}^{e}(\varphi)$ components

$$
\begin{aligned}
& \Pi_{\mathrm{ext}}^{m}(\boldsymbol{x})=\int_{V} \boldsymbol{f} \cdot \boldsymbol{x} d V+\int_{\partial_{t} V} \boldsymbol{t}_{0} \cdot \boldsymbol{x} d A \\
& \Pi_{\mathrm{ext}}^{e}(\varphi)=-\int_{V} \rho_{0}^{e} \varphi d V-\int_{\partial_{\omega} V} \omega_{0}^{e} \varphi d A
\end{aligned}
$$

where

$$
\Pi_{\mathrm{ext}}(\boldsymbol{x}, \varphi)=\Pi_{\mathrm{ext}}^{m}(\boldsymbol{x})+\Pi_{\mathrm{ext}}^{e}(\varphi)
$$


Application of the Legendre transform enables [1] above variational principle (31) to be reformulated as

$$
\Pi_{\Phi}\left(\boldsymbol{x}^{*}, \varphi^{*}\right)=\inf _{\boldsymbol{x}} \sup _{\varphi}\left\{\int_{V} \Phi\left(\boldsymbol{C},-\nabla_{0} \varphi\right) d V-\Pi_{\mathrm{ext}}(\boldsymbol{x}, \varphi)\right\} .
$$

It is now straightforward to carry out finite element discretisation of (32) in terms of $\{\boldsymbol{x}, \varphi\}$, for which we refer to [2]. In this context, (32) can be solved for in a standard monolithic way as described in Algorithm 1. In the current setting, equal order high order isoparametric finite elements are utilised for the interpolation of the primary variables i.e. $\{\boldsymbol{x}, \varphi\}$.

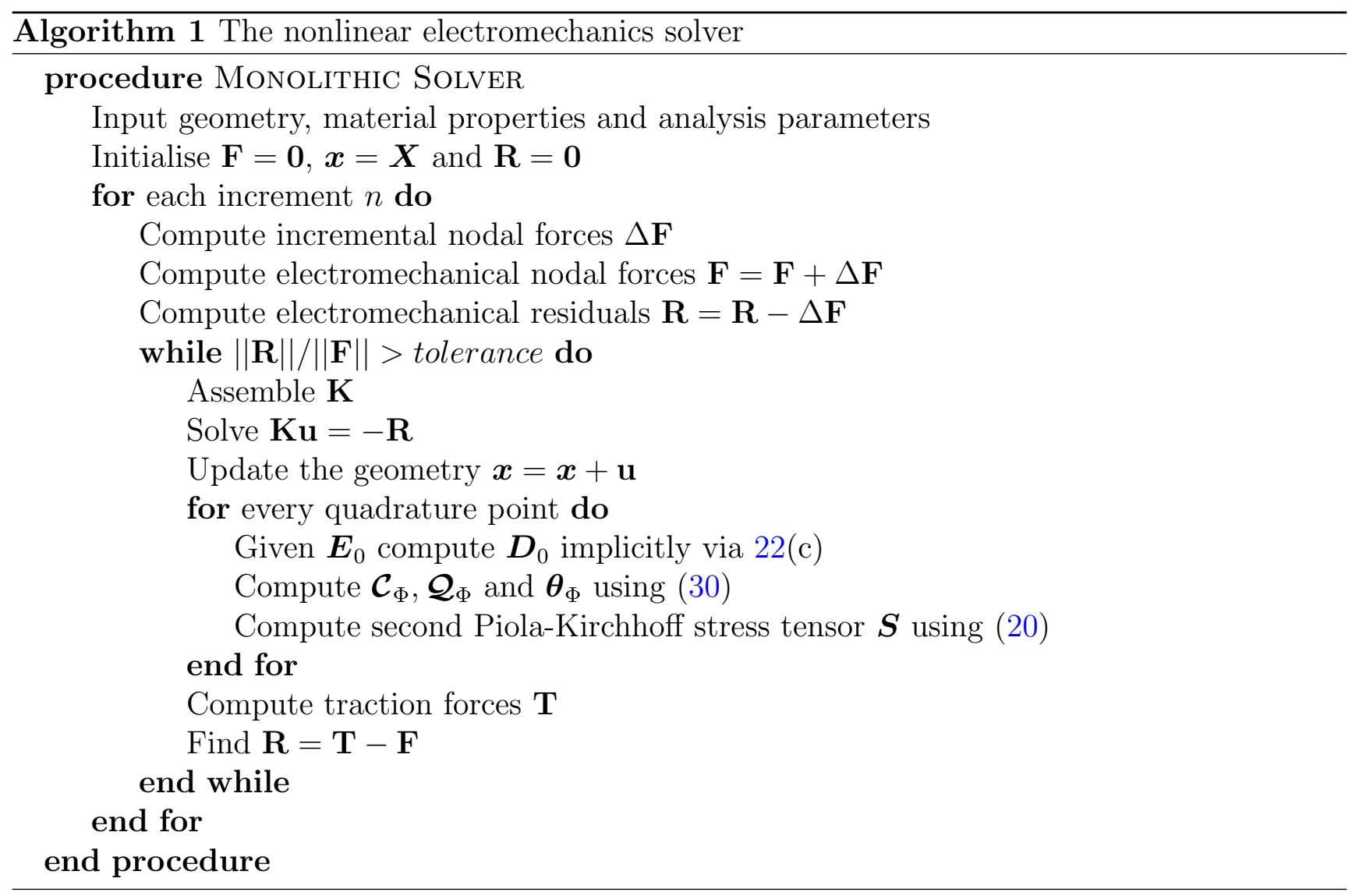

\subsection{Incrementally linearised electromechanics}

As presented in section 1, a particular class of coupling in electromechanics deals with small strains coupled with nonlinear electrostatics. To this end, the variational principle described in (32) can be particularised for the case of small strains. Extending, the strategy presented in $[38,42]$, let us consider the total potential energy (32) cast in an iterative (Newton-Raphson) form

$$
\Pi_{\Phi_{\mathrm{int}}}\left(\boldsymbol{x}_{k+1}^{*^{n+1}}, \varphi_{k+1}^{*^{n+1}}\right)=\int_{V} \Phi\left(\boldsymbol{C}_{k+1}^{n+1},-\left(\nabla_{0} \varphi\right)_{k+1}^{n+1}\right) d V
$$

where superscripts denote increments and subscripts denote iterations. A geometrically linearised formulation for electro-elasticity can be obtained by perturbing the potential energy (33). Ho- 
wever note that in contrast to the approach presented in [38], particularisation of (33) for small strains through consistent linearisation with respect to the variations in electrostatic fields and mechanical fields cannot be performed simultaneously, as it leads to high order tensorial quantities without clear physical interpretations. However, as shown in Figure 2, for small deformations, the geometry could be solved for incrementally with only the electrostatic equations requiring a full Newton-Raphson procedure. This gives rise to the staggered scheme presented in the following subsection.

\subsection{The staggered approach to incrementally linearised electromechanics}

To elaborate the staggered approach emanating from the variational principle (31), let us reconsider (33) which in fully discretised form (using the finite element method) can be written as

$$
\left[\begin{array}{rr}
\mathbf{K}_{\mathbf{u u}_{k+1}}^{n+1} & \mathbf{K}_{\mathbf{u} \phi_{k+1}^{n+1}}^{n+1} \\
\mathbf{K}_{\phi \mathbf{u}_{k+1}}^{n+1} & \mathbf{K}_{\phi \phi_{k+1}^{n+1}}^{n+1}
\end{array}\right]\left[\begin{array}{r}
\mathbf{U}_{\mathbf{u}_{k+1}^{n+1}} \\
\mathbf{U}_{\phi_{k+1}^{n+1}}^{n+1}
\end{array}\right]=-\left[\begin{array}{l}
\mathbf{R}_{\mathbf{u}_{k+1}^{n+1}} \\
\mathbf{R}_{\phi_{k+1}^{n+1}}^{n+1}
\end{array}\right],
$$

where $\mathbf{K}_{\mathbf{u u}}$ represents the fully discrete purely mechanical stiffness matrix, $\mathbf{K}_{\phi \phi}$ the fully discrete matrix associated with electrostatic variable(s) and $\mathbf{K}_{\mathbf{u} \phi}$ and $\mathbf{K}_{\boldsymbol{\phi} \mathbf{u}}$ the fully discrete electromechanical coupled matrices. The above discrete form particularised to the staggered case of (31) can be established as shown in Algorithm 2. Algorithm 2 in particular implies a staggered system in which the geometry is updated incrementally, but the associated electrostatic variables are solved for iteratively. More specifically, as shown in Figure 2 the geometry, the mechanical and the coupling matrices are frozen during the iterative (implicit) solution of the electrostatic variables. An algorithmic representation of this staggered scheme is presented in Algorithm 2 where $\mathbf{N}$ represents the external electrical nodal force vector. Note that an initial step for solving the mechanical variables is necessary in this case i.e. $\mathbf{K}_{\mathbf{u u} 00}^{0} \mathbf{U}_{\mathbf{u} 0}^{0}=-\mathbf{R}_{\mathbf{u} 0}^{0}$ to account for Dirichlet driven problems. Since the above staggered approach requires the solution of a scalar field electric potential through solving the discretised Gauss's law, the saving in computational cost can be tremendous within its range of applicability (small strains). However as the voltage induced deformation increases a high number of increments might be needed to obtain the results of fully nonlinear monolithic approach.

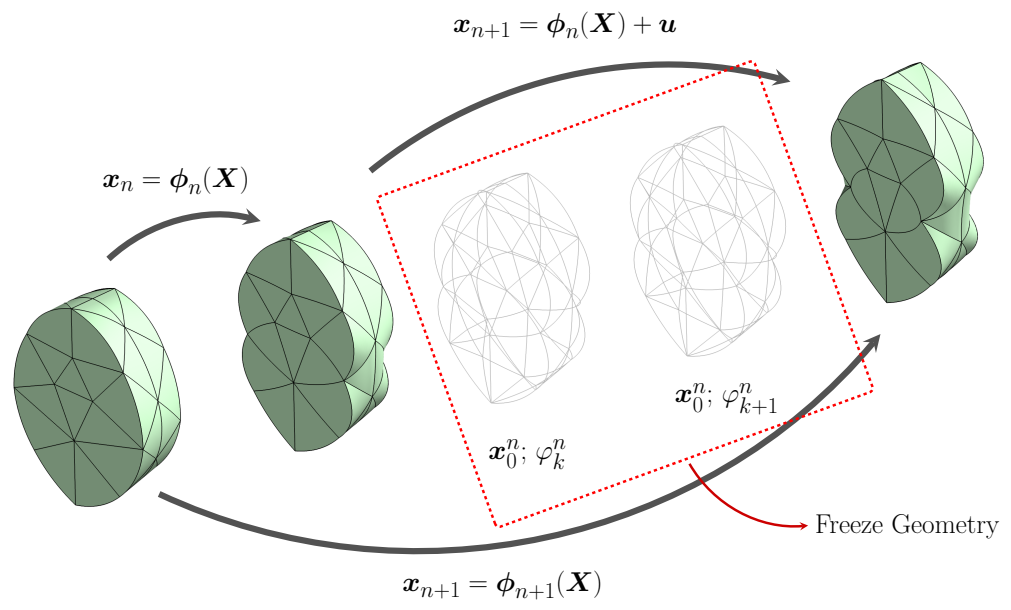

Figure 2: Schematic representation of the staggered incrementally linearised scheme. 


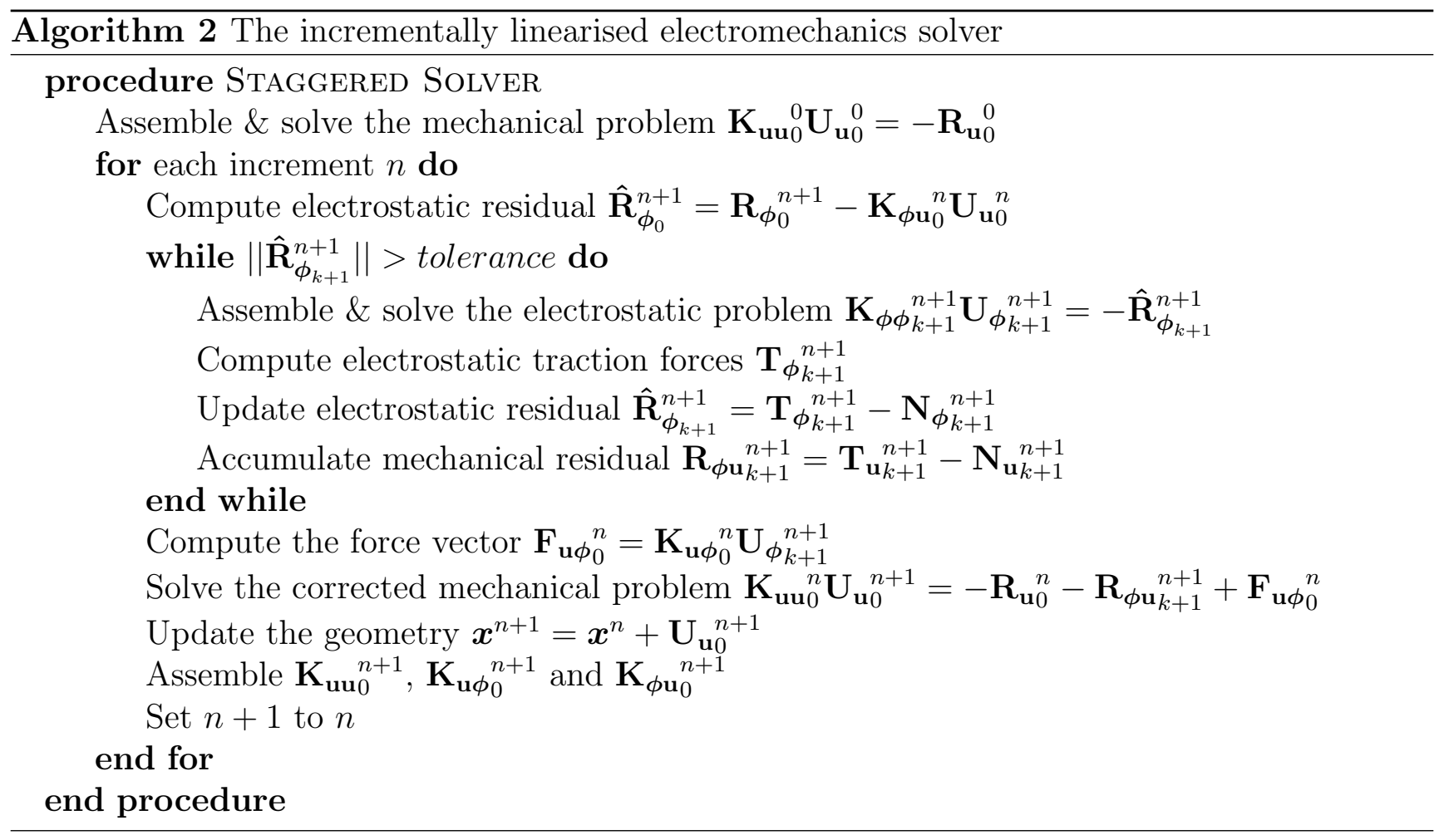

\section{Numerical examples}

In this section a series of numerical examples for electromechanics are presented. These include a) (mesh refinement) $h$ and (polynomial enrichment) $p$ convergence studies for high order displacement-potential formulation for convex multi-variable internal energies presented in section $3, \mathrm{~b}$ ) the impact of accurate geometrical representations on the solution of large deformation electromechanical problems, c) comparison of monolithic (nonlinear) approach with the incrementally linearised staggered approach and the range of applicability of the latter approach in actuation, d) comparison of the current framework with the mixed Hu-Washizu variational principles presented in $[2,16]$ and, e) Impact of code transformation and data parallelism for an efficient implementation of convex multi-variable electro-elasticity in the context of high order finite elements. Apart from these benchmark studies, a series of examples pertaining to the massive deformation and the instabilities in DEs such as the formation of folds and wrinkles is studied in detail using the current framework. The finite element implementation involves equal order standard isoparametric discretisation of the electromechanical variables $\{\boldsymbol{x}, \varphi\}$, starting with at least quadratic basis functions. As a standard nomenclature in high order finite element analysis, polynomial interpolation over tetrahedra are denoted by $p$ and polynomial enrichment over hexahedra are denoted by $q$. Unless otherwise specified, for all the examples, the curvilinear meshes are generated using the consistently linearised solid mechanics analogy presented in $[38,43]$, using a Mooney-Rivlin model with a Poisson's ratio of 0.45 and 10 load increments. In addition, all the analyses are carried out with the high performance domain-specific data parallel tensor contraction finite element framework Fastor based on the authors' previous work [44] and the massively parallel multi-frontal direct sparse solver MUMPS is used for the solution of system of linear equations. For the purpose of assessing the performance of the proposed monolithic approach the final example is solved using the algebraic multigrid (AMG) solver with the standard 
Ruge-Stuben aggregations, c.f. [45, 46, 47, 48].

\section{1. $h$ \& $p$ convergence of the proposed high order framework}

As a starting point, it is essential to examine the $h$ and $p$ convergence properties of the proposed high order displacement-potential formulation for large deformations large electric fields (fully coupled monolithic approach) described in section 2 and subsection 3.1. Hence, the objective of this example is to a) assess the convergence of different variables in $\left\{\boldsymbol{x}, \boldsymbol{\varphi}, \mathcal{V}, \Sigma_{\mathcal{V}}\right\}$ using $h$ and $p$ refinements, b) examine optimality of the algorithm in terms of convergence properties on high order curved tetrahedral and hexahedral meshes around a dielectric elastomeric patch, obtained using the mesh deformation technique described in [38] and, c) showcase the scalability of the framework with high polynomial enrichment. The geometry of the dielectric patch is shown in Figure 3. A similar convergence study for an eleven field $\mathrm{Hu}$-Washizu type mixed variational formulation in terms of the set of unknowns $\left\{\boldsymbol{x}, \boldsymbol{F}, \boldsymbol{H}, J, \varphi, \boldsymbol{d}, \boldsymbol{\Sigma}_{\boldsymbol{F}}, \boldsymbol{\Sigma}_{\boldsymbol{H}}, \Sigma_{J}, \boldsymbol{\Sigma}_{\boldsymbol{D}_{0}}, \boldsymbol{\Sigma}_{\boldsymbol{d}}\right\}$ is presented in [2]. The constitutive model considered is based on a convex multi-variable energy functional expressed in terms of the invariants of the set $\mathcal{V}$ as follows

$$
\begin{aligned}
W_{e l, 1}\left(\boldsymbol{C}, \boldsymbol{G}, C, \boldsymbol{D}_{0}\right)=\mu_{1} \sum_{i=1}^{n} \alpha_{i} I_{\boldsymbol{C}}^{i}+\mu_{2} \sum_{i=1}^{n} \beta_{i} I_{\boldsymbol{G}}^{i}-2 & \left(\mu_{1} \sum_{i=1}^{n} i \alpha_{i} 3^{i-1}+2 \mu_{2} \sum_{i=1}^{n} i \beta_{i} 3^{i-1}\right) \ln \sqrt{C} \\
& +\frac{\lambda}{2}(\sqrt{C}-1)^{2}+\frac{1}{2 \varepsilon_{1}} I I_{\boldsymbol{D}_{0}}+\frac{1}{2 \varepsilon_{2} \sqrt{C}} I I_{\boldsymbol{d}}
\end{aligned}
$$

where $I_{(\bullet)}$ denotes the trace of the entity $(\bullet)$ and $I I_{(\bullet)}$ the squared of the $\mathcal{L}^{2}$ norm of the entity $(\bullet)$ with $I I_{\boldsymbol{d}}=\boldsymbol{D}_{0} \cdot \boldsymbol{C} \boldsymbol{D}_{0}$. Furthermore, $\left\{\mu_{1}, \mu_{2}, \lambda, \varepsilon_{1}, \varepsilon_{2}\right\}$ represent positive material constants with $\alpha_{i}$ 's and $\beta_{i}$ 's denoting scaling coefficients. The material parameters in (35) used for this example are presented in Table 2, where $n=2, \alpha_{1}=\beta_{1}=1$ and $\alpha_{2}=\beta_{2}=0.2$ are chosen. The

\begin{tabular}{|l|l|l|l|l|}
\hline$\mu_{1}(\mathrm{~Pa})$ & $\mu_{2}(\mathrm{~Pa})$ & $\lambda(\mathrm{Pa})$ & $\varepsilon_{1}\left(\mathrm{~N} / \mathrm{V}^{2}\right)$ & $\varepsilon_{2}\left(\mathrm{~N} / \mathrm{V}^{2}\right)$ \\
\hline 1 & $1 / 2$ & 1 & 4 & 4 \\
\hline
\end{tabular}

Table 2: Material properties for example 4.1

problem is constructed so that smoothness of the solution is guaranteed. For that purpose, the following simple exact fields associated with the fields $\boldsymbol{x}$ and $\varphi$ are considered

$$
\boldsymbol{x}^{\text {exact }}=\boldsymbol{X}+\left\{\begin{array}{c}
\gamma_{1} \sin X_{1} \\
\gamma_{2} \cos X_{2} \\
\gamma_{3}\left(\sin X_{3}+\cos X_{3}\right)
\end{array}\right\} ; \quad \varphi^{\text {exact }}=\varphi_{0} \sin X_{1},
$$

where for this benchmark example, $\gamma_{1}=0.1, \gamma_{2}=0.2, \gamma_{3}=0.3$ and $\varphi_{0}=10^{4} V$ are chosen. The deformation gradient tensor and the Lagrangian electric field can now be computed as

$$
\boldsymbol{F}^{\text {exact }}=\left[\begin{array}{ccc}
1+\gamma_{1} \cos X_{1} & 0 & 0 \\
0 & 1-\gamma_{2} \sin X_{2} & 0 \\
0 & 0 & 1+\gamma_{3}\left(\cos X_{3}-\sin X_{3}\right)
\end{array}\right] ; \quad \boldsymbol{E}_{0}^{\text {exact }}=-\left\{\begin{array}{c}
\varphi \cos X_{1} \\
0 \\
0
\end{array}\right\} \text {. }
$$


The remaining exact fields in the set $\mathcal{V}^{\text {exact }}=\left\{\boldsymbol{C}^{\text {exact }}, \boldsymbol{G}^{\text {exact }}, C^{\text {exact }}, \boldsymbol{D}_{0}^{\text {exact }}\right\}$ can now be obtained for the smooth displacement and electric potential fields, from (37). Application of (3) on (37) yields the exact right Cauchy-Green tensor, its cofactor and determinant for the smooth fields (36) as

$$
\boldsymbol{C}^{\text {exact }}=\boldsymbol{F}^{\text {exact } T} \boldsymbol{F}^{\text {exact }} ; \quad \boldsymbol{G}^{\text {exact }}=\frac{1}{2} \boldsymbol{C}^{\text {exact }} \times \boldsymbol{C}^{\text {exact }} ; \quad C^{\text {exact }}=\frac{1}{3} \boldsymbol{G}^{\text {exact }}: \boldsymbol{C}^{\text {exact }} .
$$

Similarly, the Lagrangian electric displacement vector can be computed by applying (15) on (35) as

$$
\boldsymbol{D}_{0}^{\text {exact }}=\left(\frac{1}{\varepsilon_{1}} \boldsymbol{I}+\frac{1}{\varepsilon_{2} \sqrt{C_{\text {exact }}}} \boldsymbol{C}^{\text {exact }}\right)^{-1} \boldsymbol{E}_{0}^{\text {exact }} .
$$

Once all the elements of the set $\mathcal{V}^{\text {exact }}$ have been determined, it is possible to obtain the set of exact work conjugates $\Sigma_{\mathcal{V}}^{\text {exact }}=\left\{\boldsymbol{\Sigma}_{\boldsymbol{C}}{ }^{\text {exact }}, \boldsymbol{\Sigma}_{\boldsymbol{G}}{ }^{\text {exact }}, \Sigma_{C}^{\text {exact }}, \boldsymbol{\Sigma}_{\boldsymbol{D}_{0}}^{\text {exact }}\right\}$ via (18). These enable to compute the second Piola-Kirchhoff stress tensor $\boldsymbol{S}^{\text {exact }}$ from (20). Finally, the associated volumetric force and electric charge in mechanical and electrical equilibrium with the exact first Piola-Kirchhoff stress tensor $\boldsymbol{P}^{\text {exact }}=\boldsymbol{F}^{\text {exact }} \boldsymbol{S}^{\text {exact }}$ and exact Lagrangian electric displacement field $\boldsymbol{D}_{0}^{\text {exact }}$ are determined from (8) and (10), respectively as

$$
\boldsymbol{f}_{0}\left(\boldsymbol{x}^{\text {exact }}, \varphi^{\text {exact }}\right)=-\mathrm{DIV} \boldsymbol{P}^{\text {exact }} ; \quad \rho_{0}\left(\boldsymbol{x}^{\text {exact }}, \varphi^{\text {exact }}\right)=\operatorname{DIV} \boldsymbol{D}_{0}^{\text {exact }}
$$

For the convergence studies, three different high order curvilinear unstructured tetrahedral meshes and one high order curvilinear structured hexahedral mesh for the dielectric patch in Figure 3 are considered with 532 elements, 9220 elements, 26807 elements for the first three and 5000 elements for the last one, respectively, as shown in Figure 5. As will be described shortly, these discretisations are chosen such that the maximum segment fitting in a curved element (denoted by $h$ ) is successively refined for $h$-convergence. The placements of high order nodes on curved boundaries of the meshes are given particular importance, in that they have been computed through an arc-length based projection from high order planar meshes with Warburton nodal distribution [38]. The curved volume mesh is then obtained by applying the consistently linearised elastic analogy $[38,43,49,50]$. It is worth mentioning that the quality of the curvilinear meshes directly impact the $h$ and $p$ convergence property (optimality) of the finite element interpolation scheme. To this end, Table 3 and Table 4 report the three fundamental quality measures (where $Q_{1}$ quantifies distortion of edges, $Q_{2}$ quantifies distortion of faces and $Q_{3}$, also known as scaled Jacobian, quantifies volumetric distortion of the element itself) of the curved tetrahedral and hexahedral meshes, respectively; c.f. [38]. Optimal symmetric quadrature rules for tetrahedra

\begin{tabular}{|c|c|c|c|c|c|c|c|c|c|c|c|c|}
\hline \multirow[b]{2}{*}{$p$} & \multicolumn{4}{|c|}{ Coarse (532 elements) } & \multicolumn{4}{|c|}{ Medium (9220 elements) } & \multicolumn{4}{|c|}{ Fine (26807 elements) } \\
\hline & DoFs & $Q_{1}$ & $Q_{2}$ & $Q_{3}$ & DoFs & $Q_{1}$ & $Q_{2}$ & $Q_{3}$ & DoFs & $Q Q_{1}$ & $Q_{2}$ & $Q_{3}$ \\
\hline$p=2$ & $1027 \times 4$ & 0.888 & 0.875 & 0.790 & $17934 \times 4$ & 0.962 & 0.924 & 0.885 & $52590 \times 4$ & 0.953 & 0.909 & 0.866 \\
\hline$p=3$ & $3077 \times 4$ & 0.881 & 0.843 & 0.746 & $54018 \times 4$ & 0.936 & 0.869 & 0.799 & $158352 \times 4$ & 0.947 & 0.893 & 0.838 \\
\hline$p=4$ & $6861 \times 4$ & 0.874 & 0.813 & 0.738 & $120473 \times 4$ & 0.926 & 0.850 & 0.771 & $352838 \times 4$ & 0.935 & 0.871 & 0.800 \\
\hline$p=5$ & $12911 \times 4$ & 0.851 & 0.807 & 0.711 & $226516 \times 4$ & 0.904 & 0.831 & 0.721 & $662848 \times 4$ & 0.924 & 0.843 & 0.765 \\
\hline$p=6$ & $21759 \times 4$ & 0.846 & 0.771 & 0.652 & $381373 \times 4$ & 0.891 & 0.828 & 0.713 & $1115196 \times 4$ & 0.901 & 0.837 & 0.757 \\
\hline
\end{tabular}
reported in [51] are utilised for numerical integration of high order tetrahedral elements. For hexahedral elements, a simple tensor product based quadrature rule is utilised.

Table 3: Distortion quality of high order curvilinear tetrahedral meshes 


\begin{tabular}{|l|l|l|l|l|}
\hline$p$ & DoFs & $Q_{1}$ & $Q_{2}$ & $Q_{3}$ \\
\hline$p=2$ & $4943 \times 4$ & 0.999 & 0.984 & 0.914 \\
\hline$p=3$ & $147364 \times 4$ & 0.993 & 0.972 & 0.889 \\
\hline$p=4$ & $341885 \times 4$ & 0.987 & 0.961 & 0.853 \\
\hline$p=5$ & $659106 \times 4$ & 0.981 & 0.934 & 0.820 \\
\hline$p=6$ & $1129027 \times 4$ & 0.971 & 0.900 & 0.797 \\
\hline
\end{tabular}

Table 4: Distortion quality of high curvilinear hexahedral mesh

For studying $h$-convergence properties, only tetrahedral meshes are considered. The convergence rate of different primary and derived variables $\left\{\boldsymbol{x}, \varphi, \mathcal{V}, \Sigma_{\mathcal{V}}\right\}$ are then studied by comparing the interpolated solution and analytical solution for a fixed $p$ and successive $h$-refinement, similar to the strategy followed in $[38,2]$. To monitor the convergence rate, the $\mathcal{L}^{2}(V)$ norm of the error is computed for all quantities of interest. For curvilinear meshes the $\mathcal{L}^{2}(V)$ norms of the variables

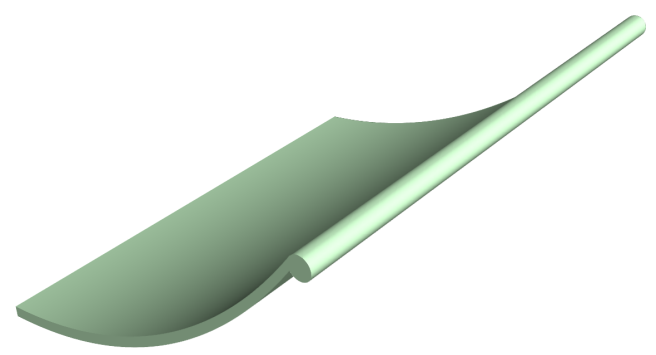

Figure 3: CAD geometry of the dielectric patch

are reported as a function of the diameter (the largest segment that can fit within a curved region) of the element computed through a straightforward sampling strategy illustrated in Figure 4 for curved tetrahedral and hexahedral elements. After traversing the whole computational mesh, the minimum value of all the diameters is chosen as $h[52,53]$. The tetrahedral meshes reported in Table 3 are chosen such that they correspond to successive refinement in this quantity.

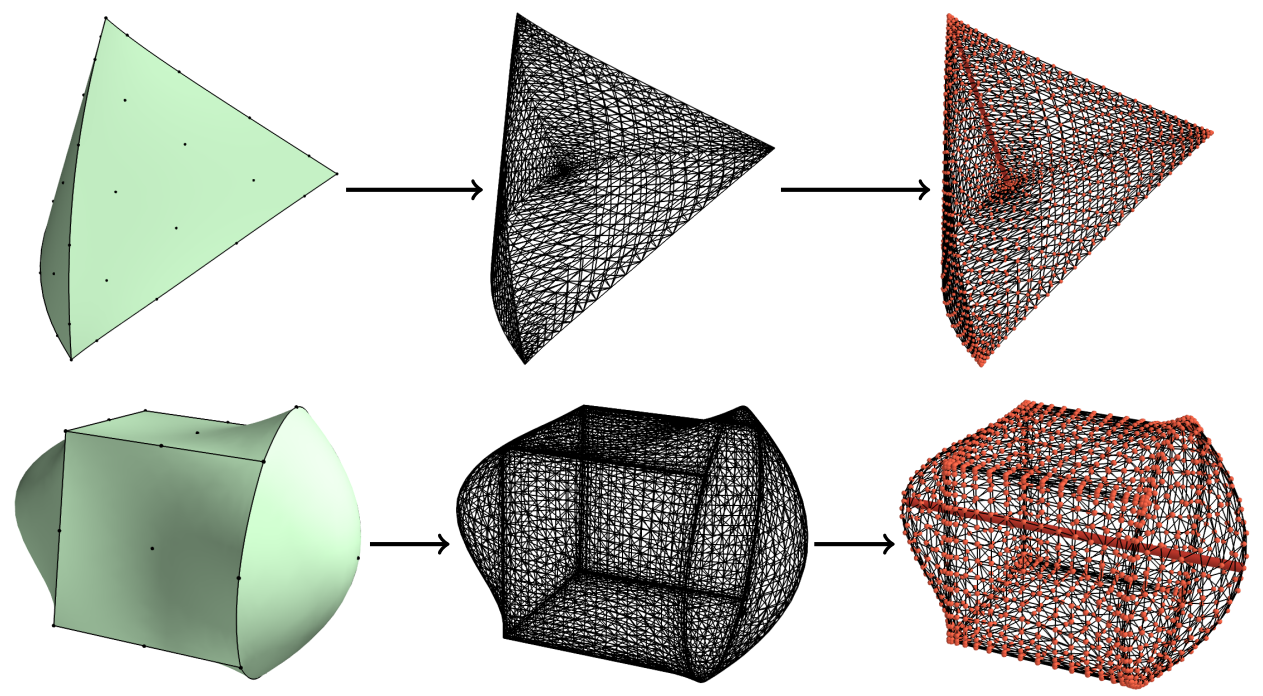

Figure 4: Illustration of computing the diameter (largest segment) in curvilinear meshes using sampling and tessellation strategy, for $p=5$ tetrahedra and $q=2$ hexahedra

Figure 6 shows $h$-convergence of the variables $\left\{\boldsymbol{x}, \varphi, \mathcal{V}, \Sigma_{\mathcal{V}}\right\}$ for four levels of $p$-refinement (i.e. $p=2,3,4,5)$. Note that unlike in [2], while the displacements and electric potential are primary 
(solved) variables, the rest of variables are secondary (derived). As can be observed in Figure 6 for

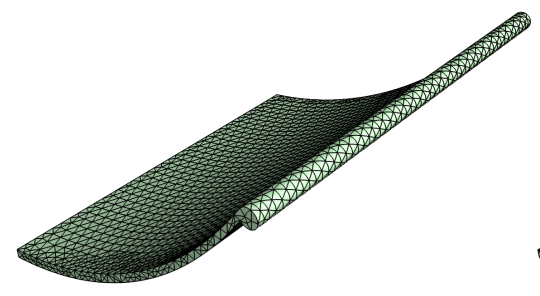

(a)

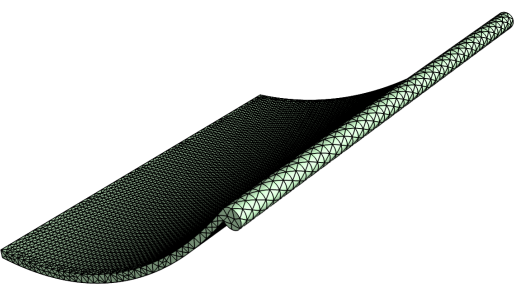

(b)

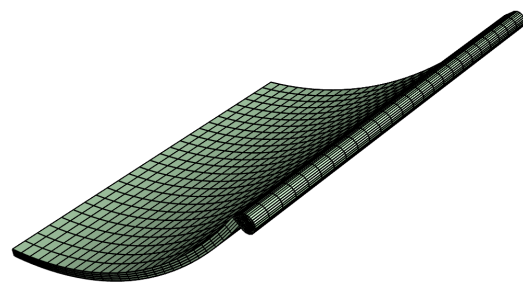

(c)

Figure 5: Three representative curvilinear meshes used for convergence studies; a) medium tetrahedral mesh (9220 elements) b) fine tetrahedral mesh (26807 elements), and c) fine hexahedral mesh (5000 elements)

both low and high order polynomial interpolations, the expected rate of convergence is achieved for both primary ( $p+1$ convergence rate) and derived ( $p$ convergence rate) variables. Notice that for this study, the multi-precision floating point library (MPFR) is utilised for arbitrary floating point accuracy. Apart from pinpointing the optimality and accuracy of the high order electromechanical solver on complex curved meshes, the $h$-convergence plots also prove that the framework scales reliably with high $p$ and millions of degrees of freedom. Next, the $p$-convergence of tetrahedral elements for a fixed mesh (the finest mesh) is considered and compared to the $p$-convergence properties of hexahedral elements. Notice from Table 3 and Table 4 that the hexahedral mesh is chosen such that for every $p$, the number of nodes in the tetrahedral and hexahedral meshes are within a $5 \%$ difference. Once again, the degree of interpolation is successively increased leading to $p$ convergence of the $\mathcal{L}^{2}(V)$ norm of the same afore-mentioned quantities. Figure 7 shows $p$ convergence of the $\mathcal{L}^{2}(V)$ for arguments of the set $\left\{\boldsymbol{x}, \varphi, \mathcal{V}, \Sigma_{\mathcal{V}}\right\}$. Once again, optimal rates of convergence for both curvilinear tetrahedral and hexahedral elements are obtained.

\subsection{Effect of accurate boundary representation in nonlinear electro-elasticity}

Having studied the convergence properties of high order curvilinear finite elements for modelling convex multi-variable electromechanics in the previous section, the objective of this section is to examine if accurate geometrical representation through high order curvilinear finite elements, does play a role in capturing the physics of soft elastomeric materials better compared to the standard high order finite elements with planer faces/edges. The examples in this section are also motivated by the fact that, the vast majority of finite element simulations of electromechanical devices are carried out using either low order or high order finite elements with planer faces $[54,55,56,2,16,23,34]$, with possible geometrical simplifications and at times even de-featuring [57].

To this end, the notable example of electromechanical plate with a hole is chosen for examination [54]. For this example, once again, one tetrahedral mesh and one hexahedral mesh is chosen and the polynomial degree is successively enriched, while keeping the mesh size $h$ fixed. Additionally, for both examples, the material model chosen is based on the following convex multi-variable strain energy representation

$$
W_{e l, 2}=\mu_{1} I_{\boldsymbol{C}}+\mu_{2} I_{\boldsymbol{G}}-2\left(\mu_{1}+\mu_{2}\right) \ln \sqrt{C}+\frac{\lambda}{2}(\sqrt{C}-1)^{2}+\frac{1}{2 \varepsilon_{1}} I I_{\boldsymbol{D}_{0}}+\frac{1}{2 \sqrt{C} \varepsilon_{2}} I I_{\boldsymbol{d}},
$$

with material constants as given in Table 5. The Poisson's ratio corresponding to parameters $\mu_{1}$, $\mu_{2}$ and $\lambda$, is $\nu=0.357$. The mesh quality information for the plate with the hole is listed in Table 6 


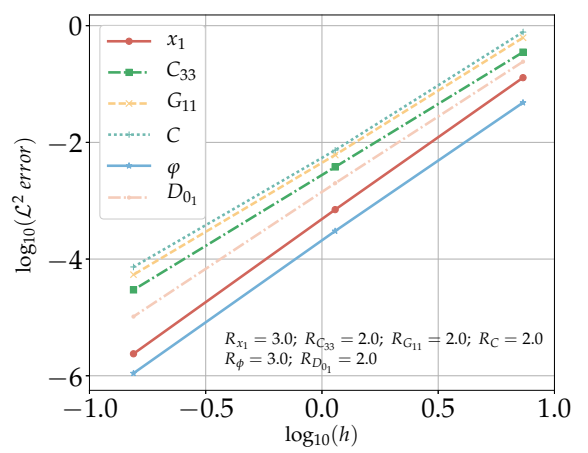

(a)

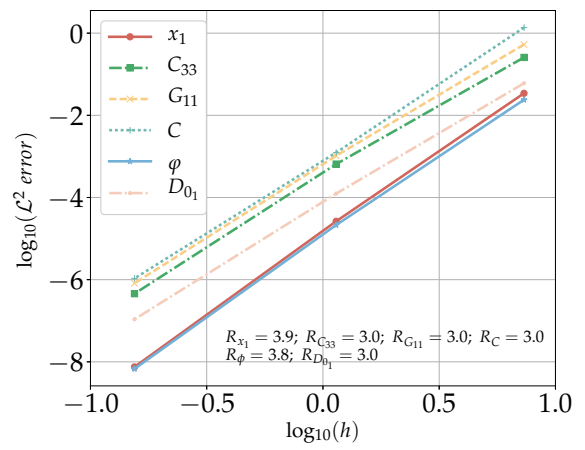

(c)

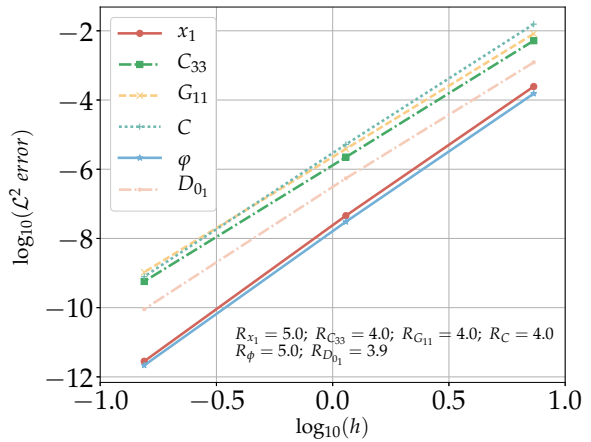

(e)

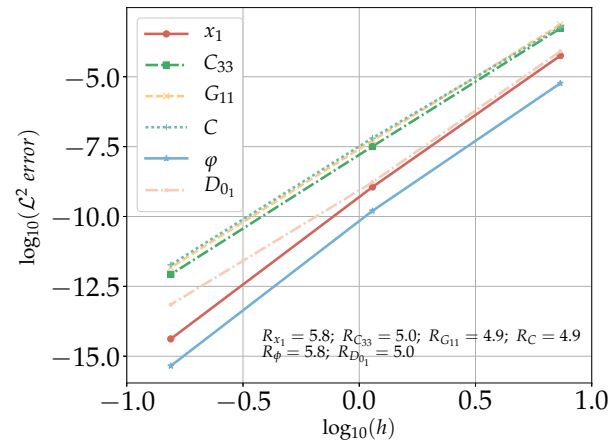

(g)

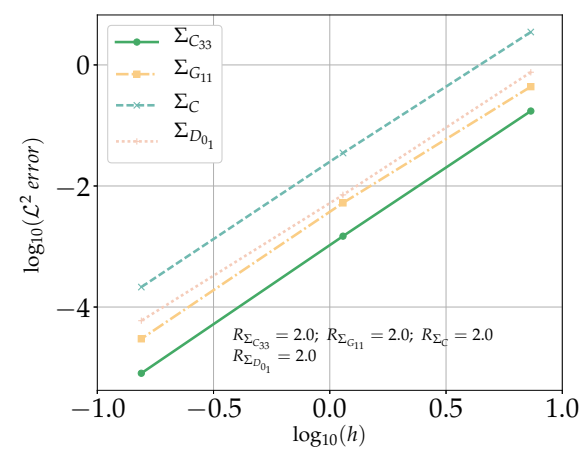

(b)

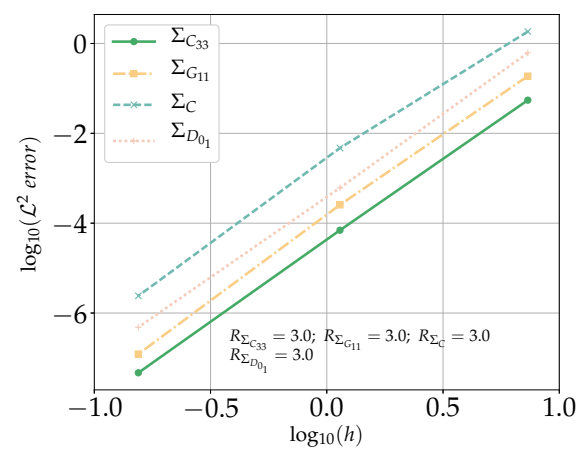

(d)

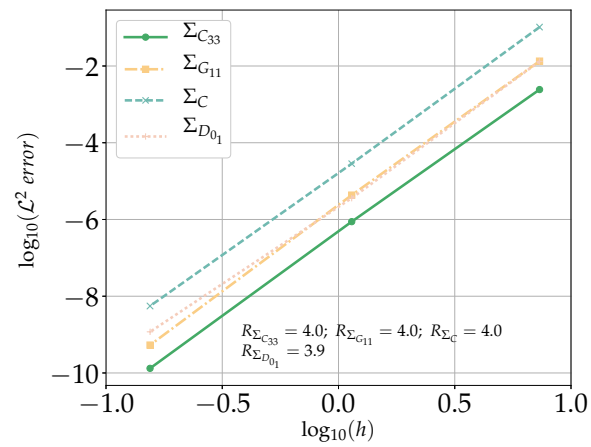

(f)

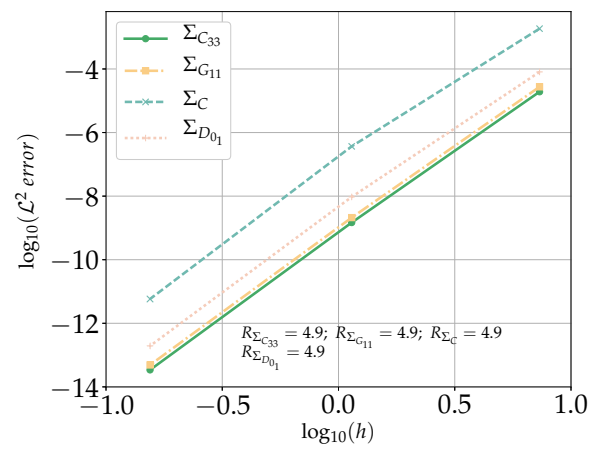

(h)

Figure 6: $h$ convergence of $\mathcal{L}^{2}(V)$ norm of the error for different kinematic and kinetic variables, (a) \& (b) $p=2$, (c) \& (d) $p=3$, (e) \& (f) $p=4$ and (g) \& (h) $p=5$. $R_{\zeta}$ indicates the rate of convergence of quantity $\zeta$. 


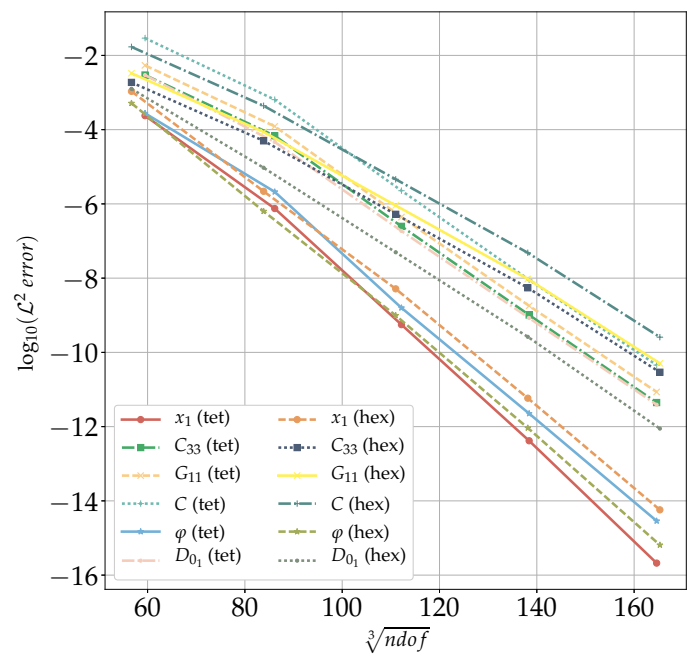

(a)

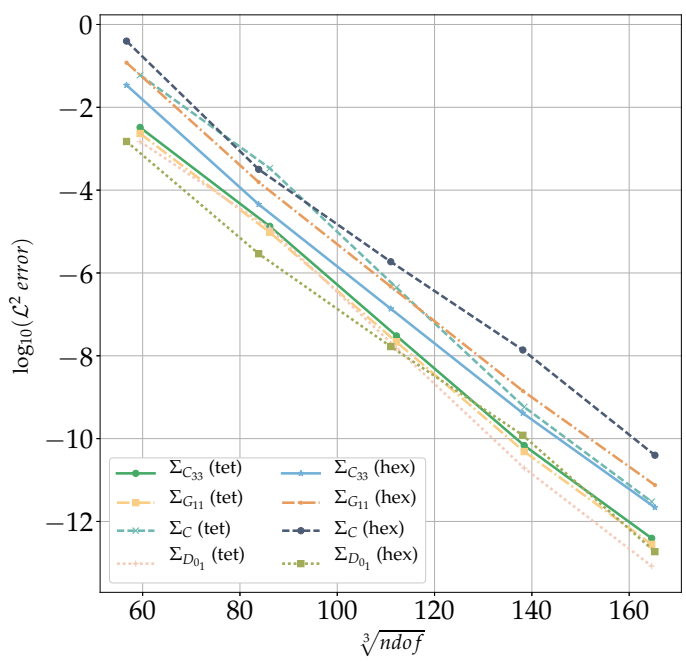

(b)

Figure 7: $p$ convergence of $\mathcal{L}^{2}(V)$ norm of the error for different kinematic and kinetic variables for mesh with 9220 elements.

\begin{tabular}{|l|l|l|l|l|}
\hline$\mu_{1}(\mathrm{~Pa})$ & $\mu_{2}(\mathrm{~Pa})$ & $\lambda(\mathrm{Pa})$ & $\varepsilon_{1}\left(\mathrm{~N} / \mathrm{V}^{2}\right)$ & $\varepsilon_{2}\left(\mathrm{~N} / \mathrm{V}^{2}\right)$ \\
\hline $1 e 5$ & $1 e 5$ & $5 e 5$ & $\varepsilon_{0}$ & $5 \varepsilon_{0}$ \\
\hline
\end{tabular}

Table 5: Material parameters for (41) with the vacuum permittivity $\varepsilon_{0}=8.85418781 \times 10^{-12}\left(\mathrm{~N} / \mathrm{V}^{2}\right)$

and the geometry and the tetrahedral and hexahedral meshes are shown in Figure 8 and Figure 9, respectively. A constant electric voltage of $2 \times 10^{8} \mathrm{~V} / \mathrm{m}$ is applied through the whole thickness

\begin{tabular}{|c|c|c|c|c|c|c|c|c|}
\hline \multirow[b]{2}{*}{$p$} & \multicolumn{4}{|c|}{ Tetrahedral } & \multicolumn{4}{|c|}{ Hexahedral } \\
\hline & DoFs & $Q_{1}$ & $Q_{2}$ & $Q_{3}$ & DoFs & $Q_{1}$ & $Q_{2}$ & $Q_{3}$ \\
\hline$p / q=2$ & $340 \times 4$ & 0.982 & 0.965 & 0.948 & $363 \times 4$ & 0.980 & 0.961 & 0.942 \\
\hline$p / q=3$ & $938 \times 4$ & 0.990 & 0.980 & 0.971 & $1012 \times 4$ & 0.982 & 0.966 & 0.951 \\
\hline$p / q=4$ & $2021 \times 4$ & 0.986 & 0.973 & 0.960 & $2165 \times 4$ & 0.981 & 0.963 & 0.947 \\
\hline$p / q=5$ & $3681 \times 4$ & 0.990 & 0.980 & 0.970 & $3966 \times 4$ & 0.979 & 0.959 & 0.941 \\
\hline$p / q=6$ & $6097 \times 4$ & 0.990 & 0.979 & 0.969 & $6559 \times 4$ & 0.977 & 0.955 & 0.935 \\
\hline
\end{tabular}

Table 6: Distortion quality of high order curvilinear tetrahedral and hexahedral meshes

and symmetric mechanical Dirichlet boundary conditions are imposed on the outer boundaries of the plate. This induces stretching of the electromechanical plate and as a result of the outer boundaries being fixed the plate is thickened in the region away from the hole and shrunk in thickness in the vicinity of the hole. Figure 10 shows the quadratic convergence of NewtonRaphson for the last load increment for all polynomial degrees on curvilinear tetrahedral and hexahedral meshes, respectively. It is worth mentioning that, since the planar and curvilinear meshes do not possess the same volume, a systematic study of the difference in error norms of quantities is not feasible. However, since both planar and curved meshes share the same $(p / q=1)$ vertices, a comparison of certain quantities of interest at these vertices can be carried out. Notice that since the node is shared between neighbouring elements appropriate stress recovery is required. This will lead to some oscillatory results in stresses (c.f. subsection 4.4 for further investigation in this regard). In the current setting, the order of quadrature rule to integrate stresses have been purposefully increased to $2(p+1)$ to remove these oscillations as far as feasible. A representation of stress concentration for the plate with circular hole is shown 


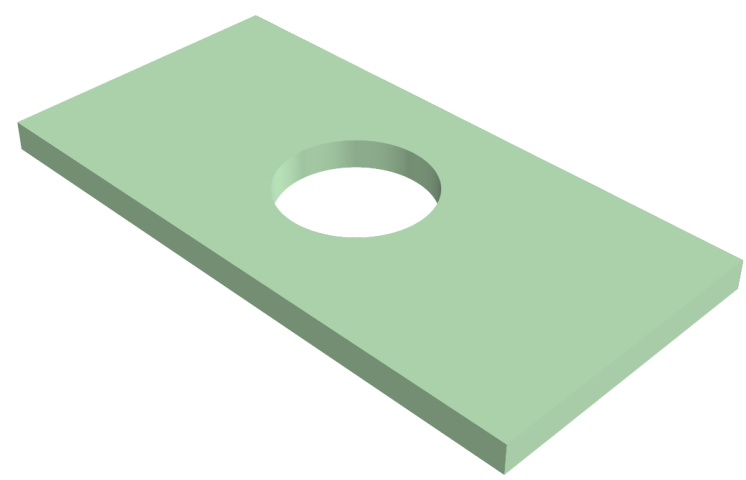

Figure 8: CAD representation of plate with circular hole with dimensions $10 \times 20 \times 2 \mathrm{~m}^{3}$. The circular hole with a radius of $5 \mathrm{~m}$ is centred at $[0,0,0]^{T}$

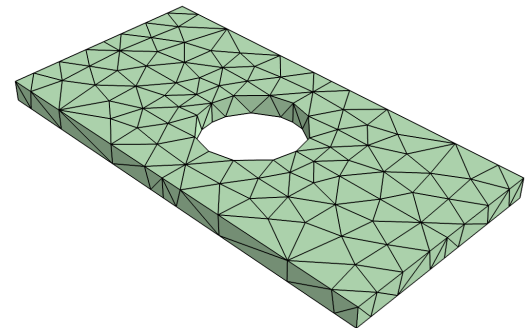

(a)

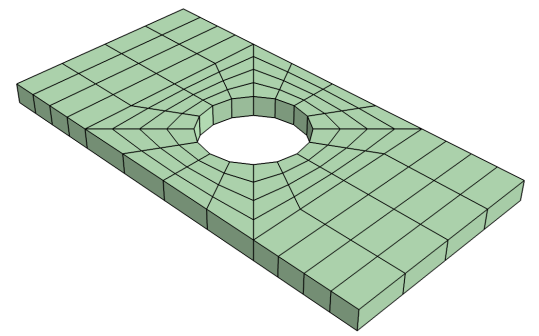

(c)

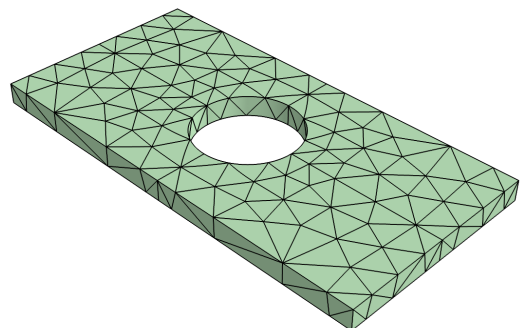

(b)

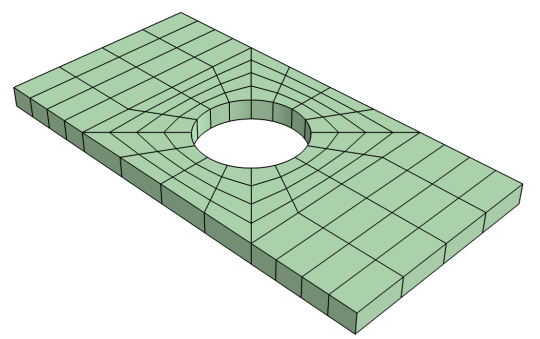

(d)

Figure 9: Curvilinear meshes used for comparison a) $p=3$ planar tetrahedra, b) $p=3$ curvilinear tetrahedra, c) $p=3$ planar hexahedra and, d) $p=3$ curvilinear hexahedra

figuratively in Figure 11 only for $p / q=5$ meshes, for the final deformed configuration. It is evident from the figures that as opposed to the meshes with planar faces/edges, curvilinear meshes perform much better in reporting a smoother representation of the stress near the circular region. A similar conclusion is drawn across all polynomial degrees for tetrahedral and hexahedral elements (not reported, for the purpose of brevity). For instance, the maximum hydrostatic pressure at the tip of the circular hole within the plate located at $[5,20,2]^{T}$ is $p_{\text {hyd }}=-4.982 \times 10^{4} \mathrm{~Pa}$ for the hexahedral mesh with planar faces/edges, whereas only $p_{\text {hyd }}=-0.104 \mathrm{~Pa}$ for curvilinear hexahedral mesh. This clearly confirms that the appearance of non-physical stress concentrations can be significantly reduced through curvilinear meshes accurately representing the geometry. Similar behaviour has also been reported by the computational fluid dynamics community, where an inaccurate geometric representation leads to non-physical entropy production [58, 57]. In line with the theme of this work, the aim of this study has been to assess and report the performance of curvilinear meshes in comparison to high order meshes with planar facets purely based on 


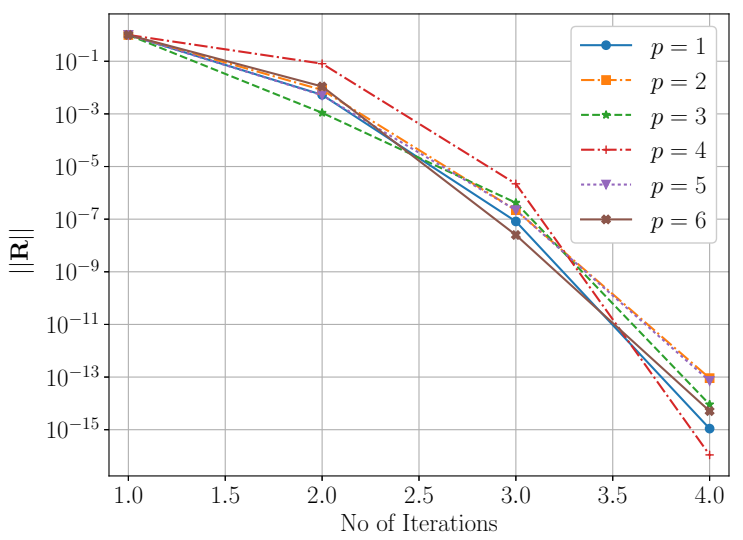

(a)

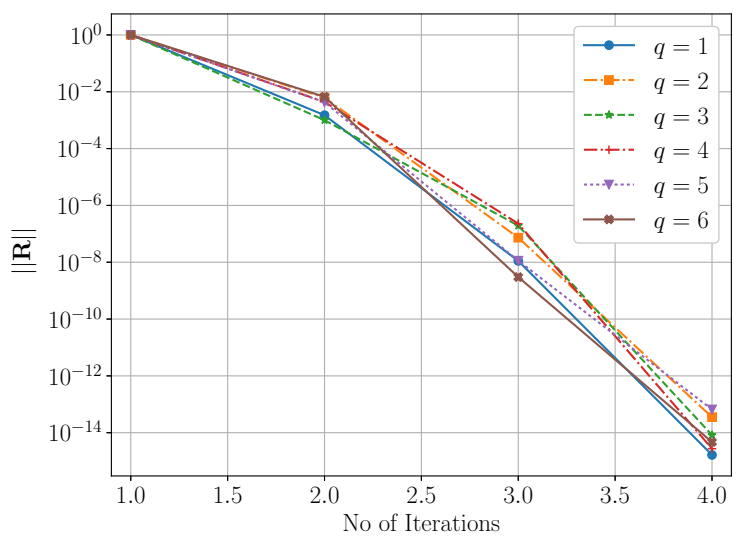

(b)

Figure 10: Quadratic convergence of Newton-Raphson on curivilinear a ) tetrahedral mesh and, b) hexahedral mesh

geometrical enhancements.

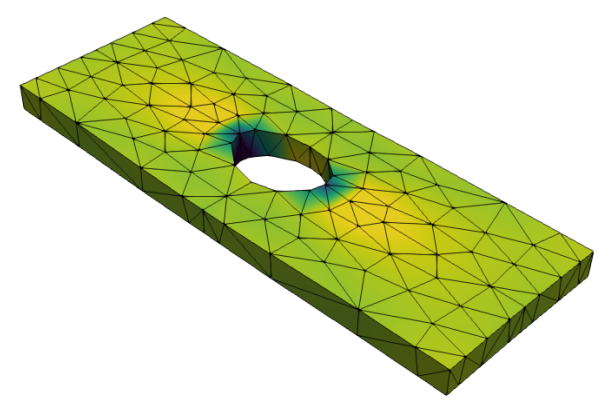

(a)

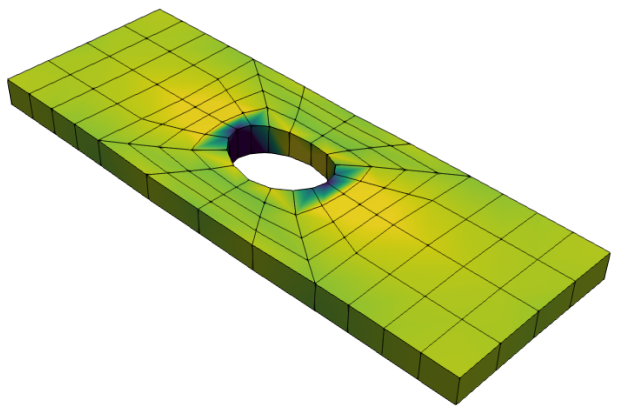

(c)

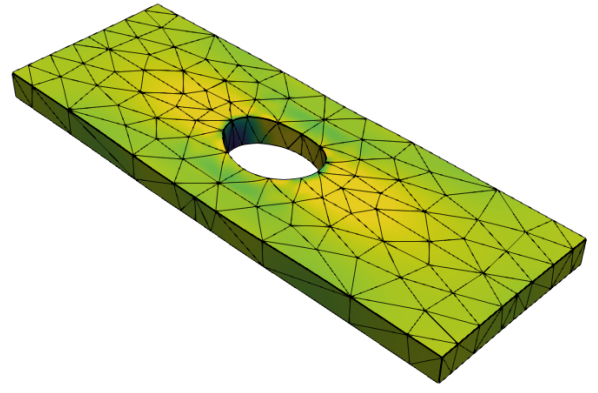

(b)

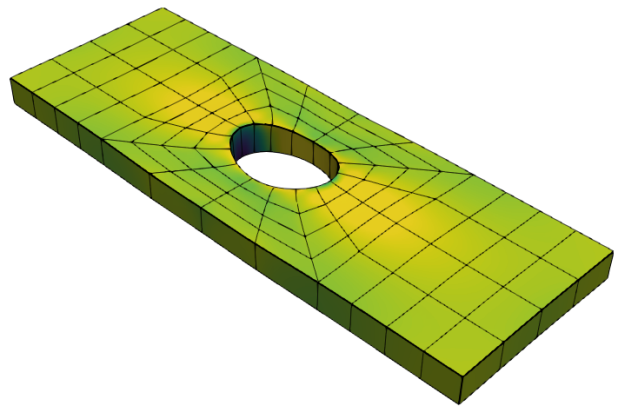

(d)

$p_{\text {hyd }}$

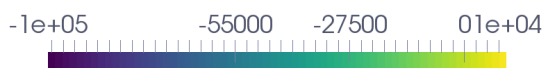

Figure 11: Evolution of voltage induced hydrostatic pressure in a plate with circular hole, a) planar $p=5$ mesh, b) curved $p=5$ mesh, c) planar $q=5$ mesh and, d) curved $q=5$ mesh

\subsection{Linearised electrostriction through the staggered approach}

The objective of this example is to study the performance and the range of applicability of the staggered approach for small strain actuation and energy harvesting problems. To this end, 
two actuation problems are considered, based on two material models. For the first problem, the material model used is that of (41) with material constant given by (5). This problem is in particular a simple one due to the fact that electric field is a linear function of electric displacement and hence chosen for the purpose of benchmarking the staggered scheme. For the second problem, the following stabilised convex multi-variable energy functional is considered [3]

$$
\begin{array}{r}
W_{e l, 2}=\mu_{1} I_{\boldsymbol{C}}+\mu_{2} I_{\boldsymbol{G}}-\left(2 \mu_{1}+4 \mu_{2}+12 \mu_{e}\right) \ln \sqrt{C}+\frac{\lambda}{2}(\sqrt{C}-1)^{2}+\frac{1}{2 \varepsilon_{1}} I I_{\boldsymbol{D}_{0}}+\frac{1}{2 \varepsilon_{2} \sqrt{C}} I I_{\boldsymbol{d}} \\
+\mu_{e}\left(I_{\boldsymbol{C}}^{2}+\frac{2}{\mu_{e} \varepsilon_{e}} I_{\boldsymbol{C}} I I_{\boldsymbol{d}}+\frac{1}{\mu_{e}^{2} \varepsilon_{e}^{2}} I I_{\boldsymbol{d}}^{2}\right)
\end{array}
$$

with material properties listed in Table 7 . In this model the electric field and electric displacement

\begin{tabular}{|l|l|l|l|l|l|l|}
\hline$\mu_{1}(\mathrm{~Pa})$ & $\mu_{2}(\mathrm{~Pa})$ & $\mu_{e}(\mathrm{~Pa})$ & $\lambda(\mathrm{Pa})$ & $\varepsilon_{1}^{-1}\left(\mathrm{~V}^{2} / \mathrm{N}\right)$ & $\varepsilon_{2}\left(\mathrm{~N} / \mathrm{V}^{2}\right)$ & $\varepsilon_{2}\left(\mathrm{~N} / \mathrm{V}^{2}\right)$ \\
\hline $2.3 e 4$ & $1.15 e 5$ & 800 & $8 e 5$ & 0 & $4.5 \varepsilon_{0}$ & $1050 \varepsilon_{0}$ \\
\hline
\end{tabular}

Table 7: Material parameters for $(42)$ with the vacuum permittivity $\varepsilon_{0}=8.85418781 \times 10^{-12}\left(\mathrm{~N} / \mathrm{V}^{2}\right)$

are coupled implicitly and hence this model tests the true performance of the staggered approach. The computational meshes considered are shown in Figure 12 together with the CAD geometry. The mesh has 3819 elements and for an interpolation degree $p=3$ there are 47317 points in the mesh corresponding to a total of 189268 degrees of freedom. To study the performance of the

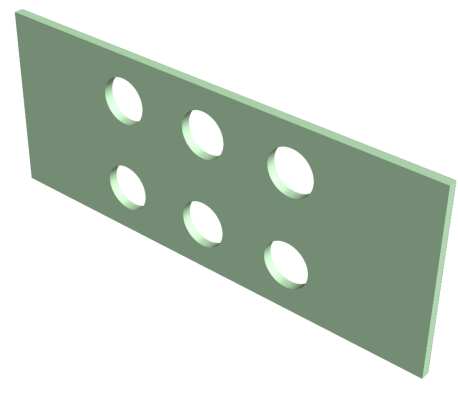

(a)

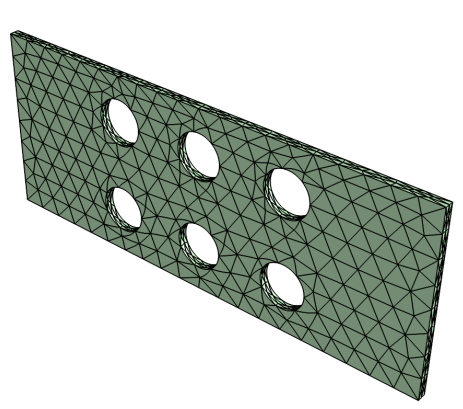

(b)

Figure 12: Electromechanical plate with holes a) CAD geometry and, b) $p=4$ curvilinear mesh

staggered scheme the error incurred in staggered scheme is quantified relative to (fully nonlinear) monolithic approach as

$$
\|e\|_{\mathcal{L}^{2}}^{\text {staggered }}=\left[\frac{\left\|\boldsymbol{\eta}_{\text {monolithic }}-\boldsymbol{\eta}_{\text {staggered }}\right\|}{\left\|\boldsymbol{\eta}_{\text {monolithic }}\right\|}\right]^{\frac{1}{2}}
$$

Typically, the range of applicability of the staggered electrostrictive scheme can be determined by successively increasing the electric voltage and observing the error produced by the scheme [25]. In the current setting, the following non-dimensional quantity is chosen to quantify the 
increase in electric voltage

$$
\mathcal{W}=\Phi /\left(H \sqrt{\frac{\mu}{\varepsilon}}\right),
$$

where $\Phi$ denotes the applied potential and $H$ represents the plate thickness. For the ideal dielectric model $\mu$ and $\varepsilon$ can be related to material parameters as

$$
\mu=2\left(\mu_{1}+\mu_{2}\right) ; \quad \frac{1}{\varepsilon}=\frac{1}{\varepsilon_{1}}+\frac{1}{\varepsilon_{2}} .
$$

Similarly, for the stabilised material described in (42), $\mu$ and $\varepsilon$ can be found as

$$
\mu=2\left(\mu_{1}+2 \mu_{2}+6 \mu_{e}\right) ; \quad \frac{1}{\varepsilon}=\frac{1}{\varepsilon_{1}}+\frac{1}{\varepsilon_{2}}+\frac{12}{\varepsilon_{e}} .
$$

The plate is clamped at the two ends along the length and the electric voltage is applied through the thickness of the plate such that $\mathcal{W}$ increases from 0.1 to 1.0. Figure 13 shows the relative $\mathcal{L}^{2}$ norm of the staggered approach as function of number of increments for different values of $\mathcal{W}$. As can be observed, certainly as the $\mathcal{W}$ and hence the voltage induced deformation increases, the error incurred in staggered approach increases. The convergence of the staggered approach is approximately linear for both models. Interestingly, even under the application of extremely high voltage, the results of the staggered scheme seem to converge to the results of the monolithic approach. However, opting for such high number of increments may not be computationally practical. For engineering accuracy and large scale problems, the staggered scheme pays off as it only involves an iterative solution of the Gauss's law for the scalar electric potential.

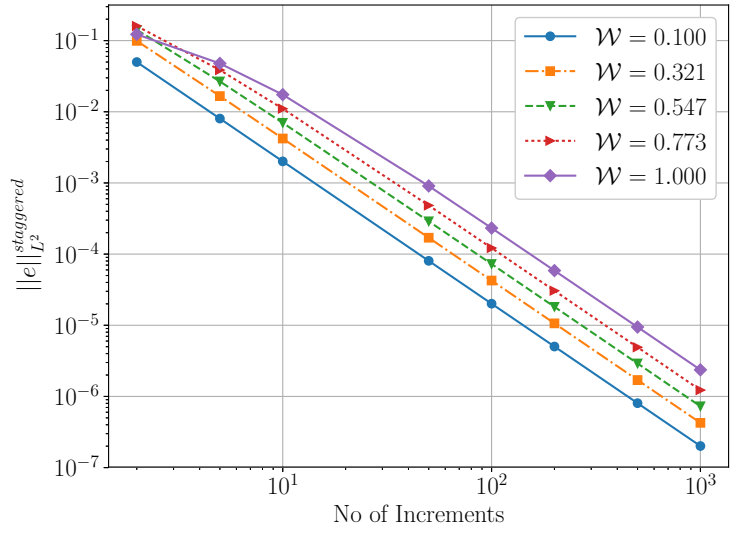

(a)

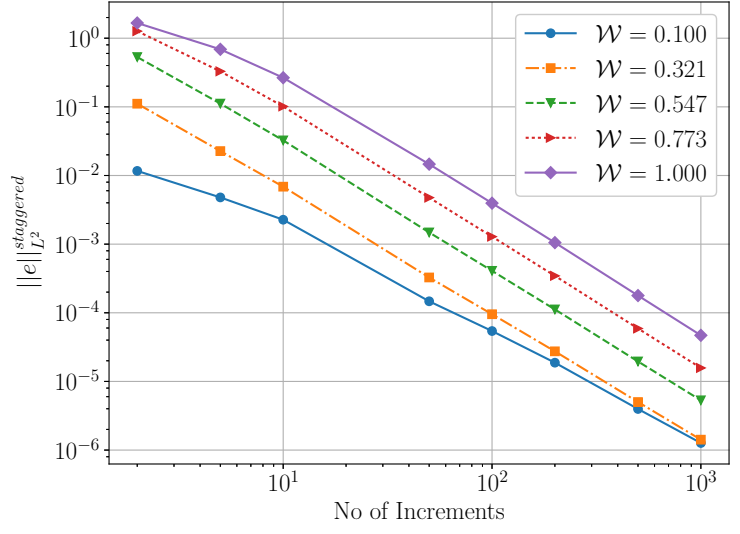

(b)

Figure 13: Convergence of the staggered scheme with respect to the monolithic solver as a function of increase in applied voltage quantified by $\mathcal{W}$ as defined in (44)

Finally, Figure 14 shows the amount of voltage induced strains in the plate. Notice that despite large displacements, the strains are still small in the context of dielectric elastomers. 


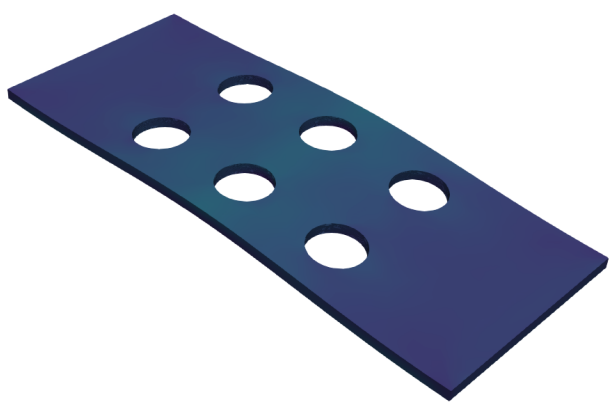

(a)

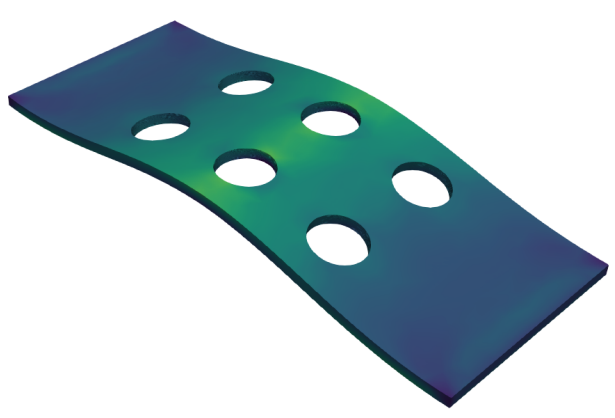

(c)

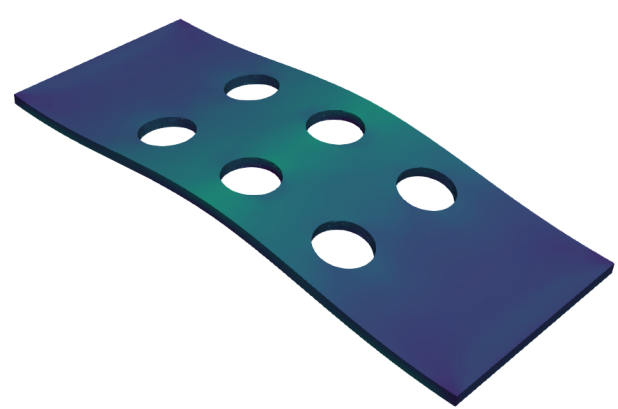

(b)

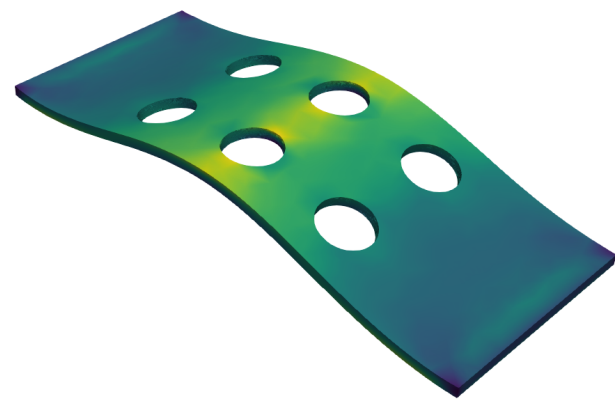

(d)

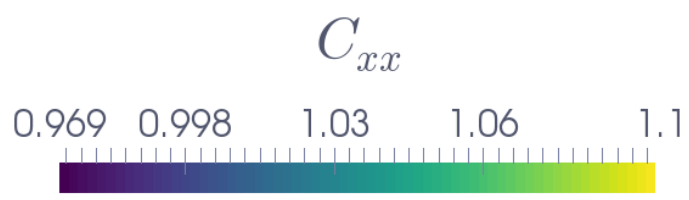

Figure 14: Voltage induced strains $\boldsymbol{C}_{x x}$ in electromechanical plate

\subsection{Comparison of higher order displacement potential based formulation with 11 field mixed formulation for electro-elasticity}

The objective of the examples presented in this section is to compare the performance of the displacement-potential formulation (DPF) discretised using high order tetrahedral and hexahedral finite elements with the 11 field mixed Hu-Washizu variational formulation (MWF) (based on the set $\left.\left\{\boldsymbol{x}, \boldsymbol{F}, \boldsymbol{H}, J, \varphi, \boldsymbol{d}, \boldsymbol{\Sigma}_{\boldsymbol{F}}, \boldsymbol{\Sigma}_{\boldsymbol{H}}, \Sigma_{J}, \boldsymbol{\Sigma}_{\boldsymbol{D}_{0}}, \boldsymbol{\Sigma}_{\boldsymbol{d}}\right\}\right)$ for electro-elasticity presented in [1, 2, 16], on curvilinear meshes. In particular, MWF is an extension of the mixed formulations to polyconvex elasticity presented in $[41,22,62]$. While for the DPF formulation, an equal order interpolation for all the variables $(\boldsymbol{x}, \varphi)$ is used (for tetrahedra and hexahedra), the MWF formulation utilises a quadratic interpolation for $\boldsymbol{x}$ and $\varphi$, linear discontinuous interpolation for $\boldsymbol{F}, \boldsymbol{H}, J, \boldsymbol{d}, \boldsymbol{\Sigma}_{\boldsymbol{F}}, \boldsymbol{\Sigma}_{\boldsymbol{H}}, \boldsymbol{\Sigma}_{\boldsymbol{D}_{0}}, \boldsymbol{\Sigma}_{\boldsymbol{d}}$ and a piecewise constant interpolation for $J$ and $\Sigma_{J}$ and is primarily developed for 10-noded tetrahedra [2]. These formulations are compared against with an eye on two fundamental issues pertaining to the modelling of large deformation large electric field electromechanics namely, shear-locking and volumetric locking. To this end, three dielectric cantilever patches with aspect ratio $\{10,100,1000\}$ are considered, as shown in Figure 15. The problem is analysed under compressible and nearly incompressible scenarios with Poisson's ratio 0.45 and 0.499 respectively. A series of curvilinear meshes are then produced for $p=\{2,3,4,5\}$ and $q=\{2,3,4,5\}$ for each aspect ratio. In addition, three $h$-refinement levels are considered 
using a coarse, a medium and a fine description for each aspect ratio and each polynomial degree. An attempt is made to keep the total number of nodes in the computational mesh for every polynomial degree within the same range (within $5 \%$ difference). The necessary information regarding the quality of the curvilinear meshes are listed in Table 8 and Table 9 , for tetrahedral meshes and hexahedral meshes, respectively. Note that, for a given $p$ and $h$-refinement level, the size of the computational mesh is the same for all aspect ratios, in that, to obtain different aspect ratios, the mesh is appropriately scaled in the thickness direction. This certainly leads to extremely stretched elements but does not change the distortion of quality of the mesh as far as curvilinear mesh quality measures are concerned.

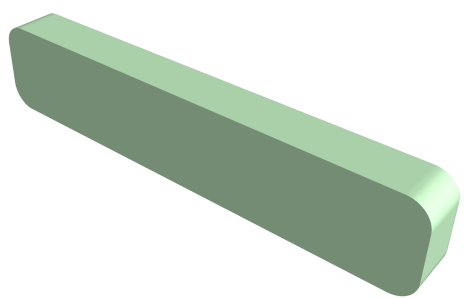

(a)

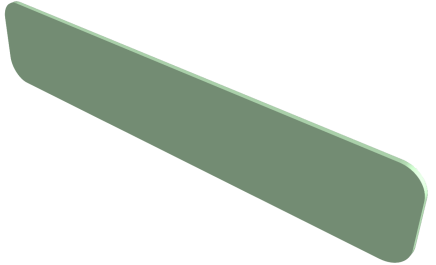

(b)

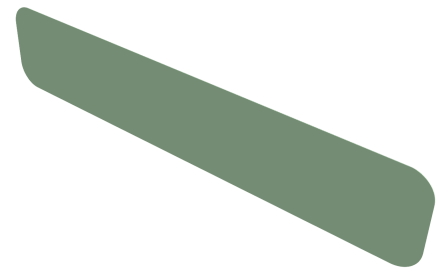

(c)

Figure 15: Geometry of the patch for three different aspect ratios a) 10, b) 100 and, c) 1000

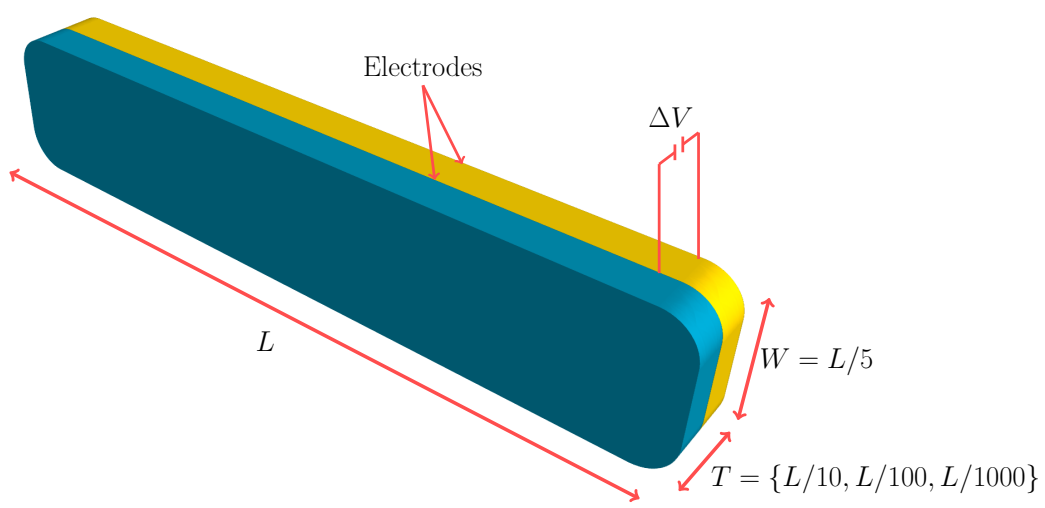

Figure 16: Description of the boundary conditions

\begin{tabular}{|c|c|c|c|c|c|c|c|c|c|c|c|c|}
\hline \multirow[b]{2}{*}{$p$} & \multicolumn{4}{|c|}{ Coarse } & \multicolumn{4}{|c|}{ Medium } & \multicolumn{4}{|c|}{ Fine } \\
\hline & DoFs (N. Elements) & $Q_{1}$ & $Q_{2}$ & $Q_{3}$ & DoFs (N. Elements) & $Q_{1}$ & $Q_{2}$ & $Q_{3}$ & DoFs (N. Elements) & $Q_{1}$ & $Q_{2}$ & $Q_{3}$ \\
\hline$p=2$ & $2842 \times 4(1652)$ & 0.971 & 0.943 & 0.909 & $4686 \times 4(2730)$ & 0.965 & 0.936 & 0.911 & $9916 \times 4(5910)$ & 0.980 & 0.959 & 0.938 \\
\hline$p=3$ & $2859 \times 4(396)$ & 0.911 & 0.784 & 0.586 & $4656 \times 4(710)$ & 0.925 & 0.837 & 0.734 & $9838 \times 4(1866)$ & 0.952 & 0.898 & 0.836 \\
\hline$p=4$ & $2835 \times 4(126)$ & 0.852 & 0.690 & 0.500 & $4693 \times 4(326)$ & 0.872 & 0.733 & 0.611 & $9819 \times 4(698)$ & 0.945 & 0.886 & 0.819 \\
\hline$p=5$ & $2856 \times 4(82)$ & 0.873 & 0.758 & 0.651 & $4651 \times 4(172)$ & 0.952 & 0.908 & 0.866 & $9889 \times 4(396)$ & 0.944 & 0.890 & 0.840 \\
\hline
\end{tabular}

Table 8: Distortion quality of high order curvilinear tetrahedral meshes

For comparison an ideal dielectric elastomer is considered with the following convex multivariable internal energy description

$$
W_{e l, 3}=\mu_{1} I_{\boldsymbol{C}}+\mu_{2} I_{\boldsymbol{G}}-2\left(\mu_{1}+\mu_{2}\right) \ln \sqrt{C}+\frac{\lambda}{2}(\sqrt{C}-1)^{2}+\frac{1}{2 \varepsilon_{1}} I I_{\boldsymbol{d}}
$$

with the material parameters as given in Table 10. 


\begin{tabular}{|l|l|l|l|l|l|l|l|l|l|l|l|l|}
\hline $\boldsymbol{p}$ & \multicolumn{9}{c|}{ Coarse } & \multicolumn{4}{c|}{} & \multicolumn{2}{c|}{ Fedium } \\
$\boldsymbol{p}$ & DoFs (N. Elements) & $Q_{1}$ & $Q_{2}$ & $Q_{3}$ & DoFs (N. Elements) & $Q_{1}$ & $Q_{2}$ & $Q_{3}$ & DoFs (N. Elements) & $Q_{1}$ & $Q_{2}$ & $Q_{3}$ \\
\hline$q=2$ & $2885 \times 4(244)$ & 0.972 & 0.987 & 0.957 & $4665 \times 4(472)$ & 0.986 & 0.970 & 0.953 & $9805 \times 4(928)$ \\
\hline$q=3$ & $2853 \times 4(76)$ & 0.959 & 0.913 & 0.863 & $4605 \times 4(148)$ & 0.979 & 0.957 & 0.935 & $9805 \times 4(292)$ & 0.990 & 0.980 & 0.969 \\
\hline$q=4$ & $2873 \times 4(32)$ & 0.818 & 0.618 & 0.361 & $4650 \times 4(68)$ & 0.950 & 0.897 & 0.842 & $9793 \times 4(124)$ & 0.979 & 0.958 & 0.935 \\
\hline$q=5$ & $2756 \times 4(16)$ & 0.822 & 0.625 & 0.369 & $4700 \times 4(32)$ & 0.834 & 0.656 & 0.401 & $9801 \times 4(64)$ & 0.950 & 0.898 & 0.842 \\
\hline
\end{tabular}

Table 9: Distortion quality of high order curvilinear hexahedral meshes

\begin{tabular}{|l|l|l|l|l|}
\hline Parameters & $\mu_{1}(\mathrm{~Pa})$ & $\mu_{2}(\mathrm{~Pa})$ & $\lambda(\mathrm{Pa})$ & $\varepsilon_{1}\left(\mathrm{~N} / \mathrm{V}^{2}\right)$ \\
\hline Values (for $\nu=0.45)$ & $1 e 5$ & 0 & $9 e 5$ & $4 \varepsilon_{0}$ \\
\hline Values (for $\nu=0.499)$ & $1 e 5$ & 0 & $4.99 e 7$ & $4 \varepsilon_{0}$ \\
\hline
\end{tabular}

Table 10: Material parameters for (47) with the vacuum permittivity $\varepsilon_{0}=8.85418781 \times 10^{-12}\left(\mathrm{~N} / \mathrm{V}^{2}\right)$

In order to be able to apply appropriate boundary conditions, it is made sure that all computational meshes have at least two elements across the thickness. For every aspect ratio, the patch is clamped at $[0, X, Y]^{T}$ and a constant electric voltage is applied across the half thickness $T / 2$ (where $T$ represents the patch thickness), as shown in Figure 16. It is certainly not feasible to apply the same amount of electric voltage while varying the patch aspect ratio and hence Table 11 lists the Dirichlet boundary conditions considered for the three aspect ratios.

\begin{tabular}{|l|l|l|l|}
\hline Aspect Ratio & 10 & 100 & 1000 \\
\hline Electric Voltage $(V / m)$ & $1 e 7$ & $7.5 e 4$ & $1.8 e 2$ \\
\hline
\end{tabular}

Table 11: Applied electric voltage as Dirichlet boundary condition for three aspect ratios

In order to compare different kinematic and kinetic quantities of interest for different formulations, two different physical points in the patches are considered namely, $A$ - the node located at $[L, W / 2, T / 2]^{T}$, and $B$ - the point interior to the computational mesh $[0.98 L, 0.49 W, 0.49 T]^{T}$ (c.f. Figure 16).

Table 12 and Table 13 compare the results obtained for different kinematics and kinetics quantities with displacement potential formulation using high order tetrahedral and hexahedral elements with the 11 field mixed formulations, for aspect ratio 10 and Poisson's ratio 0.45 and 0.499, respectively. For this comparison, the problem is solved in 50 load increments and the comparison is performed at increment 12 , which corresponds to the onset of nonlinear deformation. The first observation from the tables is that the DPF based discretisations have an asymptotically upper bound convergence while the MWF based results have an asymptotically lower bound convergence. This can be observed studying the convergence of a given quantity for a fixed polynomial degree and successive $h$-refinements. This convergence pattern is more pronounced in the case of MWF. Consequently, for a fixed polynomial degree, it can be observed that MWF has a consistent $2 \%-5 \%$ higher displacements and strains estimate rate.

Regarding the stresses, as can be seen from the $\sigma_{x z}$ results, the differences between the formulations are significant. While the results for the MWF show a convergence pattern, the results of the DPF do not seem to converge as coherently, with some clear pressure oscillations (as $p / q$ and $h$-refinements are carried out). The $\sigma_{z z}$ and hydrostatic pressure still do not seem to be affected, primarily due to the fact that these components have a much higher electro-mechanical stress contribution coming from the electric displacement (which is the applied Dirichlet boundary condition).

The conclusion drawn from the above set of results is that, for thick electromechanical beams, both high order DPF and MWF implementations capture the physical behaviour of the system 
accurately without showing any signs of shear or volumetric lockings. Importantly, this signifies that high order discretisations can capture nearly incompressible scenarios reliably [59, 60, 61]. The oscillations in stresses is an expected phenomenon for DPF implementations [2, 62, 41] which seems to persist even with polynomial enrichment. Unlike the problems reported in subsection 4.1, the convergence rate of stresses here can not be ascertained, since the polynomial enrichment here, comes with the trade-off of coarsening the computational mesh to keep the problem size the same. In this regard, MWF implementation maintains a consistent accuracy by virtue of explicitly solving for kinetic variables.

Note that so far, only the aspect ratio of the patch has been considered and no remarks have been made regarding the aspect ratio of the elements in the mesh. It is important to mention that high order elements are at a severe disadvantage here compared to their low-order or MWF counterparts, in that, polynomial enrichment leading to coarsening of the mesh, also inherently implies dramatic stretching for high order elements. It is certainly not feasible to control the aspect ratio of the patch and the aspect ratio of the elements at the same time, while also having to keep the size of the computational mesh the same across all polynomial enrichments. This also signifies that, for $p=q$, the computational meshes do not necessarily exhibit the same elemental aspect ratios. It is still astounding to observe that, high order elements perform fairly well regardless.

\begin{tabular}{|c|c|c|c|c|c|c|c|c|c|c|}
\hline Quantity & Refinement & $p=2$ & $p=3$ & $p=4$ & $p=5$ & $q=2$ & $q=3$ & $q=4$ & $q=5$ & 11Mixed \\
\hline \multirow{3}{*}{$u_{x}^{A}(m)$} & Coarse & 0.15 & 0.1506 & 0.1502 & 0.1505 & 0.1505 & 0.1506 & 0.1506 & 0.1506 & 0.1595 \\
\hline & Medium & 0.1505 & 0.1503 & 0.1505 & 0.1506 & 0.1506 & 0.1506 & 0.1507 & 0.1506 & 0.1542 \\
\hline & Fine & 0.1505 & 0.1508 & 0.1505 & 0.1506 & 0.1506 & 0.1506 & 0.1507 & 0.1506 & 0.1535 \\
\hline \multirow{3}{*}{$u_{z}^{A}(m)$} & Coarse & 0.9572 & 0.9555 & 0.9565 & 0.9551 & 0.958 & 0.9597 & 0.9598 & 0.9603 & 1.049 \\
\hline & Medium & 0.9566 & 0.9576 & 0.9603 & 0.9596 & 0.9583 & 0.96 & 0.9604 & 0.9603 & 1.0022 \\
\hline & Fine & 0.9571 & 0.96 & 0.9597 & 0.9594 & 0.9585 & 0.96 & 0.9604 & 0.9603 & 0.9948 \\
\hline \multirow{3}{*}{$F_{x z}^{A}$} & Coarse & -0.0176 & -0.0186 & -0.018 & -0.0184 & -0.0178 & -0.0184 & -0.0183 & -0.0183 & -0.0199 \\
\hline & Medium & -0.0183 & -0.0181 & -0.018 & -0.0183 & -0.0178 & -0.0185 & -0.0183 & -0.0183 & -0.0191 \\
\hline & Fine & -0.0183 & -0.0186 & -0.0178 & -0.0184 & -0.0178 & -0.0185 & -0.0183 & -0.0183 & -0.0191 \\
\hline \multirow{3}{*}{$H_{z x}^{A}$} & Coarse & -0.0184 & -0.0184 & -0.0182 & -0.0186 & -0.0181 & -0.0184 & -0.0183 & -0.0183 & -0.0198 \\
\hline & Medium & -0.0184 & -0.0181 & -0.0183 & -0.0182 & -0.018 & -0.0185 & -0.0184 & -0.0183 & -0.019 \\
\hline & Fine & -0.0184 & -0.0181 & -0.0186 & -0.0184 & -0.018 & -0.0185 & -0.0184 & -0.0183 & -0.0192 \\
\hline \multirow{3}{*}{$\sigma_{x z}^{B}(\mathrm{~Pa})$} & Coarse & 68.817 & -34.4955 & 8.4401 & 2.5424 & 20.9594 & -16.3045 & -4.2272 & -8.6977 & -24.0193 \\
\hline & Medium & 1.5908 & -10.4219 & 16.6441 & -17.0173 & 11.9318 & -4.436 & -4.8418 & -8.9388 & -15.3804 \\
\hline & Fine & -3.9871 & -63.5881 & 19.221 & -15.5529 & 7.1833 & -4.433 & -4.8497 & -8.9573 & -4.7419 \\
\hline \multirow{3}{*}{$\sigma_{z z}^{B}(\mathrm{~Pa})$} & Coarse & 228.8202 & 242.9458 & 167.4105 & 225.4981 & 227.3906 & 208.8909 & 220.5269 & 233.9105 & 262.6845 \\
\hline & Medium & 226.3231 & 304.8385 & 190.6815 & 232.3852 & 228.7893 & 202.9177 & 228.5124 & 234.1146 & 262.0383 \\
\hline & Fine & 226.7235 & 228.4325 & 192.3997 & 227.9372 & 229.1137 & 202.8566 & 228.503 & 234.1104 & 233.9687 \\
\hline \multirow{3}{*}{$p_{h y d}^{B}(\mathrm{~Pa})$} & Coarse & 258.7676 & 262.7815 & 228.257 & 256.8133 & 249.9223 & 258.8183 & 253.0553 & 260.2188 & 283.1432 \\
\hline & Medium & 256.2272 & 239.7233 & 239.8217 & 256.7475 & 249.6441 & 259.3416 & 254.3613 & 260.1902 & 279.219 \\
\hline & Fine & 257.2179 & 258.3805 & 255.9474 & 256.809 & 249.4617 & 259.3333 & 254.3594 & 260.1998 & 229.3169 \\
\hline
\end{tabular}

Table 12: Comparison of high order displacement potential and 11 field mixed formulations for different kinematic and kinetic measures, for aspect ratio 10 and Poisson's ratio 0.45

Turning the attention to thinner beams, the results for the patch with the aspect ratio 100 are analogous to those with the aspect ratio 10 and offer no further insight, and are hence omitted for the purpose of brevity. The same analysis is then carried out with extremely thin beams with aspect ratio 1000 featuring highly stretched elements. The corresponding loading from Table 11 is applied. Table 14 and Table 15 compare the results obtained for different kinematics and kinetics quantities with displacement potential formulation using high order tetrahedral and hexahedral elements with the 11 field mixed formulations, for aspect ratio 1000 and Poisson's ratio 0.45 and 0.499 , respectively.

As can be observed from the tables, the $p=2$ elements exhibit severe shear locking in this case. Comparing the results of thick and thin beams, it is evident that this phenomenon occurs solely due to the high aspect ratio (slenderness of the beam) [63, 64]. Interestingly, although not as dramatic, the MWF implementation also exhibits shear locking. On the other hand, hexahedral 


\begin{tabular}{|c|c|c|c|c|c|c|c|c|c|c|}
\hline Quantity & Refinement & $p=2$ & $p=3$ & $p=4$ & $p=5$ & $q=2$ & $q=3$ & $q=4$ & $q=5$ & 11Mixed \\
\hline \multirow{3}{*}{$u_{x}^{A}(m)$} & Coarse & 0.1603 & 0.1614 & 0.1606 & 0.1576 & 0.1621 & 0.1607 & 0.1613 & 0.1603 & 0.1682 \\
\hline & Medium & 0.1609 & 0.1606 & 0.1609 & 0.1603 & 0.1622 & 0.1607 & 0.1614 & 0.1603 & 0.1647 \\
\hline & Fine & 0.1611 & 0.1612 & 0.1611 & 0.1599 & 0.1622 & 0.1607 & 0.1614 & 0.1603 & 0.1641 \\
\hline \multirow{3}{*}{$u_{z}^{A}(m)$} & Coarse & 1.0215 & 1.0273 & 1.0274 & 0.949 & 1.0265 & 1.031 & 1.0299 & 1.0198 & 1.109 \\
\hline & Medium & 1.0256 & 1.028 & 1.0213 & 1.0175 & 1.0282 & 1.0306 & 1.0317 & 1.0197 & 1.0737 \\
\hline & Fine & 1.0256 & 1.0304 & 1.0326 & 1.0175 & 1.0286 & 1.0306 & 1.0317 & 1.0198 & 1.0659 \\
\hline \multirow{3}{*}{$F_{x z}^{A}$} & Coarse & -0.0189 & -0.0201 & -0.019 & -0.019 & -0.0203 & -0.0187 & -0.0201 & -0.0191 & -0.021 \\
\hline & Medium & -0.0195 & -0.0188 & -0.0189 & -0.0193 & -0.0203 & -0.0187 & -0.0202 & -0.0191 & -0.0205 \\
\hline & Fine & -0.0197 & -0.0198 & -0.0191 & -0.0212 & -0.0203 & -0.0187 & -0.0202 & -0.0191 & -0.0205 \\
\hline \multirow{3}{*}{$H_{z x}^{A}$} & Coarse & -0.02 & -0.02 & -0.0193 & -0.0191 & -0.0204 & -0.0192 & -0.0198 & -0.0194 & -0.0209 \\
\hline & Medium & -0.0197 & -0.0195 & -0.0194 & -0.0192 & -0.0205 & -0.0191 & -0.02 & -0.0194 & -0.0204 \\
\hline & Fine & -0.0198 & -0.019 & -0.0198 & -0.0199 & -0.0205 & -0.0191 & -0.02 & -0.0194 & -0.0205 \\
\hline \multirow{3}{*}{$\sigma_{x z}^{B}(\mathrm{~Pa})$} & Coarse & 89.1516 & -23.91 & 14.7041 & 0.2679 & 7.9494 & 39.9045 & -41.9385 & 17.966 & -25.4328 \\
\hline & Medium & 10.3683 & 60.0207 & 38.6844 & -20.1666 & 6.9024 & 27.4752 & -32.0594 & 17.139 & -18.2278 \\
\hline & Fine & -3.7742 & -90.0163 & 12.6685 & -143.9537 & 6.0727 & 27.538 & -32.0799 & 17.0549 & -4.8466 \\
\hline \multirow{3}{*}{$\sigma_{z z}^{B}(\mathrm{~Pa})$} & Coarse & 234.1627 & 260.194 & 146.468 & 244.717 & 143.7517 & 299.7924 & 199.8753 & 270.1902 & 376.1902 \\
\hline & Medium & 245.7591 & 362.475 & 203.2137 & 255.0402 & 144.1905 & 299.4855 & 200.6011 & 270.5423 & 282.167 \\
\hline & Fine & 233.4144 & 265.2387 & 177.1516 & 192.5099 & 144.4195 & 299.4976 & 200.6389 & 270.5092 & 204.6006 \\
\hline \multirow{3}{*}{$p_{h y d}^{B}(\mathrm{~Pa})$} & Coarse & 273.3164 & 279.5418 & 241.9163 & 274.1948 & 273.0657 & 273.9475 & 273.383 & 273.9117 & 402.374 \\
\hline & Medium & 273.4977 & 272.6952 & 253.7051 & 274.2576 & 273.0683 & 273.934 & 273.4753 & 273.9096 & 294.6149 \\
\hline & Fine & 273.7066 & 274.0771 & 259.1145 & 270.249 & 273.0699 & 273.9341 & 273.4752 & 273.9103 & 246.635 \\
\hline
\end{tabular}

Table 13: Comparison of high order displacement potential and 11 field mixed formulations for different kinematic and kinetic measures, for aspect ratio 10 and Poisson's ratio 0.499

elements have a consistent performance across all polynomial degrees. It is important to mention that, this phenomenon can not be attributed to the nearly incompressible nature of the material, due to two reasons. First, for $p=2$ elements, the thick beams do not exhibit locking neither at $\nu=0.45$ nor at $\nu=0.499$. Second, MWF performs specifically well for nearly incompressible scenarios and explicitly solves for the $J$ variable through an augmented Hu-Washizu variational principle [1]. Also notice that, for this aspect ratio, even $p=3$ elements exhibit some amount of locking. The oscillations in the stress on the other hand, for DPF formulations is huge, at high polynomial degrees. This is in part due to the fact that, higher order elements are much more stretched. For instance, the coarsest $p=5$ elements have an aspect ratio of 215 and the coarsest $q=5$ elements have an aspect ratio of 450 .

\begin{tabular}{|c|c|c|c|c|c|c|c|c|c|c|}
\hline Quantity & Refinement & $p=2$ & $p=3$ & $p=4$ & $p=5$ & $q=2$ & $q=3$ & $q=4$ & $q=5$ & 11Mixed \\
\hline \multirow{3}{*}{$u_{x}^{A}(m)$} & Coarse & -0.0057 & -0.037 & -0.04437 & -0.0478 & -0.0566 & -0.056 & -0.0536 & -0.0527 & 0.0005 \\
\hline & Medium & 0.0021 & -0.049 & -0.0473 & -0.0487 & -0.057 & -0.0571 & -0.0529 & -0.0532 & -0.0156 \\
\hline & Fine & 0.0017 & -0.0483 & -0.0494 & -0.0499 & -0.0572 & -0.0572 & -0.0537 & -0.0561 & -0.0252 \\
\hline \multirow{3}{*}{$u_{z}^{A}(m)$} & Coarse & 0.0437 & 2.3622 & 2.8948 & 2.9245 & 3.0755 & 3.0593 & 2.9972 & 3.0944 & 0.5598 \\
\hline & Medium & 0.0677 & 2.7467 & 2.9379 & 2.9545 & 3.0875 & 3.0916 & 2.9803 & 3.0929 & 1.672 \\
\hline & Fine & 0.2951 & 2.7054 & 2.9584 & 2.9941 & 3.0927 & 3.0956 & 3.0022 & 3.0972 & 2.094 \\
\hline \multirow{3}{*}{$F_{x z}^{A}$} & Coarse & -0.0074 & -0.0467 & -0.0435 & -0.0235 & -0.0604 & -0.0599 & -0.0589 & -0.054 & -0.0004 \\
\hline & Medium & -0.0013 & -0.0541 & -0.0437 & -0.0444 & -0.0606 & -0.0606 & -0.0586 & -0.055 & -0.0001 \\
\hline & Fine & -0.0061 & -0.0547 & -0.0537 & -0.0455 & -0.0606 & -0.0607 & -0.059 & -0.0516 & -0.0003 \\
\hline \multirow{3}{*}{$H_{z x}^{A}$} & Coarse & -0.0075 & -0.0467 & -0.04535 & -0.0235 & -0.0604 & -0.06 & -0.0589 & -0.054 & -0.0406 \\
\hline & Medium & -0.0013 & -0.0541 & -0.0438 & -0.0445 & -0.0606 & -0.0606 & -0.0586 & -0.0551 & -0.0106 \\
\hline & Fine & -0.0061 & -0.0547 & -0.0537 & -0.0456 & -0.0607 & -0.0607 & -0.059 & -0.0516 & -0.0345 \\
\hline \multirow{3}{*}{$\sigma_{x z}^{B}(\mathrm{~Pa})$} & Coarse & 18.3433 & -0.0816 & -1.2205 & -0.9087 & 0.6522 & 0.5042 & -0.4751 & 0.7071 & -0.1453 \\
\hline & Medium & -0.0358 & -1.1296 & 8.3123 & 2.6078 & 0.7813 & 0.3317 & 0.2968 & 1.1267 & 0.4453 \\
\hline & Fine & -0.1437 & -0.8996 & 1.2107 & 3.3222 & 0.892 & 0.333 & 0.2834 & 1.1086 & 0.1926 \\
\hline \multirow{3}{*}{$\sigma_{z z}^{B}(\mathrm{~Pa})$} & Coarse & -32.2983 & -15.9872 & 541.2936 & 63.4422 & 0.0401 & 0.1828 & 0.8809 & -0.7323 & 1.6931 \\
\hline & Medium & -1.173 & -10.6548 & 327.1866 & 120.7646 & -0.0127 & 0.1375 & 0.1661 & 0.2904 & 4.1625 \\
\hline & Fine & -0.3662 & 2.8504 & 23.2398 & 4.5291 & -0.0542 & 0.1445 & 0.0937 & 0.308 & 14.0885 \\
\hline \multirow{3}{*}{$p_{h y d}^{B}(\mathrm{~Pa})$} & Coarse & -24.8172 & -23.6293 & 410.2216 & 42.8566 & 2.7135 & 2.8305 & 3.7971 & 2.0094 & -4.2656 \\
\hline & Medium & -8.1182 & -16.7676 & 308.1874 & 111.348 & 2.6489 & 2.8169 & 2.8075 & 2.4095 & 5.391 \\
\hline & Fine & -6.7439 & -3.6837 & 26.1795 & 10.0663 & 2.5984 & 2.8229 & 2.7651 & 2.4154 & 15.0638 \\
\hline
\end{tabular}

Table 14: Comparison of high order displacement potential and 11 field mixed formulations for different kinematic and kinetic measures, for aspect ratio 1000 and Poisson's ratio 0.45

The conclusion drawn from the experiments so far is that, both DPF and MWF are capable of capturing the behaviour of compressible and nearly incompressible dielectric elastomers fairly well. However, for thin beams, low order DPF discretisations on tetrahedra as well MWF suffer from shear locking (resolvable through the use of a finer discretisation), while high order DPF discretisations are able to cope up with thin beams and resolve the primary variables accurately 


\begin{tabular}{|c|c|c|c|c|c|c|c|c|c|c|}
\hline Quantity & Refinement & $p=2$ & $p=3$ & $p=4$ & $p=5$ & $q=2$ & $q=3$ & $q=4$ & $q=5$ & 11 Mixed \\
\hline \multirow{3}{*}{$u_{x}^{A}(m)$} & Coarse & 0.0022 & -0.0329 & -0.0563 & -0.0564 & -0.0603 & -0.0618 & -0.0607 & -0.0618 & 0.0004 \\
\hline & Medium & 0.0022 & -0.0489 & -0.0559 & -0.0574 & -0.0642 & -0.0615 & -0.0615 & -0.0605 & -0.0194 \\
\hline & Fine & 0.0022 & -0.0434 & -0.0577 & -0.0582 & -0.0614 & -0.0616 & -0.0615 & -0.6165 & -0.0312 \\
\hline \multirow{3}{*}{$u_{z}^{A}(m)$} & Coarse & 0.0062 & 2.1839 & 2.9353 & 3.0931 & 3.1536 & 3.2548 & 3.2635 & 3.2618 & 0.5988 \\
\hline & Medium & 0.0024 & 2.496 & 2.9301 & 2.9984 & 3.2623 & 3.2082 & 2.9336 & 3.2518 & 1.8414 \\
\hline & Fine & 0.0104 & 2.8819 & 2.933 & 2.9997 & 3.2017 & 3.2099 & 3.1395 & 3.2548 & 2.3124 \\
\hline \multirow{3}{*}{$F_{x z}^{A}$} & Coarse & -0.0007 & -0.0441 & -0.0505 & -0.0515 & -0.0626 & -0.0691 & -0.0603 & -0.0621 & -0.0004 \\
\hline & Medium & -0.0002 & -0.0565 & -0.0564 & -0.0526 & -0.0643 & -0.063 & -0.0592 & -0.0044 & -0.0001 \\
\hline & Fine & -0.0002 & -0.0501 & -0.0561 & -0.0582 & -0.063 & -0.063 & -0.0613 & -0.0593 & -0.0004 \\
\hline \multirow{3}{*}{$H_{z x}^{A}$} & Coarse & -0.0007 & -0.0441 & -0.0205 & -0.0515 & -0.0627 & -0.0691 & -0.0604 & -0.0621 & -0.0448 \\
\hline & Medium & -0.0002 & -0.0565 & -0.0565 & -0.0527 & -0.0643 & -0.063 & -0.0592 & -0.0044 & -0.0115 \\
\hline & Fine & -0.0002 & -0.0501 & -0.0561 & -0.0583 & -0.063 & -0.063 & -0.0613 & -0.0593 & -0.038 \\
\hline \multirow{3}{*}{$\sigma_{x z}^{B}(\mathrm{~Pa})$} & Coarse & -0.058 & -1.0561 & 0.2593 & 0.191 & 0.7204 & 0.7001 & 0.7089 & 0.7071 & -0.2034 \\
\hline & Medium & -0.0656 & -1.022 & 5.1248 & -39.3918 & 0.8225 & 0.5512 & 0.3729 & -9.6306 & 0.6378 \\
\hline & Fine & -0.1293 & -0.8051 & 10.014 & 28.8039 & 0.8635 & 0.5506 & 0.364 & -206.3348 & 0.2668 \\
\hline \multirow{3}{*}{$\sigma_{z z}^{B}(\mathrm{~Pa})$} & Coarse & 47.1482 & -395.4936 & -33.2631 & 538.5343 & -2.8369 & 3.3726 & 3.3814 & 3.3796 & 2.1594 \\
\hline & Medium & 0.6649 & 49.3672 & -414.2999 & 2113.4412 & -4.1608 & 6.1501 & 1.802 & 2217.1176 & 4.6069 \\
\hline & Fine & -0.6984 & 13.3289 & 330.097 & 2189.0055 & -5.4436 & 6.173 & 1.4294 & 3478.8777 & 14.6644 \\
\hline \multirow{3}{*}{$p_{h y d}^{B}(\mathrm{~Pa})$} & Coarse & 38.1504 & -407.0878 & -41.0376 & 528.9243 & 0.4472 & 6.5811 & 6.5899 & 6.5881 & -5.0029 \\
\hline & Medium & -7.9517 & 40.8982 & -420.7844 & 985.5186 & -0.8781 & 9.456 & 5.1897 & 739.779 & 6.6814 \\
\hline & Fine & -9.1691 & 5.7871 & 350.556 & 710.8945 & -2.1342 & 9.4748 & 4.8289 & 1165.274 & 15.8006 \\
\hline
\end{tabular}

Table 15: Comparison of high order displacement potential and 11 field mixed formulations for different kinematic and kinetic measures, for aspect ratio 1000 and Poisson's ratio 0.499

at the expense of a huge oscillation in derived variables such as stresses. To separate the issue of volumetric and shear locking further a different set of experiments are now considered using only DPF implementation by applying an extremely high electric voltage on the patches and lowering the Poisson's ratio to $\nu=0.35$. The loading scenario for different aspect ratios is listed in Table 16.

\begin{tabular}{|l|l|l|l|}
\hline Aspect Ratio & 10 & 100 & 1000 \\
\hline Electric Voltage $(V / m)$ & $2.6 e 7$ & $1 e 5$ & $4 e 2$ \\
\hline
\end{tabular}

Table 16: Applied electric voltage as Dirichlet boundary condition for three aspect ratios

Figure 17 shows the hydrostatic pressure for the patch with aspect ratio 10 (thick) solved with the medium mesh. Once again, it can be seen that even for large deformations and finite strains, the DPF implementation with tetrahedra does not suffer from any locking, although some oscillation in the pressure could be observed, across all polynomial degrees. Similarly, Figure 18 shows the evolution of the co-factor component $H_{13}$ on the coarse mesh. For this aspect ratio, which could be considered a thin beam, DPF implementation with tetrahedra once again produces accurate results.

As evident by now, the challenging problem is the extremely thin beams with aspect ratio 1000 shown in Figure 18 . Notice that both $p=2$ and $p=3$ elements exhibit severe shear locking for this aspect ratio, confirming the fact that this locking is purely due to stretching of the elements. Only $p=4$ elements (and beyond) are able to capture this deformation accurately. Interestingly, as shown in Figure 20 the $q=2$ elements also lock at this level of deformation. Certainly, the problem of shear locking could in part be remedied by using $h$-refinement. As shown in Figure 21 , even with the fine mesh the $q=2$ elements suffer from shear locking.

The examples presented here show a clear advantage for the application of high order elements in electromechanics. Modelling of dielectric elastomeric beams with high aspect ratio is indeed a challenging problem. Mixed formulations based on convex multi-variable electro-elastic strain energies have an unrivalled performance in capturing the pressure and other derived quantities of interest, but still could suffer from shear locking at extremely high aspect ratios. Note that the performance of high order elements could still be superior if the element's aspect ratio was preserved during polynomial enrichment (which was not the case for here). The analyses perfor- 


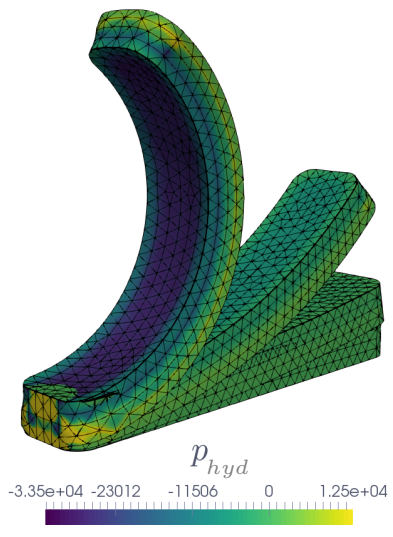

(a)

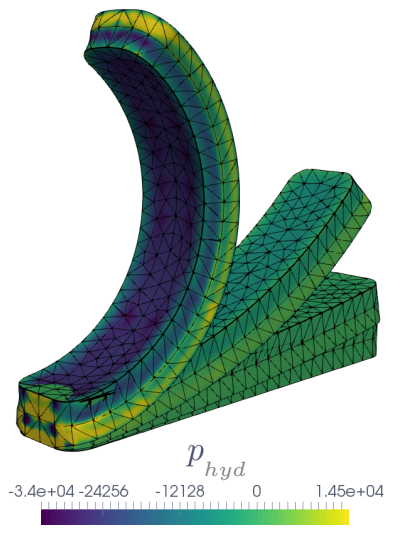

(b)

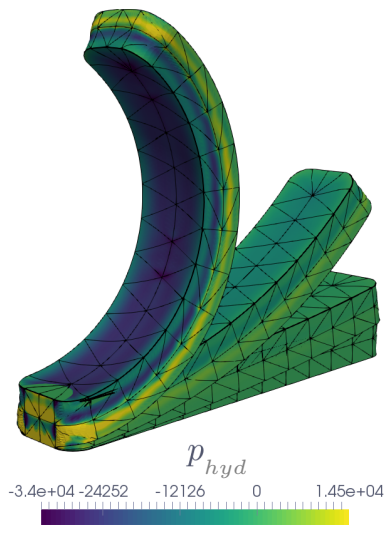

(c)

Figure 17: Voltage induced hydrostatic pressure $p_{\text {hyd }}$ for the medium mesh using a) $p=2$, b) $p=3$ and, c) $p=4$

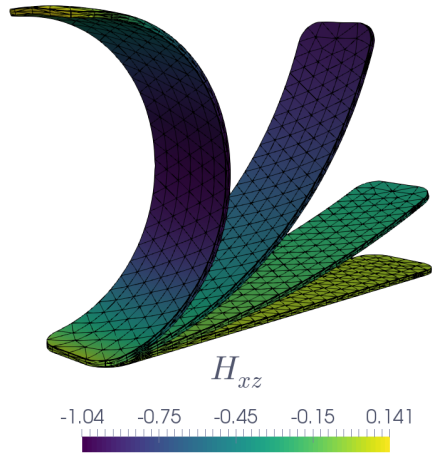

(a)

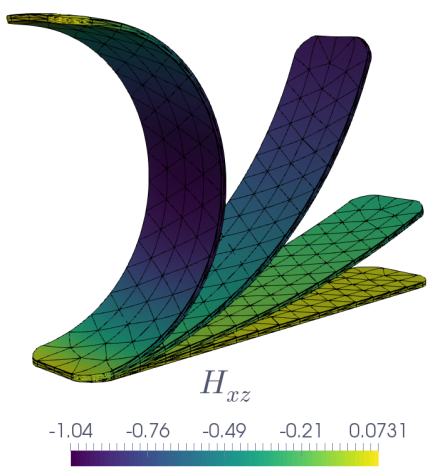

(b)

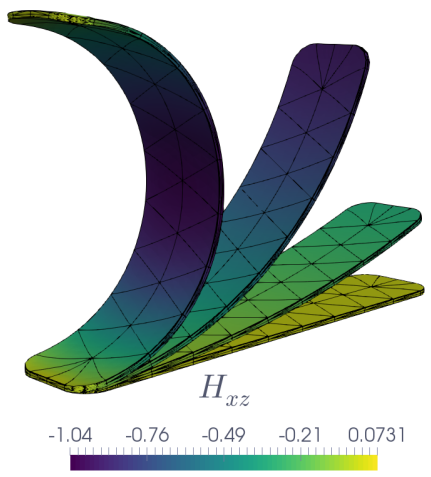

(c)

Figure 18: Voltage induced large strain $\left(H_{x z}\right)$ for the coarse mesh using a) $p=2$, b) $p=3$ and, c) $p=4$

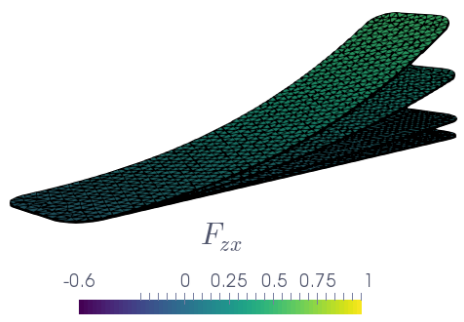

(a)

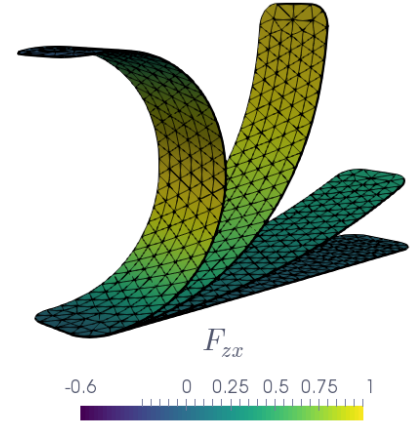

(b)

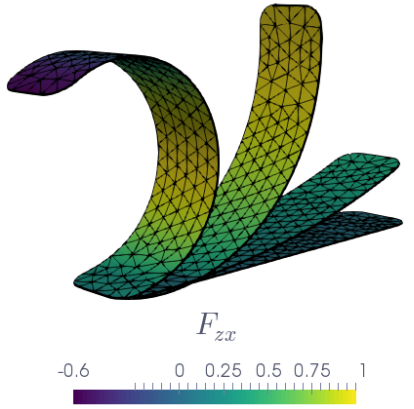

(c)

Figure 19: Voltage induced large strain $\left(F_{z x}\right)$ for the fine mesh using a) $\left.p=2, \mathrm{~b}\right) p=3$ and, c) $p=4$

med in this section was carried out with the clear goal of keeping the size of the computational domain the same across all polynomial enrichments. 


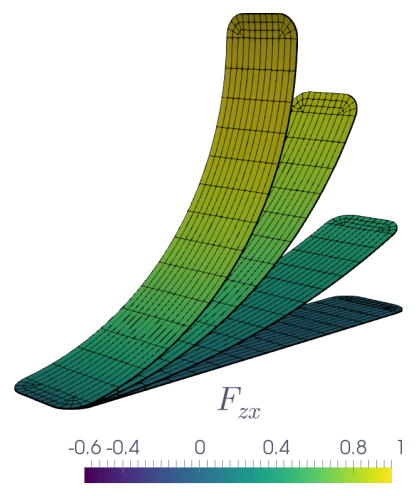

(a)

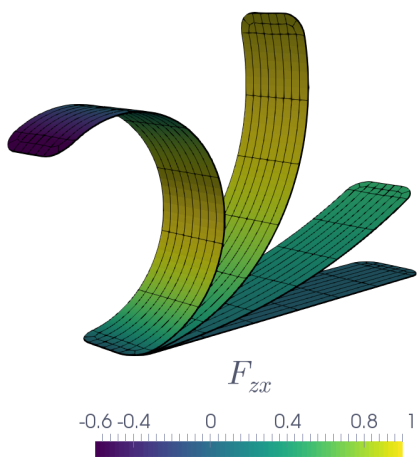

(b)

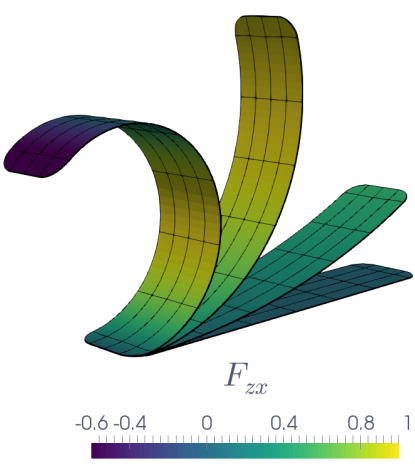

(c)

Figure 20: Voltage induced large strain $\left(F_{z x}\right)$ for the fine mesh using a) $q=2$, b) $q=3$ and, c) $q=4$

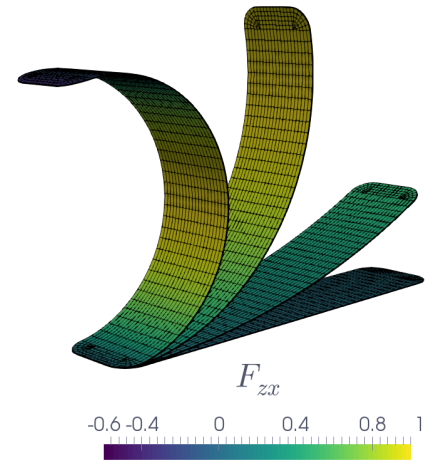

(a)

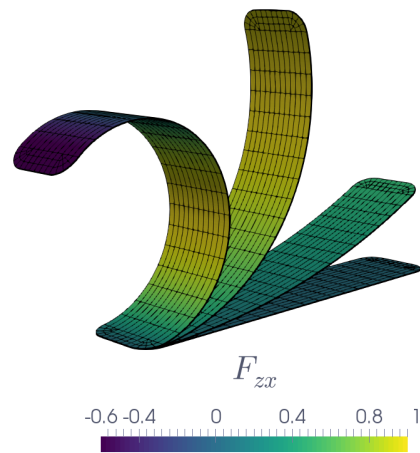

(b)

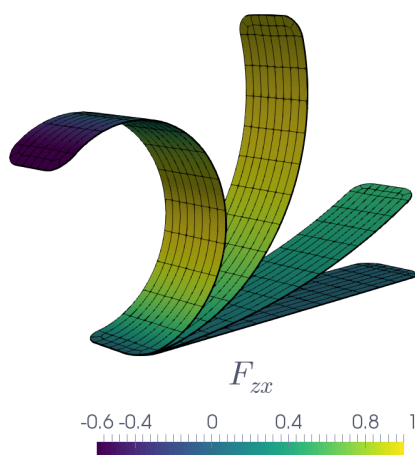

(c)

Figure 21: Voltage induced large strain $\left(F_{z x}\right)$ for the fine mesh using a) $q=2$, b) $q=3$ and, c) $q=4$

\subsection{Kernel-based data parallel code generation for electromechanics on curvilinear meshes}

Having studied the $h$ and $p$ convergence properties of the framework and its performance with respect to mixed variational principles, the objective of this section is to present some implementation details of the framework. The present high order curvilinear finite element framework is tied and closely developed on top of Fastor library [44]. Fastor (https://github.com/romeric/ Fastor) is an open source (MIT licensed) $\mathrm{C}++$ based generic tensor contraction library that can perform heavy numerical computations such as mathematical/algorithmic transformations and graph optimisations at compile time using a powerful in-built expression template engine. The transformed algorithms are then used by Fastor to generate data parallel (SIMD-vectorised) code for modern CPU and potentially GPU architectures. Recently, it has been shown in [44] that Fastor is able to generate carefully crafted data parallel code (SSE-SSE4.2, AVX-AVX2AVX512, FMA) for the local assembly of low and high order finite elements for a series of convex multi-variable electro-elastic models, exhibiting many fold performance improvement over handwritten $\mathrm{C}$ code. As described earlier, since convex multi-variable electro-elasticity models require Legendre transformation through a Newton-Raphson scheme per quadrature point to find the appropriate constitutive tensors, local assembly of the finite elements become a critical hotspot for performance optimisations (see [38, 44] for performance benchmarks).

To this effect, a problem is manufactured where the performance of the current framework (using Fastor) is studied and compared to the equivalent hand-optimised $\mathrm{C}$ code. This problem 
pertains only performance studies of numerical integration of work-conjugates (20), Hessian (22) and the subsequent quadrature point Newton-Raphson required for the Legendre transform (30) and (26), during local assembly (also see Algorithm 1). A set of similar benchmark problems has been presented in the authors' previous work on Fastor [44], where explicit code snippets for the aforementioned set of operations are presented. The interested reader is advised to consult Poya et. al. [44] and Fastor's repository, for a series of such performance studies, as for the purpose of brevity, complete implementation details of the problem are not presented here.

This performance study once again considers both curved hexahedral and tetrahedral meshes. The geometry considered for the problem is that of an electromechanical component shown in Figure 22 together with curved meshes. For the purpose of benchmark, refined meshes are chosen such that distortion qualities are all almost unity.
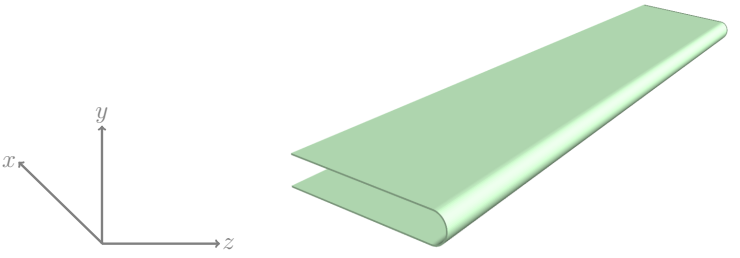

(a)

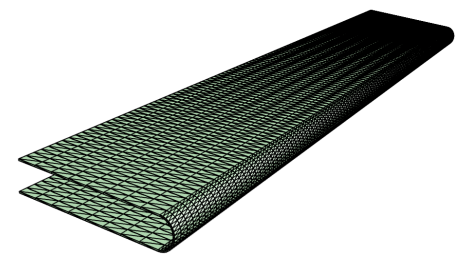

(b)

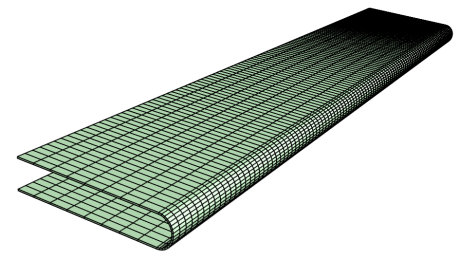

(c)

Figure 22: a) CAD geometry, b) curved tetrahedral mesh and, c) curved hexahedral mesh for electromechanical component with $1 \mathrm{~m}$ thickness, $20 \mathrm{~m}$ height (y-axis), 100m width ( $x$-axis) and $500 \mathrm{~m}$ length $(z$-axis)

Note that the tetrahedral and hexahedral meshes do not have the same number of nodes. The relevant information regarding the meshes is listed in Table 17. The geometry essentially consists of two plates connected through an extruded arc/fillet (in the $z$ direction). No boundary conditions have been applied on the arc part of the geometry. The electromechanical component is mechanically fixed at one longitudinal end and two different electric voltages are applied across the thickness of the top and bottom plates, namely $4.5 \times 10^{7} \mathrm{~V}$ for the top plate and $8.1 \times 10^{7} \mathrm{~V}$ for the bottom plate. The problem exhibits massive snap-back behaviour as the different applied voltages make the component bend initially but after the point of electromechanical instability the difference in displacements in both plates causes the component to deform in the opposite direction.

\begin{tabular}{|l|l|l|l|l|l|l|}
\hline Mesh (N. Elements) & $p / q=2$ & $p / q=3$ & $p / q=4$ & $p / q=5$ & $p / q=6$ & $p / q=7$ \\
\hline Tetrahedral (43200) & 367196 & 1223568 & 2689668 & 5082200 & 8366140 \\
\hline Hexahedral (7200) & 351276 & 1048144 & 2322900 & 4348344 & 7297276 & 12921312 \\
\hline
\end{tabular}

Table 17: Degrees of freedom associated with each polynomial degree for tetrahedral and curvilinear meshes

To study the performance of numerical integration, the stabilised convex multi-variable model (42) is chosen with material properties listed in Table 7. The problem is then analysed under the aforementioned boundary conditions and the performance of numerical integration of workconjugates $\Sigma_{\mathcal{V}}(20)$, Hessian and constitutive tensors of the internal energy $\mathcal{C}, \boldsymbol{\mathcal { Q }}, \boldsymbol{\theta}(22)$ and the quadrature point Newton-Raphson required for the Legendre transform (30) to obtain the constitutive tensors of Helmholtz-like energy $\mathcal{C}_{\Phi}, \mathcal{Q}_{\Phi}, \boldsymbol{\theta}_{\Phi}(26)$ are monitored under three different compilation flags. These correspond to the same code compiled with -03 -march=native but with auto-vectorisation turned off (i.e. -fno-tree-vectorize for gcc, -fno-vectorize for 
LLVM's clang and -no-vec for Intel's ICC), same code compiled with -03 -march=native where the compiler is permitted to auto-vectorise the code and Fastor transformed code compiled with -03 -march=native. All performance measurements have been recorded on a single core of Intel(R) Xeon(R) CPU E5-2650 v2 @2.60GHz with AVX instruction sets, 20MB private L1-cache and 32GB memory, running Ubuntu 16.04 and GCC-7, LLVM's Clang 4.0 and ICC 17.0.3 are used to compile all the codes. To keep the comparison fair, careful attention is paid to the compilers generated assembly code.

Figure 23 shows the relative speed of the code (with and without auto-vectorisation) in comparison to Fastor's generated code with all the three aforementioned compilers. Since with every polynomial enrichment the quadrature order has to be increased accordingly, as a result the computational cost of numerical integration increases almost cubically for all the cases (for threedimensional problems). Nevertheless, Fastor's data parallelism falling into the category of latency hiding techniques, hides much of this computational complexity. For both hexahedra and tetrahedra, Fastor's SIMD vectorised code shows consistently nearly $2 \mathrm{X}$ speed-up over compilers auto-vectorised code on an AVX capable processor and 3-3.5X speed up over non-vectorised (but well-optimised) code. It can also be seen from the figures, that unlike Fastor, auto-vectorisation is compiler specific and in some cases the code may not benefit from it, specifically with Intel's ICC in this case, Figure 23(e,f). On the other hand, Fastor is extremely less sensitive to the compiler (and optimisations) used. This is potentially the ultimate speed-up achievable for non-trivial codes using data parallelism on AVX architectures over non-vectorised codes (ideal speed-up is $4 \mathrm{X}$ for double precision). For complex algorithms, such as numerical integration of convex multivariable electro-elastic models, compile time code transformation and graph search optimisation are necessary to be able to achieve this performance. One again, these aspects of Fastor library are presented and benchmarked in detail in [44].

Finally, Figure 24 shows the evolution of voltage induced hydrostatic pressure $p_{\text {hyd }}$ at different loading stages, for $q=3$ hexahedral mesh. Notice the massive snap-back behaviour of system and butterfly shape it occupies at the final deformed configuration.

\subsection{Virtual prototyping of massive deformations and instabilities in dielectric elastomers through high performance numerical simulations}

The objective of this section is to numerically study the behaviour of highly stretchable dielectric elastomers undergoing massive deformations through virtual prototyping of a series of electromechanical components. One of the main goals of these set of examples is to gain insights into the onset of instabilities such as pull-in instability [24, 25] and the subsequent formation of wrinkles in dielectric elastomers. The occurrence of these phenomena have been experimentally verified by Plante and Dubowsky [24], where a large applied electric potential has led to massive deformation of a dielectric elastomer sheet with eventual partial wrinkling of the sheet. Zhao et. al. [25], Mao [26] and others [27, 28, 65] have also reported instabilities in DEs through numerical studies, albeit in relatively simplified settings. Here, an attempt is made to model dielectric elastomeric components which could potentially be applied as compliant actuators in soft robotics, medical devices and similar applications. From a computational point of view, four ingredients are needed to simulate such massive deformations in DEs namely, a) a convex multi-variable expansion of the electromechanical internal energy ensuring ellipticity $[1,2,3,16]$ b) accurate representation of the dielectric elastomeric components, c) high order finite element analyses to capture extreme deformations and finally, d) high performance kernels for rapid prototyping and modelling DEs. Having established all the aforementioned necessities, a diligent 


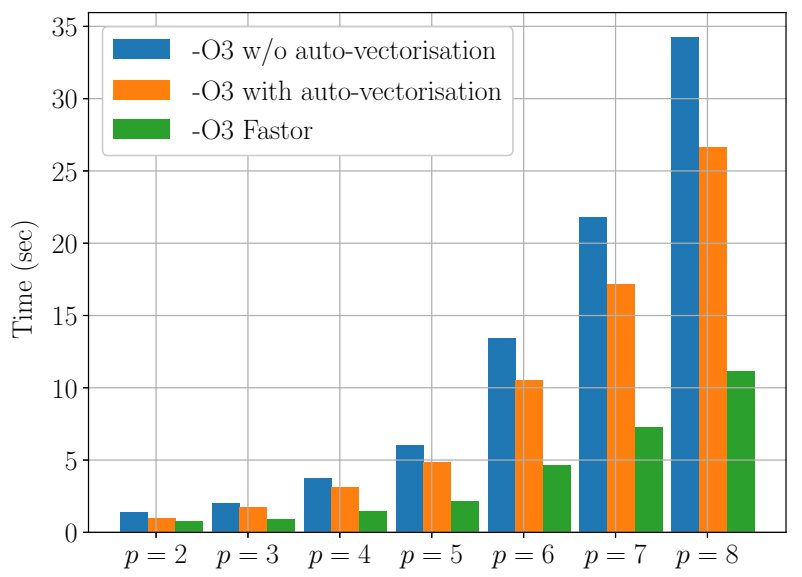

(a)

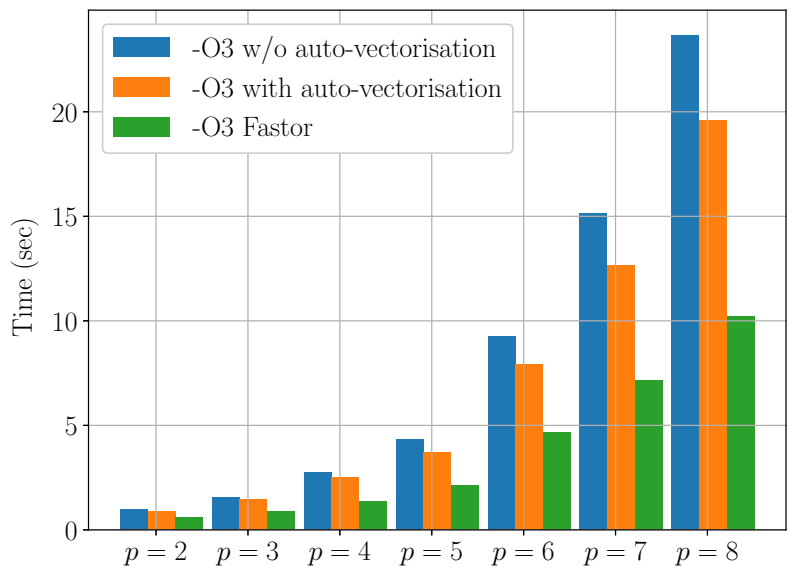

(c)

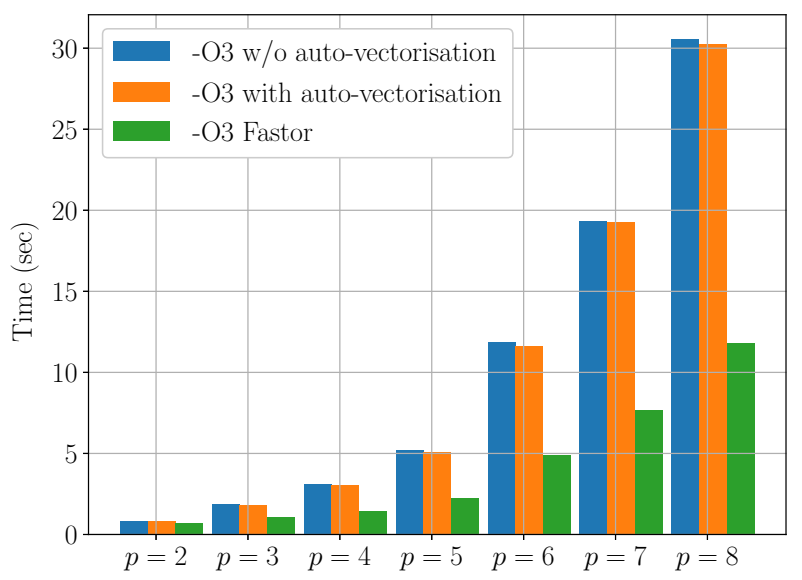

(e)

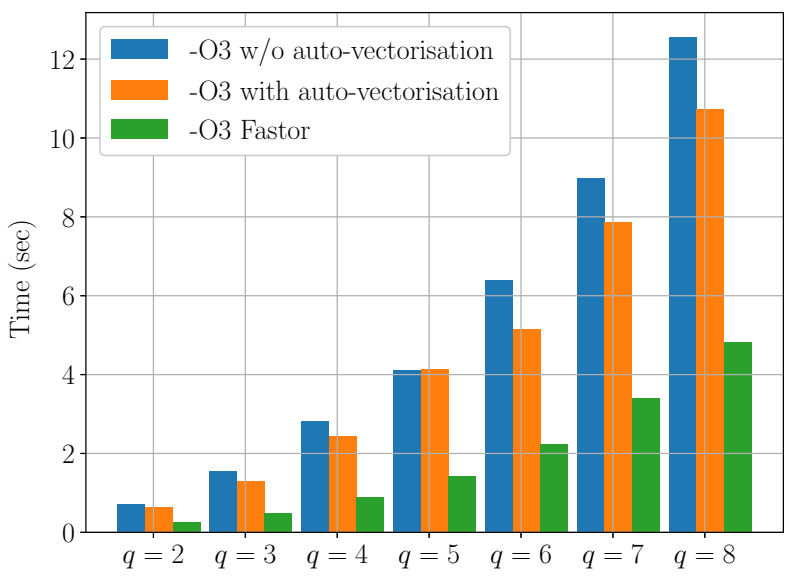

(b)

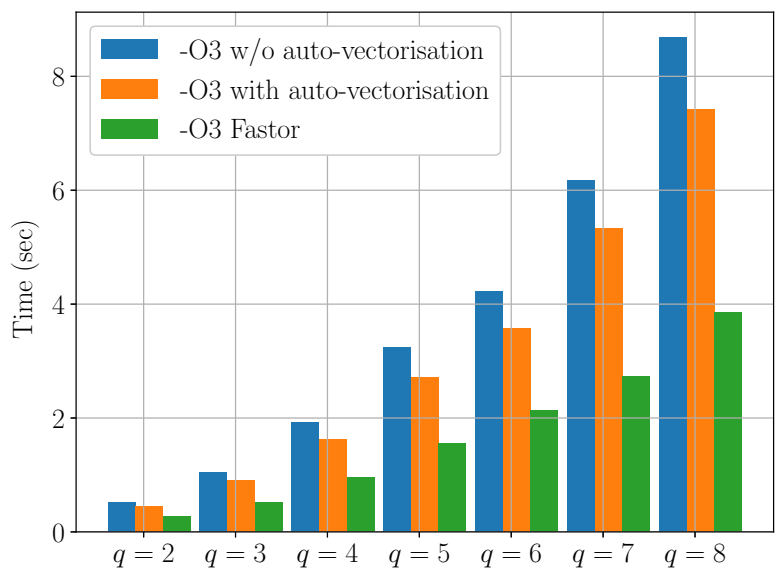

(d)

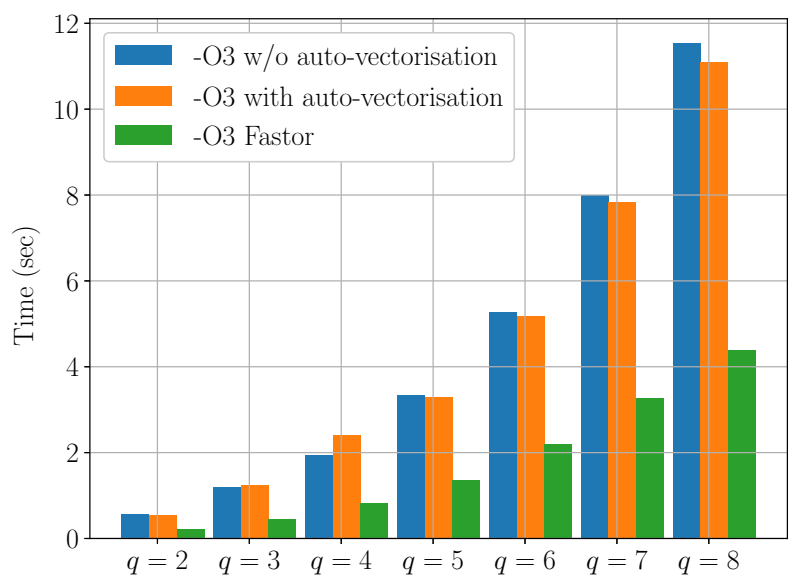

(f)

Figure 23: Speed-up achieved by compile time algorithmic transformation and data parallelism for numerical integration of work-conjugates and Hessian of convex multi-variable electro-elastic model (42) with high order curved tetrahedral and hexahedral meshes with compilers a,b) GCC, c,d) LLVM's Clang and, e,f) Intel's ICC

endeavour is put in the upcoming examples to present application-oriented numerical modelling of dielectric elastomers. Unless otherwise specified, all the examples in this section are modelled 


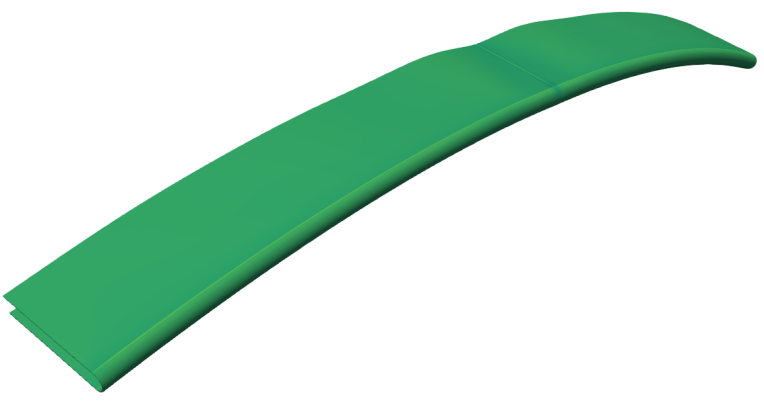

(a)

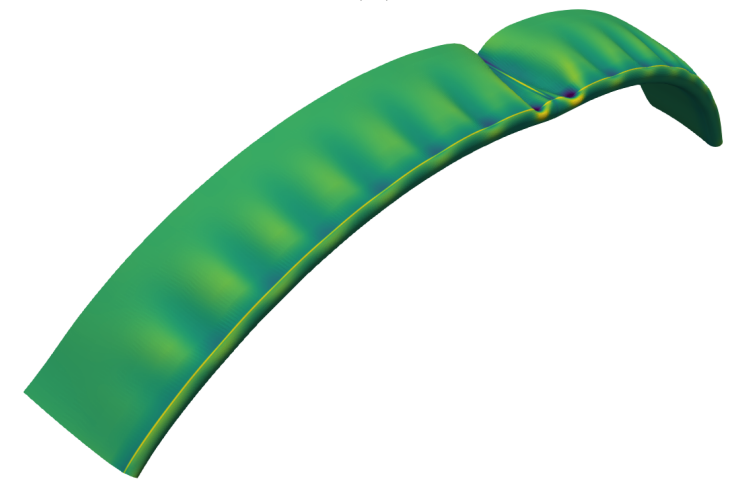

(c)

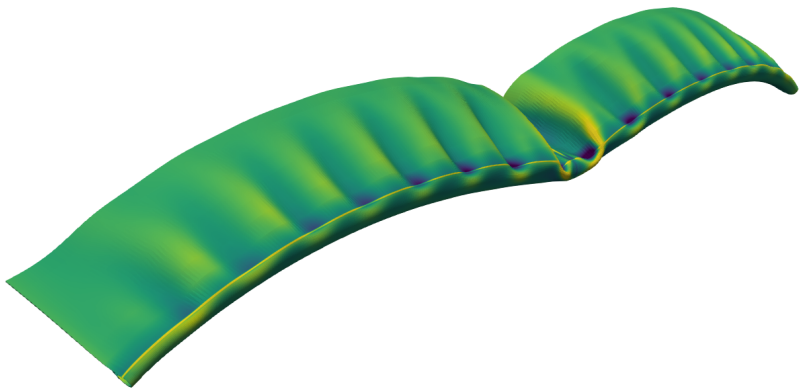

(e)

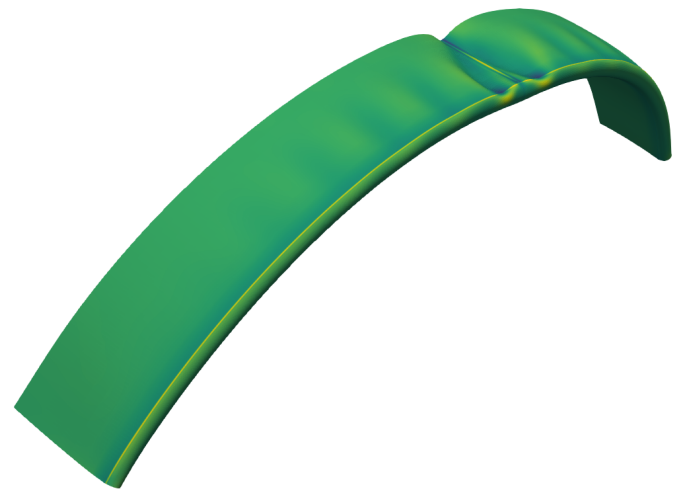

(b)

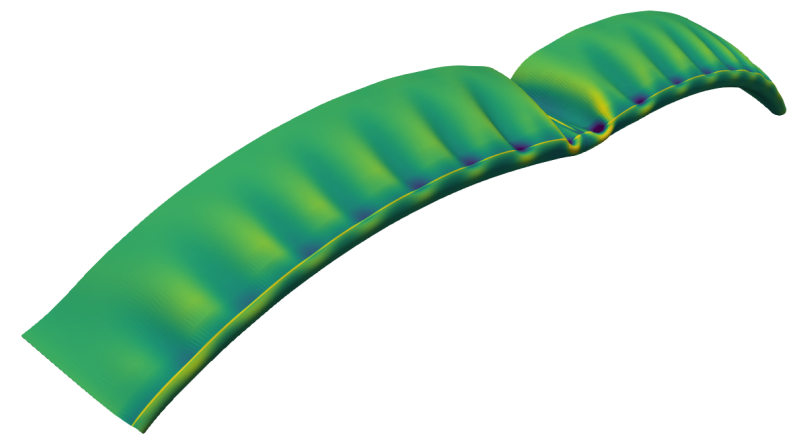

(d)

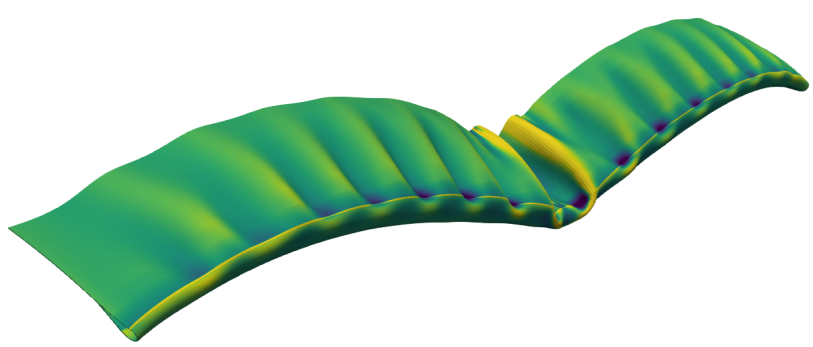

(f)

$p_{h y d}$

$\begin{array}{lllll}-6 e+04 & -37500 & -15000 & 7500 & 3 e+04\end{array}$

Figure 24: Evolution of voltage induced hydrostatic pressure $p_{\text {hyd }}$ in electromechanical component with accumulated load factor $\Lambda$ being a) $\Lambda=0.416$ b) $\Lambda=0.806$, c) $\Lambda=0.889$, d) $\Lambda=0.944$, e) $\Lambda=0.972$ and, f) $\Lambda=1.0$. Note that in the figures shown, the electromechanical component is mirrored along the $z$-axis

using the ideal dielectric elastomer material presented in (41) with material parameters listed in Table 5. For majority of these examples, the meshes are extremely fine in order to capture the formation of wrinkles (despite polynomial enrichment) and, as a consequence, the distortion quality of curvilinear meshes are almost unity. 


\subsubsection{A massively deformable dielectric cylinder}

The first example considered is that of a dielectric shell-like cylindrical structure shown in Figure $25(\mathrm{a})$ with $10 \mathrm{~m}$ outer radius, $0.333 \mathrm{~m}$ thickness and $25 \mathrm{~m}$ length, centred at $[0,0,0]^{T}$. The model is made up of $27000(p=3)$ curvilinear tetrahedral elements with 179122 points in the computational mesh corresponding to a total of 716488 degrees of freedom (shown in Figure 25(b)). An ideal dielectric elastomer is used as material model and the material properties are shown in
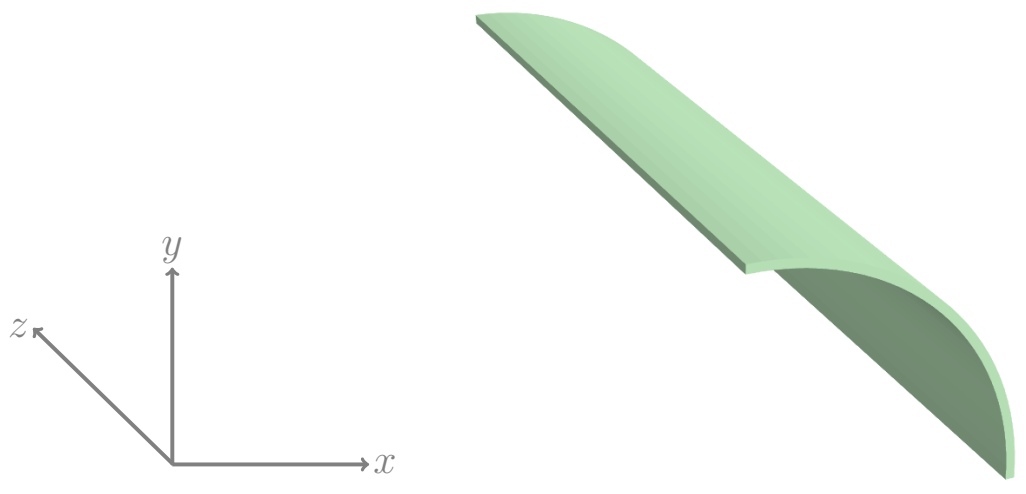

(a)

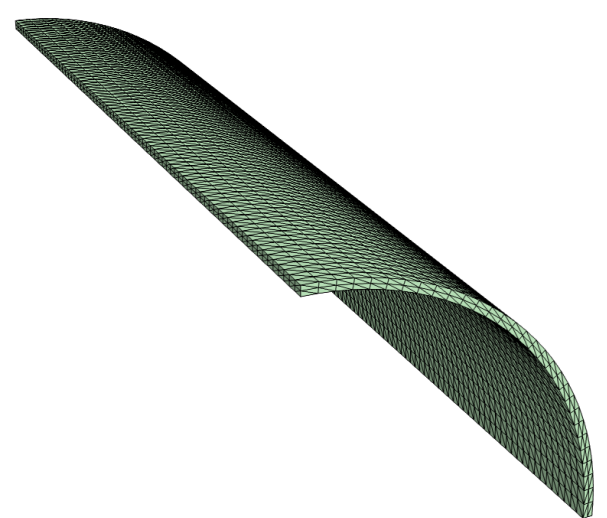

(b)

Figure 25: Geometry and a curved tetrahedral mesh of the dielectric shell-like cylindrical structure with $10 \mathrm{~m}$ radius $0.333 \mathrm{~m}$ thickness and $25 \mathrm{~m}$ length, centred at $[0,0,0]^{T}$

Table 18. The following set of boundary conditions are applied.

\begin{tabular}{|l|l|l|l|l|}
\hline$\mu_{1}(\mathrm{~Pa})$ & $\mu_{2}(\mathrm{~Pa})$ & $\lambda(\mathrm{Pa})$ & $\varepsilon_{1}^{-1}\left(\mathrm{~V}^{2} / \mathrm{N}\right)$ & $\varepsilon_{2}\left(\mathrm{~N} / \mathrm{V}^{2}\right)$ \\
\hline $1 e 5$ & 0 & $4 e 5$ & 0 & $4 \varepsilon_{0}$ \\
\hline
\end{tabular}

Table 18: Material parameters with the vacuum permittivity $\varepsilon_{0}=8.85418781 \times 10^{-12}\left(\mathrm{~N} / \mathrm{V}^{2}\right)$

$$
\begin{aligned}
u_{y} & =0[\mathrm{~m}] & & \text { at } \boldsymbol{X}=[0, y, z]^{T} ; \\
u_{x} & =0[\mathrm{~m}] & & \text { at } \boldsymbol{X}=[x, 0, z]^{T} ; \\
\boldsymbol{u} & =\mathbf{0}[\mathrm{m}] & & \text { at } \boldsymbol{X}=[10, y, z]^{T} ; \\
\varphi & =0[\mathrm{~V}] & & \text { at } R_{\text {in }} ; \\
\omega_{0} & =1.4 \times 10^{-4} \Lambda\left[\mathrm{Q} / \mathrm{m}^{2}\right] & & \text { at } R_{\text {out }},
\end{aligned}
$$

where $R_{\text {in }}=9.6667 \mathrm{~m}$ is the inner radius, $R_{\text {out }}=10 \mathrm{~m}$ is the outer radius and $\Lambda$

$$
\Lambda \in[0,1] \subset \mathbb{R}
$$

is simply a load factor controlling the application of surface charge such that, $\Lambda=0$. represents start of the load increment and $\Lambda=1$. represents end of the load increment (application of full load). The usage of load factor as a pseudo-time step to quantify the application of load is a standard practice in nonlinear quasi-static analyses [66, 67, 68]. The above non-uniform set of boundary conditions causes the cylinder to deform asymmetrically. Furthermore, the disparity 
in electric charge between the electrodes across the thickness causes the thickness to shrink. As shown in Figure 26, when the voltage is small, the elastomer deforms slightly, and the charge increases with the electric voltage approximately linearly. As the voltage increases, the elastomer's surface area expands significantly, and a small increase in the voltage adds a large amount of displacement on the cylinder. Consequently, after the voltage reaches a maximum value the voltage needed to maintain the charge drops [24]. This corresponds to the pull-in instability [25] causing regions of the cylinder to snap-back to fully new configuration possessing a different thickness. As more charge is applied at this state, the difference between the thick and thin regions causes the surface of the cylinder to fold and form wrinkles. This can be clearly observed in Figure 26(c,f). Notice that at the extreme loading state the elements closer to symmetry surfaces become considerably thinner and a complete zone of compression is formed at this region. Since

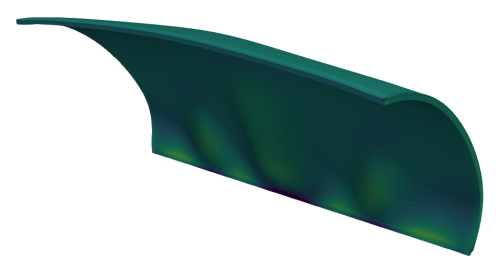

(a)

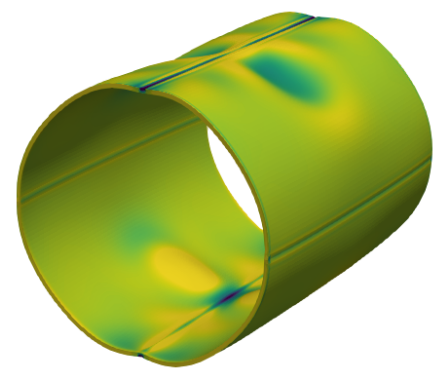

$(\mathrm{d})$
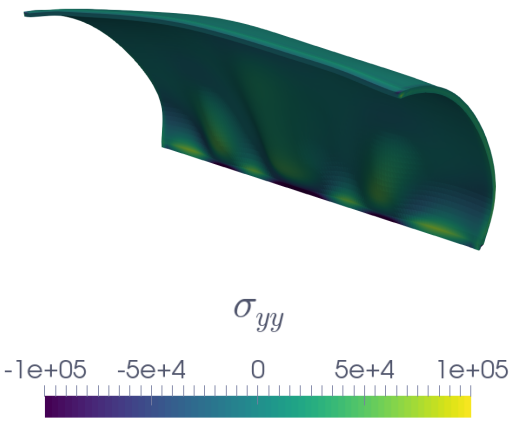

(b)

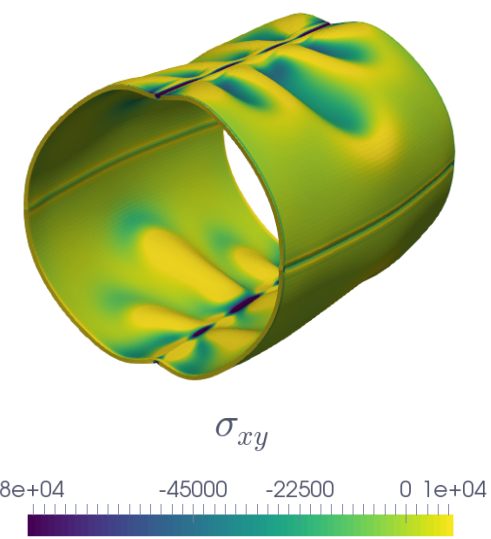

(e)

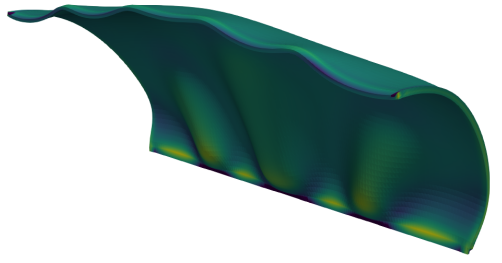

(c)

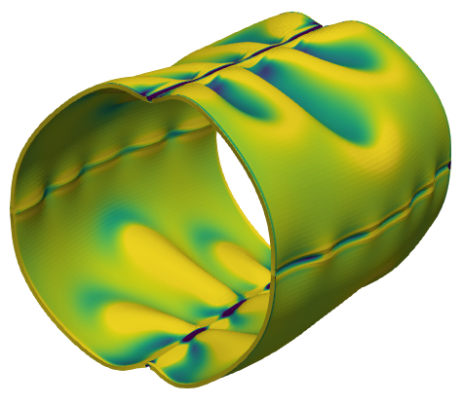

(f)

Figure 26: Evolution of charge induced stresses in dielectric cylindrical structure with accumulated load factor $\Lambda$ being a) $\sigma_{y y}$ at $\Lambda=0.678$ b) $\sigma_{y y}$ at $\Lambda=0.785$, c) $\sigma_{y y}$ at $\Lambda=1.0$ d) $\sigma_{x y}$ at $\Lambda=0.678$ e) $\sigma_{x y}$ at $\Lambda=0.785$, f) $\sigma_{x y}$ at $\Lambda=1.0$

the formation of wrinkles causes the cylinder to maintain different circumferential expansion at different regions along the length, one measure to quantify actuation property is to report the electric voltage as a function of circumferential expansion. One such graph is shown in Figure 27 for different positions along the length of the cylinder. The figure confirms the experimental findings in [24] that the formation of wrinkles is beyond the point of pull-in instability, when the surface is a mixture of thinner and thicker regions. 


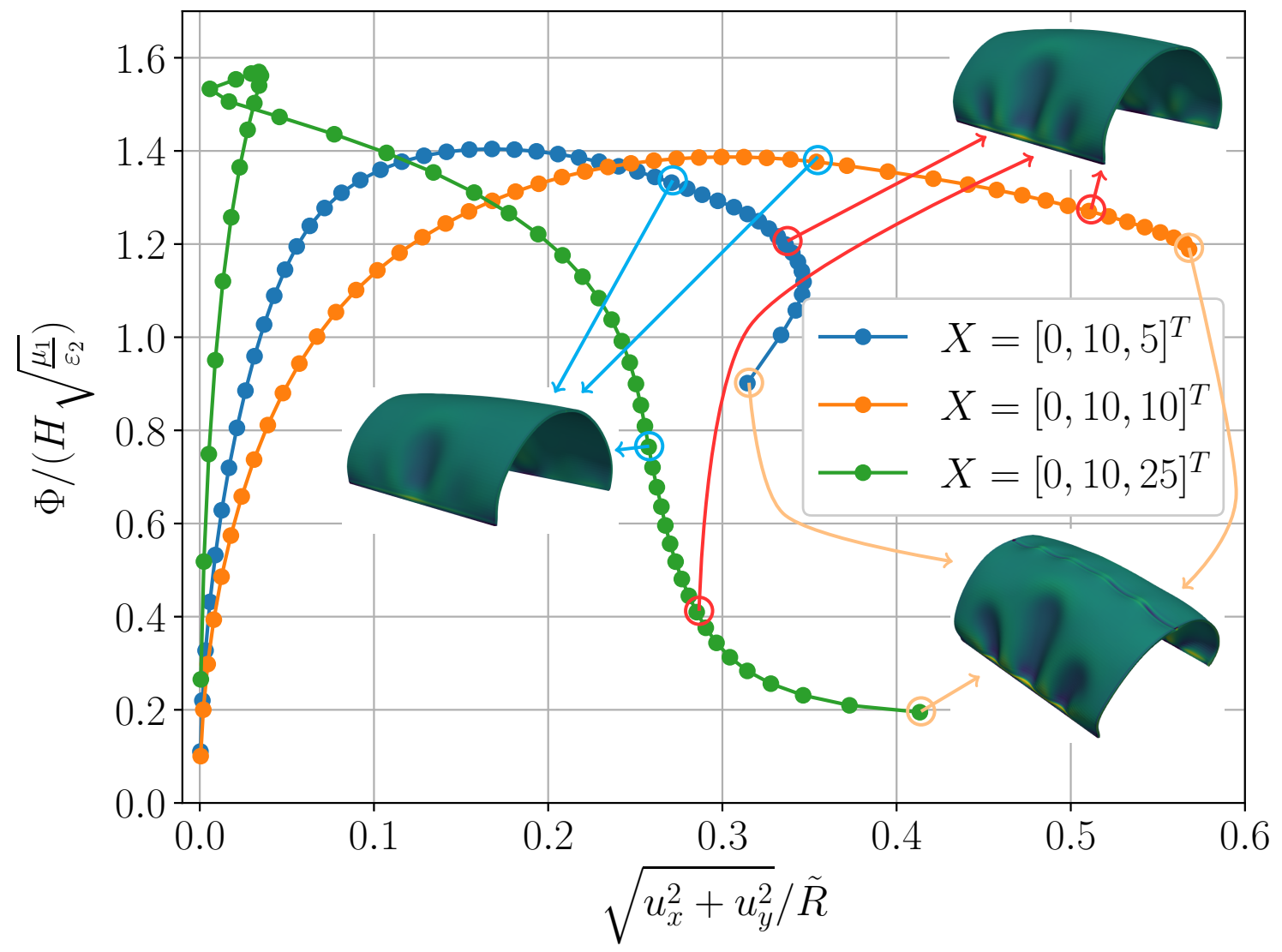

Figure 27: Non-dimensional quantity $\Phi /\left(H \sqrt{\frac{\mu_{1}}{\varepsilon_{2}}}\right)$ quantifying electric voltage as a function of circumferential expansion, showing points of snap-through, pull-in instability and formation of coarse wrinkles in dielectric elastomer. $H$ stands for thickness and $\tilde{R}=9.8333$ is the averaged radius accounting for thickness stretch

Next, two $q=3$ hexahedral meshes are chosen for the cylinder and two sets of boundary conditions are applied on each one respectively. The meshes correspond to $1.5 \mathrm{M}$ and $3.2 \mathrm{M}$ degrees of freedom, approximately. The first set of boundary conditions is similar to the previous analysis, however instead of surface charge now an electric potential of $5.2 \times 10^{7} \mathrm{~V}$ is applied at the inner radius. The second set of boundary conditions corresponds to

$$
\begin{array}{ll}
\boldsymbol{u}=\mathbf{0}[\mathrm{m}] & \text { at } \boldsymbol{X}=[0, y, z]^{T} ; \\
\boldsymbol{u}=\mathbf{0}[\mathrm{m}] & \text { at } \boldsymbol{X}=[x, 0, z]^{T} ; \\
\varphi=0[\mathrm{~V}] & \text { at } R_{\text {in }} ; \\
\varphi=5.2 \times 10^{7} \Lambda[\mathrm{V}] & \text { at } R_{\text {out }},
\end{array}
$$

where $\Lambda$ is now the load factor quantifying the application of electric potential for the nonlinear quasi-static analysis. Due to symmetry, only $1 / 8$ th of geometries are analysed. Figure 28 shows the evolution of the $F_{x z}$ component of the deformation gradient tensor with voltage history. The first observation is that since the boundary condition is now through applied voltage and not electric charge as in the previous analysis, the voltage will always constantly increase and 
hence there is no negative or downward slope in voltage vs strain curves, up until the point of electric breakdown. The second observation from Figure 28 is that, unlike in the case of applied charge where the surfaces near symmetry boundary conditions become thinner, here these surfaces undergo twisting to accommodate for the constant applied electric voltage. The formation of coarse wrinkles at $[0, x, y]^{T}$ is not present in this case.

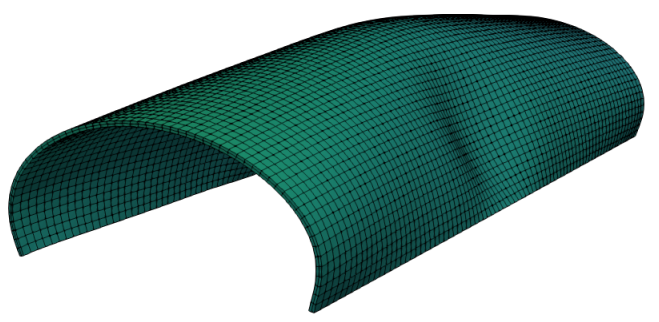

(a)

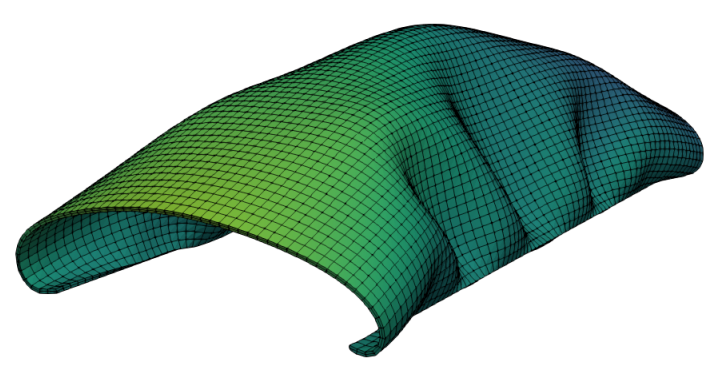

(c)

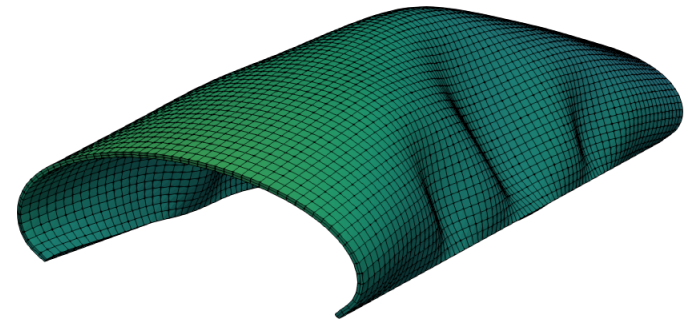

(b)

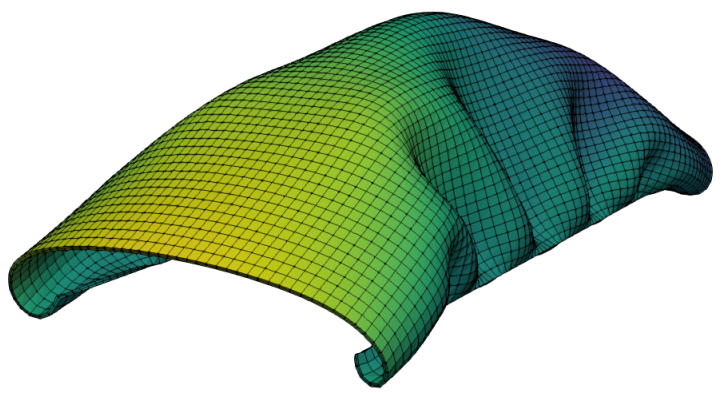

(d)

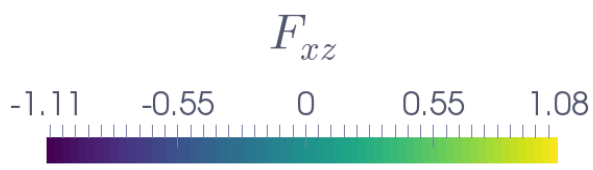

Figure 28: Evolution of voltage induced strain $F_{x z}$ in dielectric cylindrical structure with accumulated load factor $\Lambda$ being a) $\Lambda=0.538$ b) $\Lambda=0.769$, c) $\Lambda=0.923$ and, d) $\Lambda=1.0$

Finally, unlike the two previous analyses, the third example with the second set of boundary conditions for the hexahedral mesh mentioned above is completely uniform in terms of loading. As can be seen in Figure 29 this set of boundary conditions imposes a homogeneous circumferential expansion. However, once the electric voltage is high enough, coarse wave-like wrinkles starting from the central point along the length of the cylinder are formed propagating towards the two ends of the cylinder.

The above three examples of cylindrical shell-like DEs pinpoint the different actuation properties that can be activated different sets of boundary conditions. In addition, they verify the capability of the current framework to cope with extremely fine meshes and high polynomial enrichment to capture massive deformations and wrinkling in dielectric elastomers.

\subsubsection{Capsule}

The next example considered is that of a dielectric capsule, shown in Figure 30(a,b) centered at $[0,0,0]^{T}$ with an in-plane radius of $10 \mathrm{~m}$, thickness of $0.5 \mathrm{~m}$ and a bulging factor of $2 \mathrm{~m}$ in the out of plane direction. The mesh considered (shown in Figure 30(c)) has 10112 elements and for an interpolation degree $q=3$ there are 365584 points in the computational mesh which corresponds 


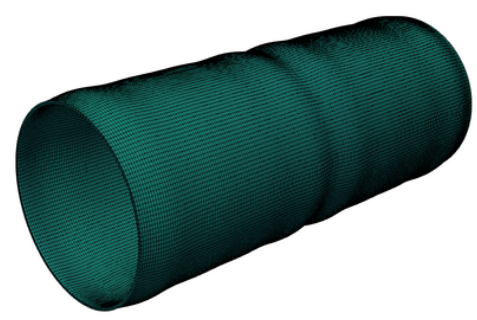

(a)

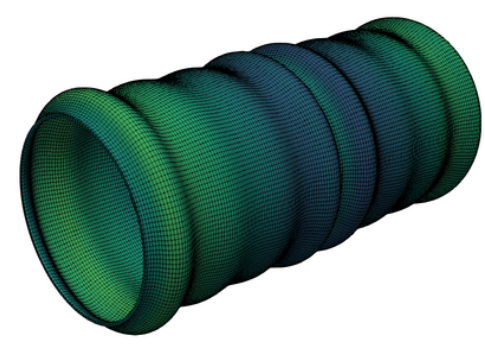

(d)

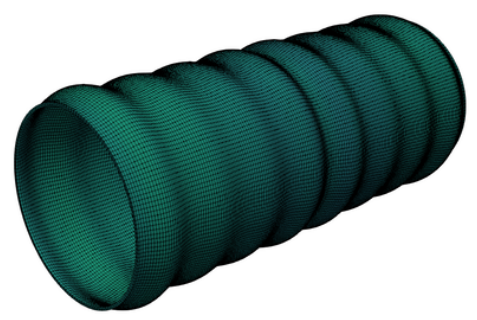

(b)

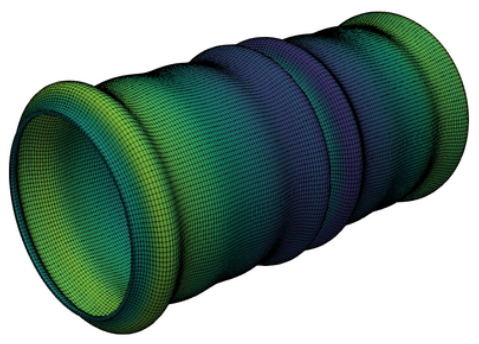

(e)

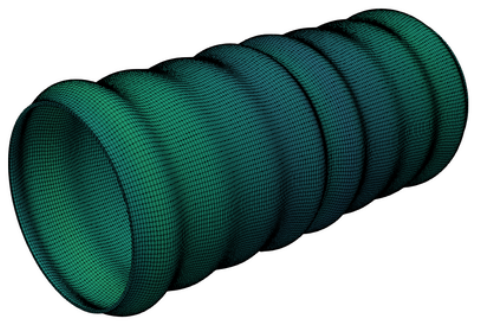

(c)

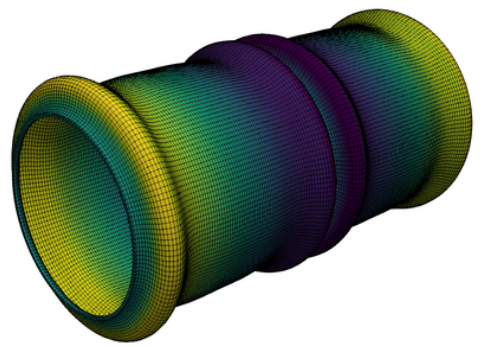

(f)

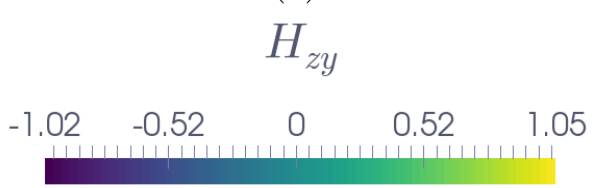

Figure 29: Evolution of voltage induced strain (co-factor of the deformation gradient) $H_{z y}$ in dielectric cylindrical structure with accumulated load factor $\Lambda$ being a) $\Lambda=0.490$ b) $\Lambda=0.686$, c) $\Lambda=0.784$, d) $\Lambda=0.882$, e) $\Lambda=0.941$ and, f) $\Lambda=1.0$

to 1462336 degrees of freedom. Due to symmetry, only $1 / 4$ th of the geometry is analysed. A set of symmetric mechanical Dirichlet boundary conditions are applied on the mesh with an applied electric voltage across the thickness, as described in the following.

$$
\begin{aligned}
\boldsymbol{u} & =\mathbf{0}[\mathrm{m}] & & \text { at } \boldsymbol{X}=[0, y, z]^{T} ; \\
u_{z} & =0[\mathrm{~m}] & & \text { at } \boldsymbol{X}=[x, 0, z]^{T} \\
\varphi & =0[\mathrm{~V}] & & \text { at } R_{\text {in }} \\
\varphi & =2.5 \times 10^{7} \Lambda[\mathrm{V}] & & \text { at } R_{\text {out }} .
\end{aligned}
$$

This problem is in particular interesting in shape and pattern forming through actuation. Figure 31 shows the hydrostatic pressure at different loading stages. As can be observed the capsule undergoes massive deformation to adopt a squared shape from an initial circular configuration, as the electric voltage is applied. The capsule deforms significantly when the electric voltage is high to adopt the new shape. After the point of snap-through as more voltage is applied, a compression zone is formed at the centre. As the electric voltage is increased, this compression zone rapidly moves from the centre towards the edges of the deformed capsule signalling the propagation of compressed band through a highly inflated capsule. The occurrence of this phenomenon is indeed analogous to the ones reported in the previous section, in that, after the point of pull-in instability the thicker regions expand at the expense of the thinner region resulting in zones of compression and propagation of pressure as a wave through the capsule. 


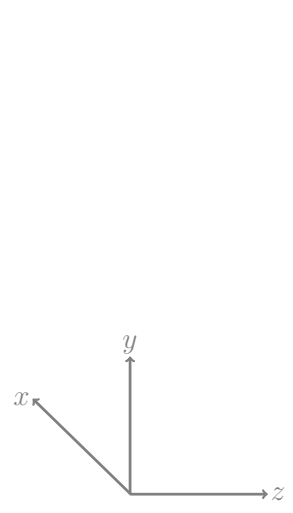

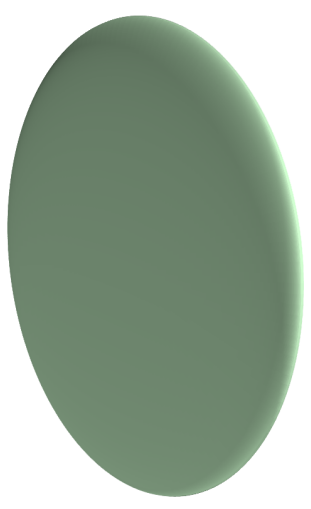

(a)

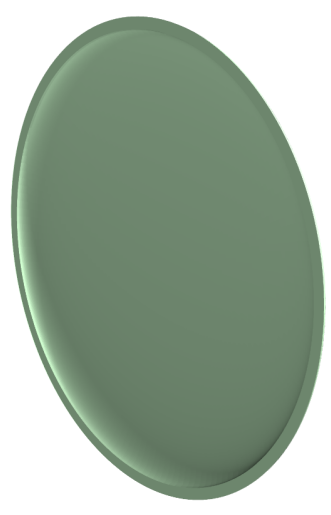

(b)

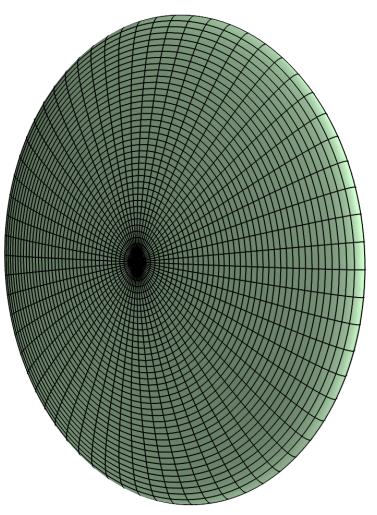

(c)

Figure 30: Dielectric capsule centred at $[0,0,0]^{T}$ with an in-plane radius of $10 \mathrm{~m}$, thickness of $0.5 \mathrm{~m}$ and a bulging factor of $2 \mathrm{~m}$ a,b) CAD geometry and c) $q=3$ structured hexahedral mesh

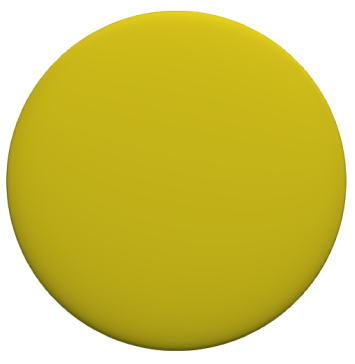

(a)

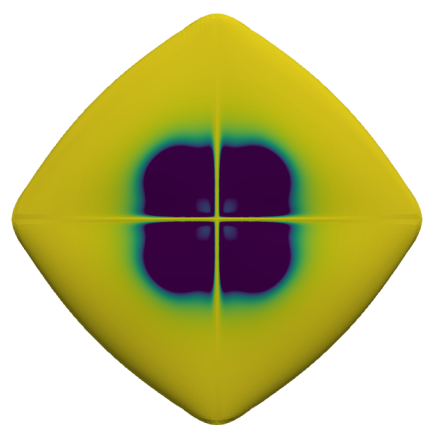

(e)

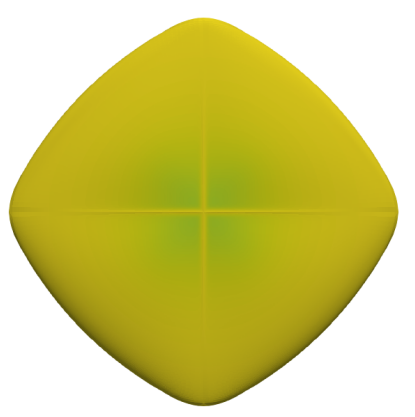

(b)

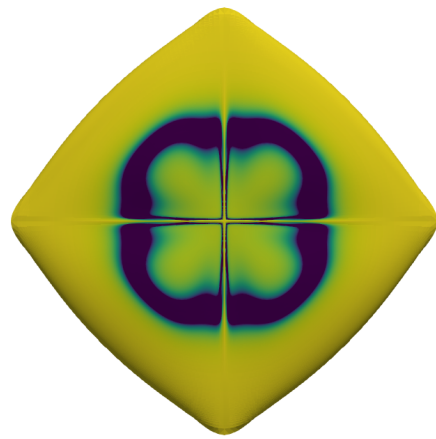

(d)

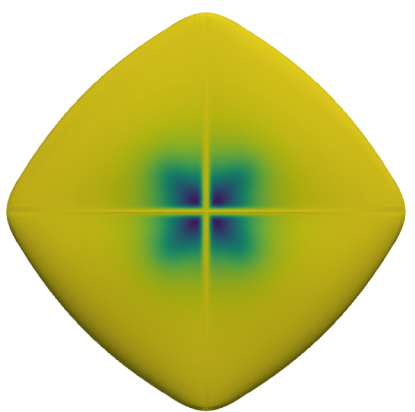

(c)

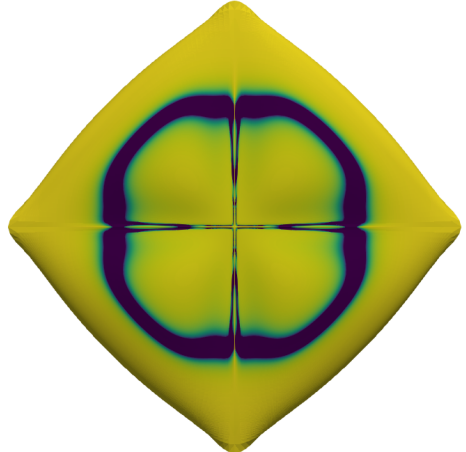

(f)

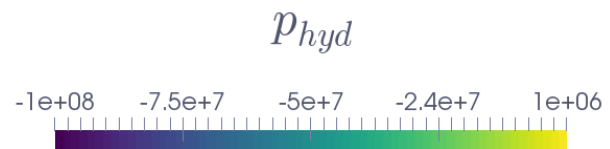

Figure 31: Evolution of voltage induced hydrostatic pressure in dielectric capsule with the accumulated load factor $\Lambda$ being a) $\Lambda=0.411$, b) $\Lambda=0.767$, c) $\Lambda=0.823$, d) $\Lambda=0.882$, e) $\Lambda=0.946$ and, f) $\Lambda=1.0$

\subsubsection{Capturing folds and coarse wrinkles in dielectric plates with and without inclusions}

The objective of this study is to show the effect of inclusions and the capability of the current high order curvilinear framework in capturing folds and coarse wrinkles with very coarse meshes 
through polynomial enrichments. To this effect, two dielectric plates are considered one without holes or inclusions and one with multiple circular inclusions distributed non-homogeneously through the plate, as shown in Figure 32. The mesh considered for the plate without inclusion is an extremely coarse $12 \times 12 \times 1$ structured hexahedral mesh (with only one element across the thickness). For a polynomial degree of $q=6$ there are 37303 points in the computational mesh corresponding to a total of 149212 degrees of freedom. For the plate with inclusions, the mesh considered is an unstructured tetrahedral with 2147 elements, as shown in Figure 32(d). For an interpolation degree of $p=4$, there are 26943 points in the computational mesh corresponding to a total of 107772 degrees of freedom.

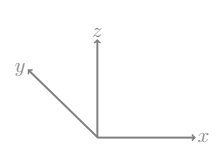

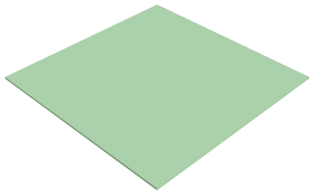

(a)

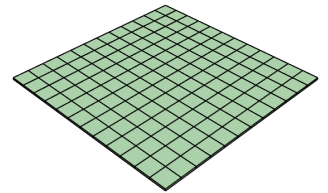

(b)

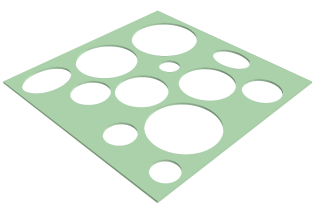

(c)

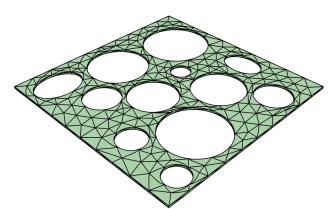

(d)

Figure 32: Geometries and meshes for the dielectric plates of size $100 \times 100 \times 1 \mathrm{~m}^{3}$, a,b) without inclusions and, c,d) with inclusions

The following set of boundary conditions are applied on both plates

$$
\begin{array}{ll}
\boldsymbol{u}=\mathbf{0}[\mathrm{m}] & \text { at } \boldsymbol{X}=[0, y, 0]^{T} ; \\
\boldsymbol{u}=\mathbf{0}[\mathrm{m}] & \text { at } \boldsymbol{X}=[100, y, 0]^{T} ; \\
\boldsymbol{u}=\mathbf{0}[\mathrm{m}] & \text { at } \boldsymbol{X}=[x, 0,0]^{T} ; \\
\boldsymbol{u}=\mathbf{0}[\mathrm{m}] & \text { at } \boldsymbol{X}=[x, 100,0]^{T} ; \\
\varphi=0[\mathrm{~V}] & \text { at } \boldsymbol{X}=[x, y, 0]^{T} ; \\
\varphi=4.98 \times 10^{7} \Lambda[\mathrm{V}] & \text { at } \boldsymbol{X}=[x, y, 1]^{T} .
\end{array}
$$

Figure 33 shows the evolution of deformation in the plate (without inclusions) at multiple loading stages and Figure 34 shows the final configuration of the plate. The formation of folds and coarse wrinkles can be clearly seen from the figures. Notice how the high order curvilinear elements are able to capture this intrinsic property of dielectric elastomers with a very coarse discretisation.

Similarly, Figure 35 shows the evolution of strain component $F_{x z}$ in the plate with inclusions at multiple loading stages and Figure 36 shows the final configuration of the plate. The formation of folds can be clearly seen from the figures. However, unlike in the case of plate with no inclusions, the deformation pattern is completely non-uniform and the plate does not deform as much. Once again, notice how the high order curvilinear elements are able to capture foldings in dielectric elastomers with an increased level of detail despite a coarse discretisation.

While folding and the formation of single layer coarse wrinkles can be captured accurately using the high order curvilinear elements, as presented in the next section, multi-layer wrinkling and the propagation and nucleation of extremely fine wrinkles would still require a refined computational mesh.

\subsubsection{Dielectric thin film undergoing massive wrinkling}

The objective of this final example is to study the voltage induced instability in a thin dielectric elastomeric film undergoing massive formation, propagation and nucleation of wrinkles. 


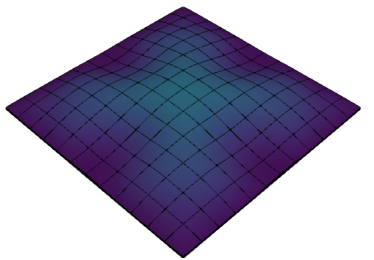

(a)

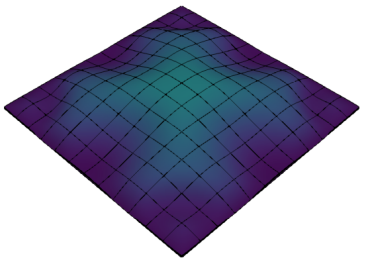

(b)

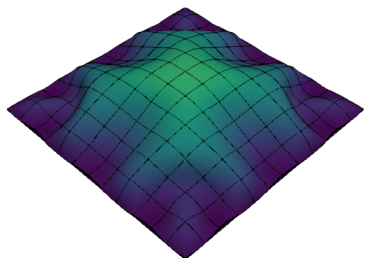

(c)

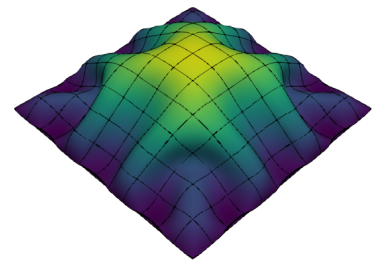

(d)

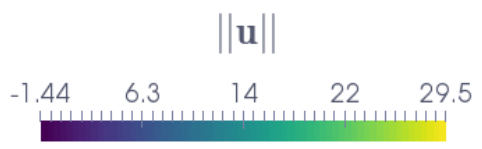

Figure 33: Evolution of norm of displacements in dielectric plate with the accumulated load factor $\Lambda$ being a) $\Lambda=0.482$, b) $\Lambda=0.602$, c) $\Lambda=0.843$ and, d) $\Lambda=1.0$
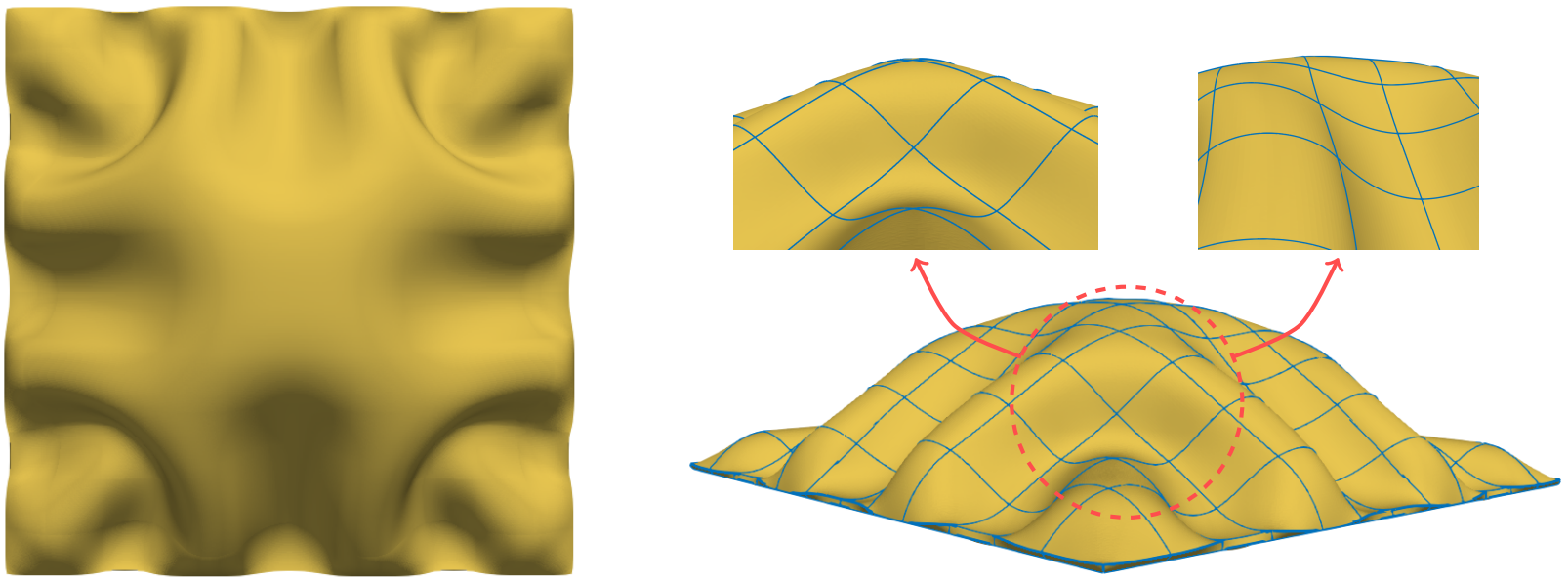

Figure 34: Formation of folds in dielectric plate (at the final deformed configuration) captured on a $12 \times 12 \times 1$ hexahedral mesh with $q=6$ polynomial interpolation

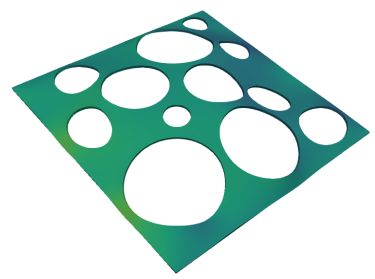

(a)

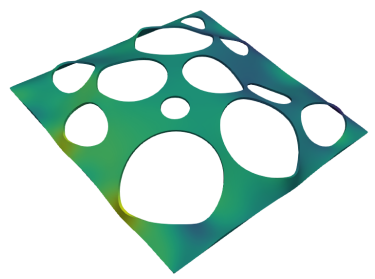

(b)

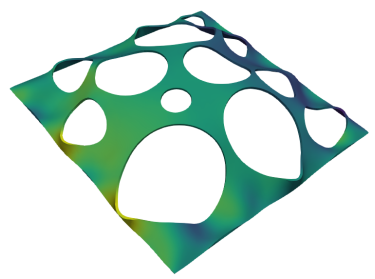

(c)

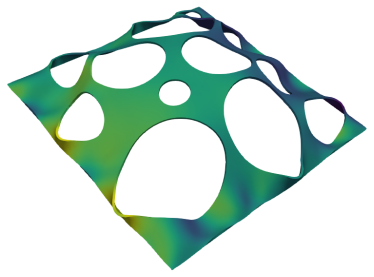

(d)

$$
\begin{aligned}
& F_{x z} \\
& \begin{array}{ccccc}
-1.07 & -0.52 & 0.029 & 0.58 & 1.13 \\
\hline
\end{array}
\end{aligned}
$$

Figure 35: Evolution of strains $F_{x z}$ in dielectric plate with inclusions with the accumulated load factor $\Lambda$ being a) $\Lambda=0.482$, b) $\Lambda=0.602$, c) $\Lambda=0.843$ and, d) $\Lambda=1.0$ 

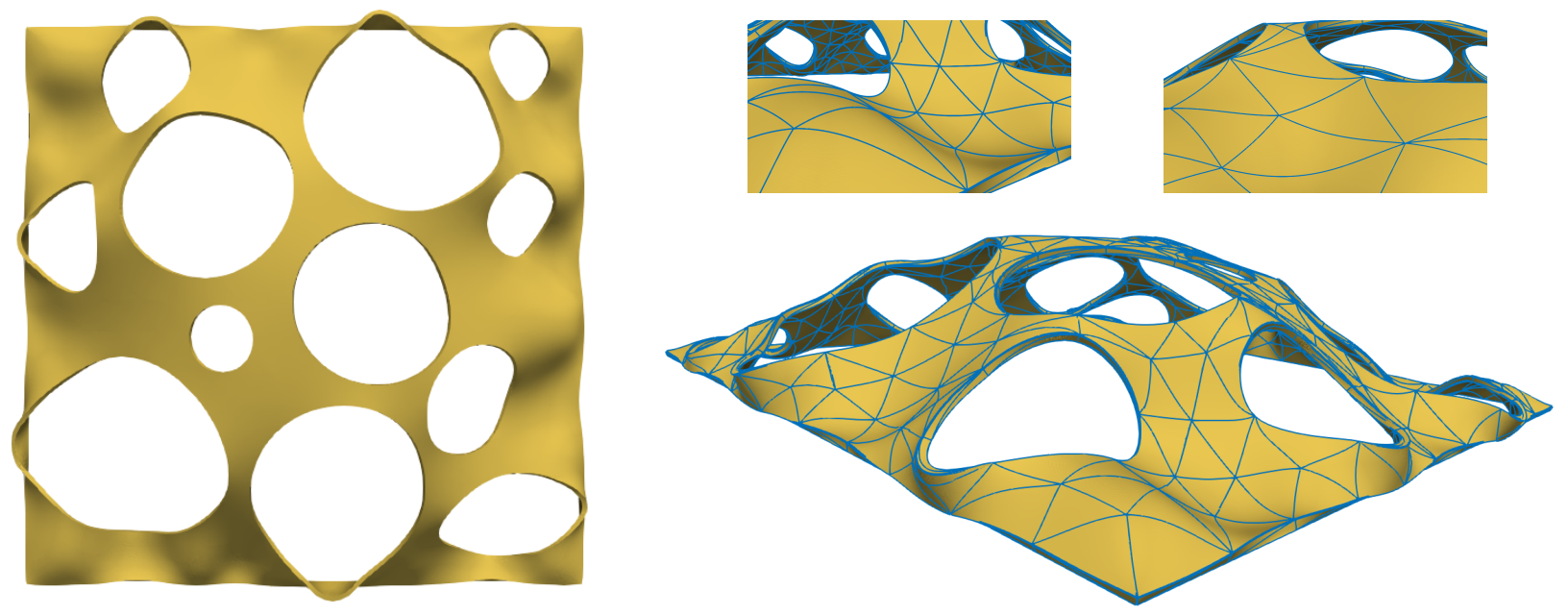

Figure 36: Formation of folds in dielectric plate with inclusions (at the final deformed configuration) captured on an unstructured tetrahedral mesh with $p=4$ polynomial interpolation

Instabilities in the form of wrinkling have also been studied in [26, 27, 28]. For the analysis, a hexahedral mesh with two polynomial enrichments is considered namely $q=2$ and $q=6$ with 268140 and 1.4M degrees of freedom, respectively. The latter allows for a highly detailed propagation of wrinkles through the film in terms of geometry and solution accuracy. The CAD geometry and mesh of the film are shown in Figure 37 with $100 \mathrm{~m}$ radius and $1 \mathrm{~m}$ thickness centred at $[0,0,0]^{T}$.
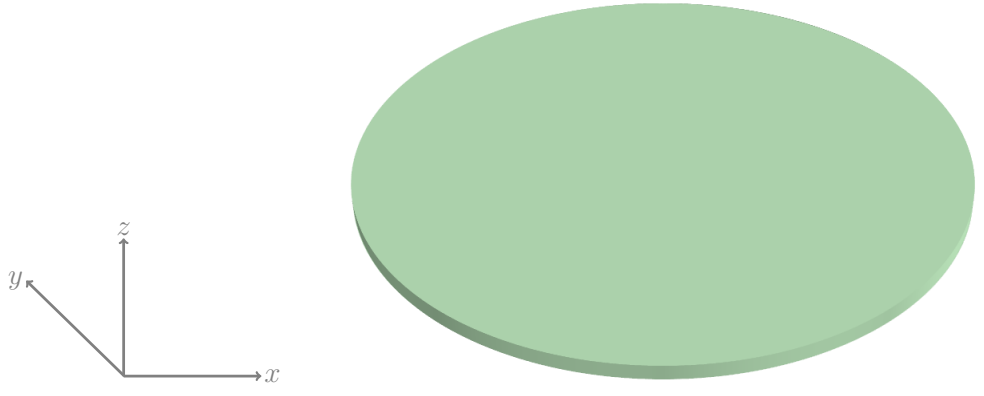

(a)

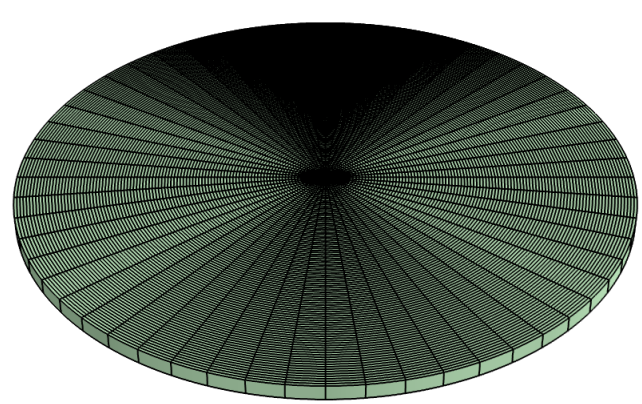

(b)

Figure 37: Dielectric thin film with $100 \mathrm{~m}$ radius and $1 \mathrm{~m}$ thickness centred at $[0,0,0]^{T}$, a) CAD geometry and, b) $q=2$ mesh

The following set of boundary conditions is applied on the film

$$
\begin{aligned}
& \boldsymbol{u}=\mathbf{0}[\mathrm{m}] \\
& \text { at } \boldsymbol{X}=[x, y, 0]^{T} \text { s.t. } x^{2}+y^{2}=100^{2} \text {; } \\
& \varphi=0[\mathrm{~V}] \\
& \varphi=5 \times 10^{7} \Lambda[\mathrm{V}] \\
& \text { at } \boldsymbol{X}=[x, y, 0]^{T} \text {; } \\
& \text { at } \boldsymbol{X}=[x, y, 1]^{T} \text {. }
\end{aligned}
$$

This above set of boundary conditions essentially implies fixing the mechanical variables at the outer boundary of the base of the film and applying an electric voltage across the thickness of the film. This forces the film to bend perpendicularly and expand in area. The electric voltage 
is applied adaptively on the film through 1000 load increments. Figure 38 shows the evolution of stress components $\sigma_{x z}$ and $\sigma_{y z}$ in dielectric film for $q=2$ mesh. As can be observed under low electric voltage, the region near the boundary of the circular film starts bulging up. This allows the film to expand in area and shrink in thickness in this region. As the voltage is increased the thick regions surrounding the thin ones start to expand and bulge facilitating the formation and further propagation of wrinkles. Under further increase in voltage all the regions start to possess similar thickness and the film straightens again allowing for nucleation of many wrinkles. The process of wrinkling starts once again, when the voltage is further increased. The process keeps repeating itself as voltage keeps increasing up until the point of complete electric breakdown. Note that the formation of deep channels near the boundary require polynomial enhancement despite a very fine mesh to capture the massive voltage induced bending caused by wrinkles.

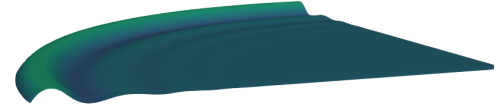

(a)

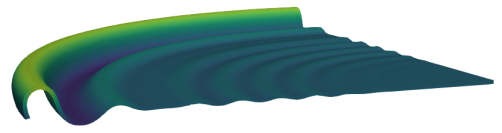

(e)

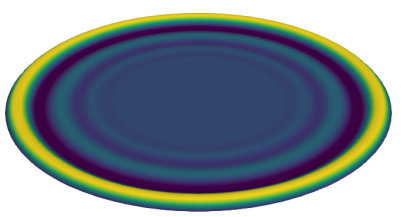

$(\mathrm{g})$

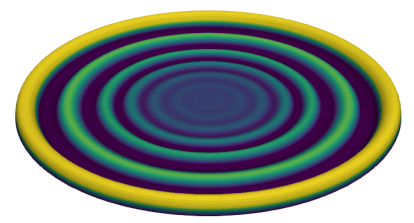

(j)

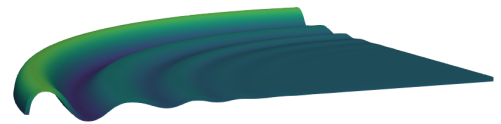

(b)

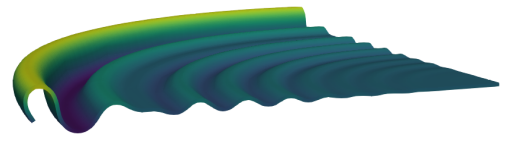

(d)

$$
\sigma_{x z}
$$
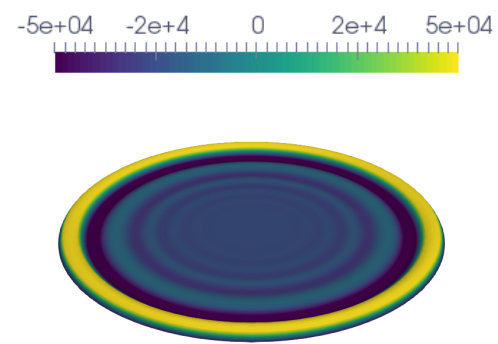

(h)

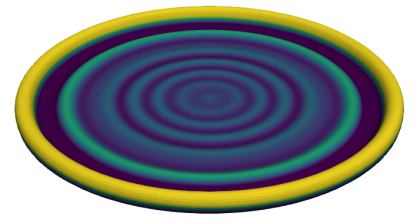

(k)

$\sigma_{y z}$

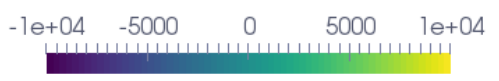

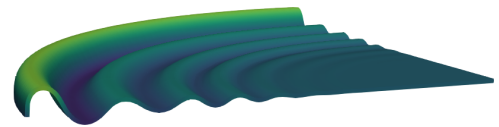

(c)

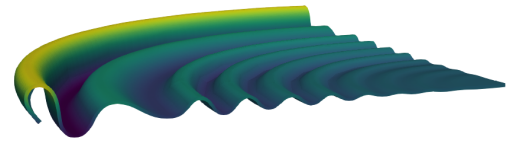

(f)

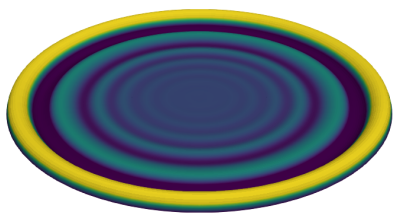

(i)

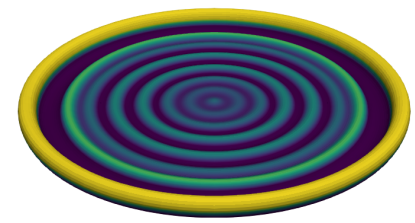

(1)

Figure 38: Evolution of voltage induced stress components $\sigma_{x z}(\mathrm{a}, \mathrm{b}, \mathrm{c}, \mathrm{d}, \mathrm{e}, \mathrm{f})$ and $\sigma_{y z}(\mathrm{~g}, \mathrm{~h}, \mathrm{i}, \mathrm{j}, \mathrm{k}, \mathrm{l})$ in dielectric film with the accumulated load factor $\Lambda$ being a,g) $\Lambda=0.580$, b,h) $\Lambda=0.725, \mathrm{c}, \mathrm{i}) \Lambda=0.798, \mathrm{~d}, \mathrm{j}) \Lambda=0.870$, e,k) $\Lambda=0.943$ and, f,l) $\Lambda=1.0$

The same problem is then analysed using $q=6$ elements, however now after the formation a certain number of wrinkles in the film, the load is released. Figure 39 shows the deformation 
history of the film together with the evolution of hydrostatic pressure. As the load is released, the deep channels around the boundary start to move inwards, leaving a thick bended layer behind. As the whole load cycle is completed the film starts flattening and forming a plate with multiple tiny wrinkles on the surface.

Finally, the aspect ratio of the film is decreased from $1 / 100$ to $1 / 20$ i.e. the radius is decreased to $20 \mathrm{~m}$ and the same problem with release load cycle is analysed using the $q=6$ mesh. The deformation pattern is the same as in the previous analysis, however, since now there is little room for the formation of multiple thick and thin regions, wrinkling is limited. As shown in Figure 40 a deep channel is formed near the boundary that starts propagating inwards till it reaches the centre. The film starts occupying a balloon shape at this stage. As the voltage is increased, the centre of the film starts bending in the opposite direction, allowing the formation of another layer of wrinkles to propagate outwards. Once the wrinkle is propagated through the film by reaching the boundaries, another layer of wrinkles starting from the centre starts propagating outwards. The process is repeated and in the process, multiple wrinkles start nucleating before hitting the boundary. Under the completion of the release cycle, the film starts flattening leaving multiple extremely fine wrinkles behind on the surface.

Overall, the problem of analysing the formation of wrinkles in dielectric elastomers is a challenging one, in that it requires a very fine mesh. With coarse meshes it is possible to capture folds using high order curvilinear elements, however multi-layer wrinkling might not even occur using coarse meshes. Even high polynomial enrichments do not allow for a wave like deformation of a single element in a wrinkled state, and hence $h$-refinement is absolutely necessary. Where polynomial enrichment really helps is in massive bending of single elements during the formation of deep channels. The examples discussed afore, pinpoint the robustness and the high performant capability of the framework in capturing massive deformations and wrinkling in dielectric elastomers with a remarkable level of detail.

\section{Concluding remarks}

A high order finite element implementation of the convex multi-variable electro-elasticity for large deformations large electric fields simulations and its particularisation to the case of small strains through a staggered scheme is presented. Accurate geometrical representation through a high performance curvilinear finite element framework based on a posteriori mesh deformation technique is developed to accurately discretise the underlying displacement-potential variational formulation. The performance of the method under near incompressibility and bending actuation scenarios is analysed with extremely thin and highly stretched components and compared to the performance of mixed variational principles. Although convex multi-variable constitutive models are elliptic and hence, materially stable for the entire range of deformations and electric fields, other forms of physical instabilities are not precluded in these models. In particular, physical instabilities present in dielectric elastomers such as pull-in instability, snap-through and the formation, propagation and nucleation of wrinkles and folds are numerically studied with a detailed precision in this paper, verifying experimental findings. In this context, the combination of $h$ and $p$ refinement proves to be essential to capture the inherent instabilities in dielectric elastomers. While the formation of folds and coarse wrinkles can be accurately captured by high order curvilinear elements using extremely coarse meshes, nucleation of wrinkles and multi-layer wrinkling require mesh refinement in addition to polynomial enrichment. For the case of small strains, the staggered scheme is shown to capture the electrostrictive behaviour of EAPs fairly well with 


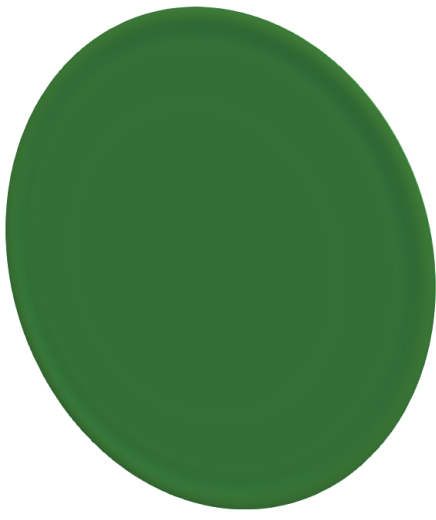

(a)

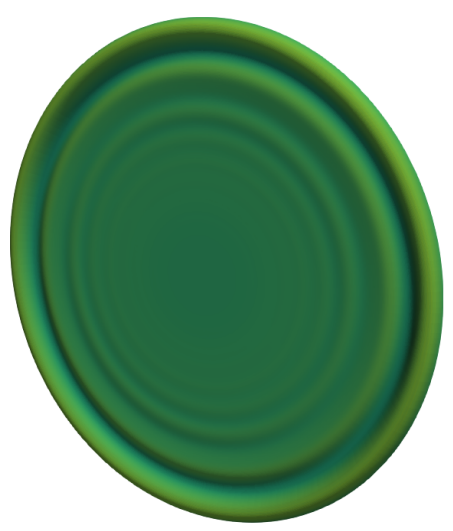

(d)

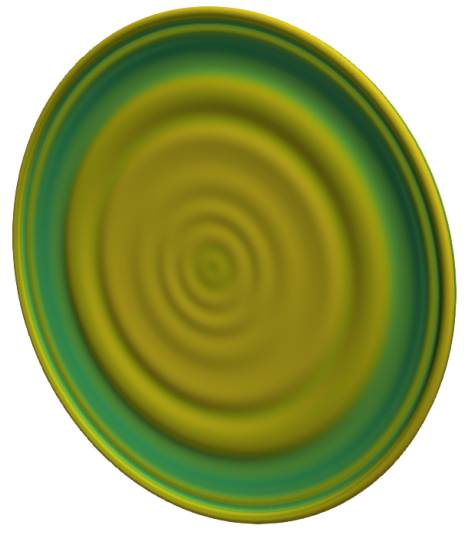

(g)

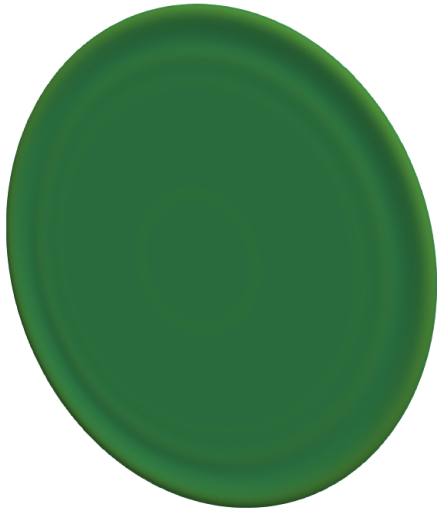

(b)

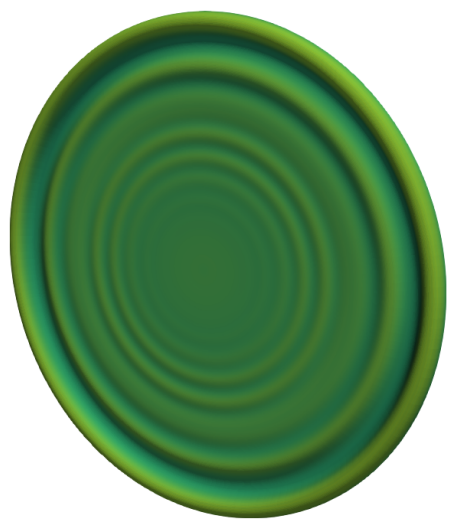

(e)

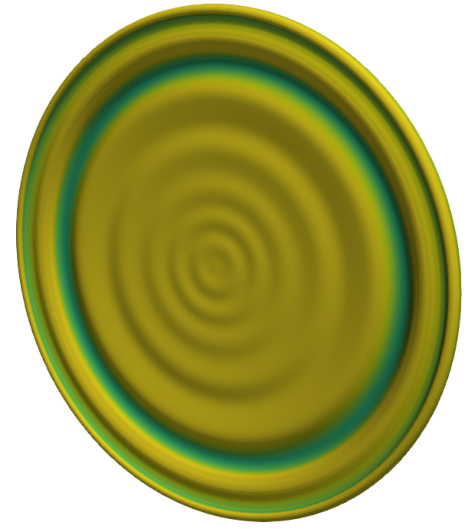

(h)

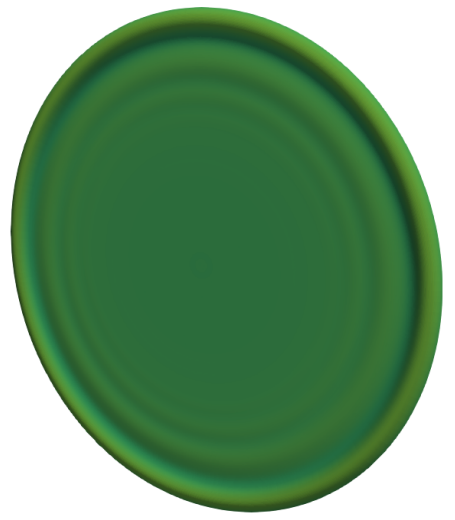

(c)

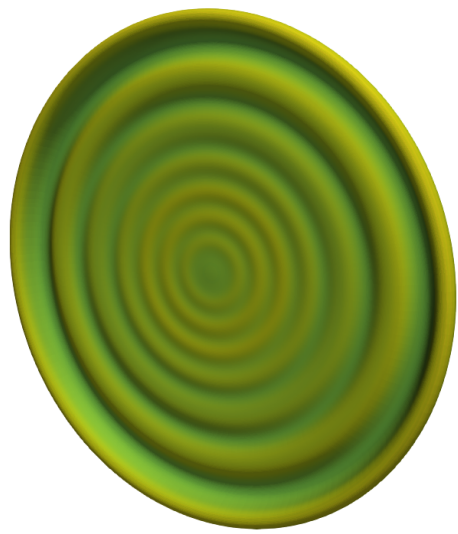

(f)

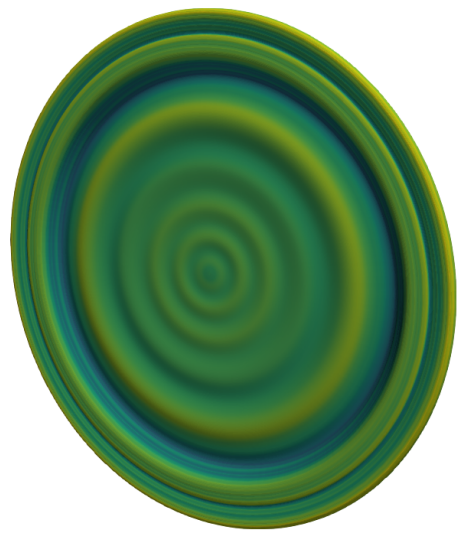

(i)

$p_{\text {hyd }}$

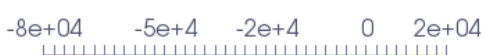

Figure 39: Evolution of voltage induced hydrostatic pressure in dielectric film with the accumulated load factor $\Lambda$ being a) $\Lambda=0.221$, b) $\Lambda=0.332$, c) $\Lambda=0.443$, d) $\Lambda=0.554$, e) $\Lambda=0.665$, f) $\Lambda=0.887$, g) $\Lambda=0.943$, h) $\Lambda=0.971$, and, i) $\Lambda=1.0$ 

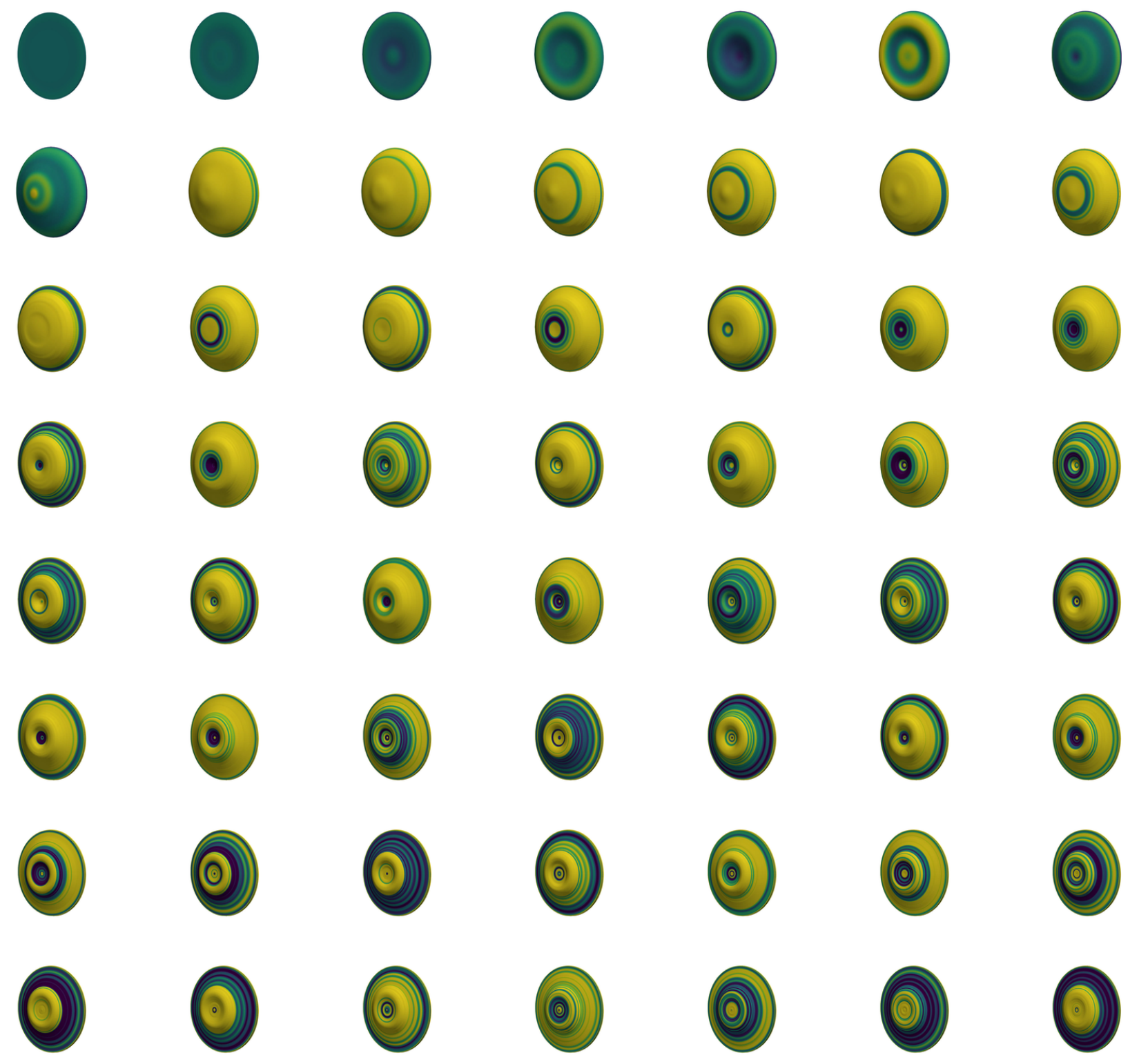

$p_{\text {hyd }}$

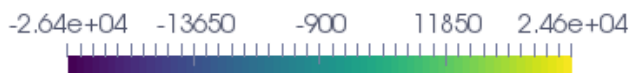

Figure 40: Evolution of voltage induced hydrostatic pressure in dielectric film with the accumulated load factor $\Lambda$ being (left to right - top to bottom) $\Lambda=0.0, \Lambda=0.124, \Lambda=0.249, \Lambda=0.374, \Lambda=0.499, \Lambda=0.624, \Lambda=0.749$, $\Lambda=0.874, \Lambda=0.886, \Lambda=0.899, \Lambda=0.911, \Lambda=0.918, \Lambda=0.921, \Lambda=0.924, \Lambda=0.928, \Lambda=0.93, \Lambda=0.934$, $\Lambda=0.936, \Lambda=0.94, \Lambda=0.942, \Lambda=0.949, \Lambda=0.952, \Lambda=0.955, \Lambda=0.958, \Lambda=0.96, \Lambda=0.962, \Lambda=0.964$, $\Lambda=0.965, \Lambda=0.966, \Lambda=0.968, \Lambda=0.969, \Lambda=0.97, \Lambda=0.971, \Lambda=0.972, \Lambda=0.974, \Lambda=0.975, \Lambda=0.976$, $\Lambda=0.978, \Lambda=0.979, \Lambda=0.98, \Lambda=0.981, \Lambda=0.982, \Lambda=0.984, \Lambda=0.985, \Lambda=0.986, \Lambda=0.988, \Lambda=0.989$, $\Lambda=0.99, \Lambda=0.991, \Lambda=0.992, \Lambda=0.994, \Lambda=0.995, \Lambda=0.996, \Lambda=0.998, \Lambda=0.999$ and,$\Lambda=1.0$

a threshold point in applied voltage beyond which the fully coupled nonlinear solver becomes 
computational more favourable. Importantly, the staggered approach can also be applied to large displacements small strains problems. The staggered approach is competitive due to the fact that for its range of applicability, only the scalar field Gauss's law needs to be solved for iteratively.

Acknowledgement: The first author acknowledges the financial support received through the European Commission EACEA Agency, Framework Partnership Agreement 2013-0043 Erasmus Mundus Action 1b, as a part of the EM Joint Doctorate Simulation in Engineering and Entrepreneurship Development (SEED). The second author acknowledges the financial support received through the European Training Network AdMoRe.

\section{References}

[1] A. J. Gil, R. Ortigosa, A new framework for large strain electromechanics based on convex multivariable strain energies: Variational formulation and material characterisation, Computer Methods in Applied Mechanics and Engineering 302 (2016) 293 - 328.

[2] R. Ortigosa, A. J. Gil, A new framework for large strain electromechanics based on convex multivariable strain energies: Finite element discretisation and computational implementation, Computer Methods in Applied Mechanics and Engineering 302 (2016) 329 - 360.

[3] R. Ortigosa, A. J. Gil, A new framework for large strain electromechanics based on convex multi-variable strain energies: Conservation laws, hyperbolicity and extension to electro-magnetomechanics, Computer Methods in Applied Mechanics and Engineering 309 (2016) $202-242$.

[4] A. Erturk, D. J. Inman, Piezoelectric Energy Harvesting, first ed., John Wiley \& Sons Inc., Chichester, England, 2011.

[5] A. L. Dorfmann, R. W. Ogden, Nonlinear Theory of Electroelastic and Magnetoelastic Interactions, Springer, Dordrecht, Heidelberg, London, New York, 2014.

[6] S. R. Anton, H. A. Sodano, A review of power harvesting using piezoelectric materials (2003-2006), Smart Materials and Structures 16 (2007) R1.

[7] N. E. duToit, B. L. Wardle, S. G. Kim, Design considerations for MEMS-scale piezoelectric mechanical vibration energy harvesters, Integrated Ferroelectrics 16 (2005) 121-160.

[8] A. Benjeddou, Advances in piezoelectric finite element modeling of adaptive structural elements: a survey, Computers and Structures 76 (2000) 347-363.

[9] A. S. Henry, G. Park, J. I. Daniel, Estimation of electric charge output for piezoelectric energy harvesting, Strain 40 (2004) 49-58.

[10] N. D. Sharma, R. Maranganti, P. Sharma, On the possibility of piezoelectric nanocomposites without using piezoelectric materials, Journal of the Mechanics and Physics of Solids 55 (2007) 2328-2350.

[11] R. Maranganti, N. D. Sharma, P. Sharma, Electromechanical coupling in nonpiezoelectric materials due to nanoscale nonlocal size effects: Greens function solutions and embedded inclusions, Physical Review B 74 (2006) 014110.

[12] T. Dumitrica, C. M. Landis, B. I. Yakobson, Curvature-induced polarization in carbon nanoshells, Chemical Physics Letters 360 (2002) 182-188. 
[13] R. Poya, A. J. Gil, P. D. Ledger, A computational framework for the analysis of linear piezoelectric beams using $h p$-FEM, Computers and Structures 152 (2015) 155-172.

[14] D. K. Vu, P. Steinmann, Nonlinear electro- and magneto-elastostatics: Material and spatial settings, International Journal of Solids and Structures 44 (2007) 7891-7905.

[15] R. Bustamante, D. A., R. W. Ogden, Nonlinear electroelastostatics: a variational framework, Zeitschrift fur angewandte Mathematik und Physik 60 (2009) 154-177.

[16] A. J. Gil, R. Ortigosa, C. H. Lee, A computational framework for large strain nearly and truly incompressible electromechanics based on convex multi-variable strain energies, Computer Methods in Applied Mechanics and Engineering 310 (2016) 297 - 334.

[17] R. Ortigosa, A. J. Gil, J. Bonet, C. Hesch, A computational framework for polyconvex large strain elasticity for geometrically exact beam theory, Computational Mechanics 57 (2016) 277-303.

[18] R. Ortigosa, A. J. Gil, A computational framework for incompressible electromechanics based on convex multi-variable strain energies for geometrically exact shell theory, Computer Methods in Applied Mechanics and Engineering 317 (2017) 792 - 816.

[19] J. M. Ball, Convexity conditions and existence theorems in nonlinear elasticity, Archive for Rational Mechanics and Analysis 63 (1976) 337-403.

[20] J. E. Marsden, T. J. R. Hughes, Mathematical Foundations of Elasticity, Dover Civil and Mechanical Engineering, 1994.

[21] J. Schröder, P. Neff, V. Ebbing, Anisotropic polyconvex energies on the basis of crystallographic motivated structural tensors, Journal of the Mechanics and Physics of Solids 56 (2008) 3486 - 3506.

[22] J. Bonet, A. J. Gil, R. Ortigosa, On a tensor cross product based formulation of large strain solid mechanics, International Journal of Solids and Structures 84 (2016) 49-63.

[23] C. Miehe, D. Vallicotti, D. Zh, Computational structural and material stability analysis in finite electro-elasto-statics of electro-active materials, International Journal for Numerical Methods in Engineering 102 (2015) 1605-1637. Nme.4855.

[24] J.-S. Plante, S. Dubowsky, Large-scale failure modes of dielectric elastomer actuators, International Journal of Solids and Structures 43 (2006) 7727 - 7751.

[25] J. Zhou, W. Hong, X. Zhao, Z. Zhang, Z. Suo, Propagation of instability in dielectric elastomers, International Journal of Solids and Structures 45 (2008) 3739 - 3750. Special Issue Honoring K.C. Hwang.

[26] G. Mao, X. Huang, M. Diab, J. Liu, S. Qu, Controlling wrinkles on the surface of a dielectric elastomer balloon, Extreme Mechanics Letters 9 (2016) 139 - 146.

[27] J. Zhu, M. Kollosche, T. Lu, G. Kofod, Z. Suo, Two types of transitions to wrinkles in dielectric elastomers, Soft Matter 8 (2012) 8840-8846.

[28] E. Lejeune, A. Javili, C. Linder, An algorithmic approach to multi-layer wrinkling, Extreme Mechanics Letters 7 (2016) 10 - 17. Mechanics in Extreme Manufacturing.

[29] A. J. Gil, S. Adhikari, F. Scarpa, J. Bonet, The formation of wrinkles in single-layer graphene sheets under nanoindentation, Journal of Physics: Condensed Matter 22 (2010) 145302. 
[30] J. Mosler, A novel variational algorithmic formulation for wrinkling at finite strains based on energy minimization: Application to mesh adaption, Computer Methods in Applied Mechanics and Engineering 197 (2008) 1131 - 1146.

[31] J. Mosler, F. Cirak, A variational formulation for finite deformation wrinkling analysis of inelastic membranes, Computer Methods in Applied Mechanics and Engineering 198 (2009) 2087 - 2098.

[32] A. C. Eringen, G. A. Maugin, Electrodynamics of Continua I: Foundations and Solid Media, Springer, Dordrecht, Heidelberg, London, New York, 1990.

[33] L. D. Landau, E. M. Lifshitz, Electrodynamics of Continuous Media, Pergamon, Oxford, 1960.

[34] J. L. Pérez-Aparicio, R. Palma, R. L. Taylor, Multiphysics and thermodynamic formulations for equilibrium and non-equilibrium interactions: Non-linear finite elements applied to multi-coupled active materials, Archives of Computational Methods in Engineering (2015) 1-49.

[35] A. J. Gil, P. D. Ledger, A coupled $h p$-finite element scheme for the solution of two-dimensional electrostrictive materials, International Journal for Numerical Methods in Engineering 91 (2012) $1158-1183$.

[36] C. Rinaldi, H. Brenner, Body versus surface forces in continuum mechanics: is the maxwell stress tensor a physically objective cauchy stress?, Physical Review E 65 (2002) 036615.

[37] R. Bustamante, J. Merodio, On simple constitutive restrictions for transversely isotropic nonlinearly elastic materials and isotropic magneto-sensitive elastomers, Journal of Engineering Mathematics 68 (2009) 15-26.

[38] R. Poya, R. Sevilla, A. J. Gil, A unified approach for a posteriori high-order curved mesh generation using solid mechanics, Computational Mechanics 58 (2016) 457-490.

[39] J. Bonet, A. J. Gil, R. D. Wood, Nonlinear Solid Mechanics for Finite Element Analysis: Statics, 3rd ed., Cambridge University Press, Cambridge, UK, 2016.

[40] R. de Boer, Vektor- und Tensorrechnung für Ingenieure, Springer, 1982.

[41] J. Bonet, A. J. Gil, R. Ortigosa, A computational framework for polyconvex large strain elasticity, Computer Methods in Applied Mechanics and Engineering 283 (2015) 1061-1094.

[42] S. Bagwell, P. D. Ledger, A. J. Gil, M. Mallett, M. Kruip, A linearised $h p$-finite element framework for acousto- magneto-mechanical coupling in axisymmetric MRI scanners, International Journal for Numerical Methods in Engineering (2017) DOI: http://dx.doi.org/10.1002/nme.5559.

[43] Z. Q. Xie, R. Sevilla, O. Hassan, K. Morgan, The generation of arbitrary order curved meshes for 3D finite element analysis, Computational Mechanics 51 (2013) 361-374.

[44] R. Poya, A. J. Gil, R. Ortigosa, A high performance data parallel tensor contraction framework: Application to coupled electro-mechanics, Computer Physics Communications 216 (2017) 35 - 52.

[45] K. Stüben, A review of algebraic multigrid, Journal of Computational and Applied Mathematics 128 (2001) 281 - 309. Numerical Analysis 2000. Vol. VII: Partial Differential Equations.

[46] M. W. Gee, U. Küttler, W. A. Wall, Truly monolithic algebraic multigrid for fluid-structure interaction, International Journal for Numerical Methods in Engineering 85 (2011) 987-1016. 
[47] C. Danowski, V. Gravemeier, L. Yoshihara, W. A. Wall, A monolithic computational approach to thermo-structure interaction, International Journal for Numerical Methods in Engineering 95 (2013) 1053-1078.

[48] J. Šstek, F. Cirak, Parallel iterative solution of the incompressible Navier-Stokes equations with application to rotating wings, Computers \& Fluids 122 (2015) 165 - 183.

[49] R. Abgrall, C. Dobrzynski, A. Froehly, A method for computing curved meshes via the linear elasticity analogy, application to fluid dynamics problems, International Journal for Numerical Methods in Fluids 76 (2014) 246-266.

[50] A. Gargallo-Peiró, X. Roca, J. Peraire, J. Sarrate, Distortion and quality measures for validating and generating high-order tetrahedral meshes, Engineering with Computers (2014) 1-15.

[51] F. Witherden, P. Vincent, On the identification of symmetric quadrature rules for finite element methods, Computers \& Mathematics with Applications 69 (2015) 1232 - 1241.

[52] C. Johnson, Numerical Solution of Partial Differential Equations by the Finite Element Method, Dover Publications Inc., Mineola, New York, USA, 2009.

[53] P. Solin, K. Segeth, Higher-Order Finite Element Methods, Chapman \& Hall, 2003.

[54] J. Schröder, D. Gross, Invariant formulation of the electromechanical enthalpy function of transversely isotropic piezoelectric materials, Archive of Applied Mechanics 73 (2004) 533-552.

[55] D. K. Vu, P. Steinmann, G. Possart, Numerical modelling of non-linear electroelasticity, International Journal for Numerical Methods in Engineering 70 (2007) 685-704.

[56] J.-P. Pelteret, D. Davydov, A. McBride, D. K. Vu, P. Steinmann, Computational electro-elasticity and magneto-elasticity for quasi-incompressible media immersed in free space, International Journal for Numerical Methods in Engineering (2016) DOI: http://dx.doi.org/10.1002/nme.5254.

[57] R. Sevilla, S. Fernández-Méndez, A. Huerta, Comparison of high-order curved finite elements, Internat. J. Numer. Methods Engrg. 87 (2011) 719-734.

[58] R. Sevilla, S. Fernández-Méndez, A. Huerta, NURBS-Enhanced Finite Element Method (NEFEM): a seamless bridge between CAD and FEM, Archives of Computational Methods in Engineering 18 (2011) 441-484.

[59] E. G. Thompson, M. I. Haque, A high order finite element for completely incompressible creeping flow, International Journal for Numerical Methods in Engineering 6 (1973) 315-321.

[60] V. John, G. Matthies, Higher-order finite element discretizations in a benchmark problem for incompressible flows, International Journal for Numerical Methods in Fluids 37 (2001) 885-903.

[61] G. Wells, L. Sluys, R. de Borst, A p-adaptive scheme for overcoming volumetric locking during plastic flow, Computer Methods in Applied Mechanics and Engineering 191 (2002) 3153 - 3164.

[62] J. Schröder, P. Wriggers, D. Balzani, A new mixed finite element based on different approximations of the minors of deformation tensors, Computer Methods in Applied Mechanics and Engineering 200 (2011) $3583-3600$. 
[63] C. Meier, A. Popp, W. A. Wall, A locking-free finite element formulation and reduced models for geometrically exact Kirchhoff rods, Computer Methods in Applied Mechanics and Engineering 290 (2015) $314-341$.

[64] C. Meier, A. Popp, W. A. Wall, An objective 3D large deformation finite element formulation for geometrically exact curved Kirchhoff rods, Computer Methods in Applied Mechanics and Engineering 278 (2014) $445-478$.

[65] K. Bertoldi, M. Gei, Instabilities in multilayered soft dielectrics, Journal of the Mechanics and Physics of Solids 59 (2011) $18-42$.

[66] de Souza Neto E. A., D. Perić, D. R. J. Owen, Computational Methods for Plasticity: Theory and Applications, first ed., John Wiley \& Sons, Chichester, West Sussex, United Kingdom, 2008.

[67] M. A. Crisfield, Nonlinear Finite Element Analysis of Solids and Structures: Essentials, John Wiley \& Sons, Chichester, West Sussex, United Kingdom, 1996.

[68] R. de Borst, M. A. Crisfield, J. J. C. Remmers, C. V. Verhoosel, Nonlinear Finite Element Analysis of Solids and Structures, second ed., John Wiley \& Sons, Chichester, West Sussex, United Kingdom, 2012 . 